Claremont Colleges

Scholarship@ Claremont

CGU Theses \& Dissertations

CGU Student Scholarship

2012

\title{
A Fire Simulation Model for Heterogeneous Environments Using the Level Set Method
}

Shin-en Lo

Claremont Graduate University

\section{Recommended Citation}

Lo, Shin-en, "A Fire Simulation Model for Heterogeneous Environments Using the Level Set Method" (2012). CGU Theses \& Dissertations. Paper 72.

http://scholarship.claremont.edu/cgu_etd/72

DOI: $10.5642 /$ cguetd $/ 72$

This Open Access Dissertation is brought to you for free and open access by the CGU Student Scholarship at Scholarship @ Claremont. It has been accepted for inclusion in CGU Theses \& Dissertations by an authorized administrator of Scholarship @ Claremont. For more information, please contactscholarship@cuc.claremont.edu. 


\section{A Fire Simulation Model for Heterogeneous Environments Using the Level Set Method}
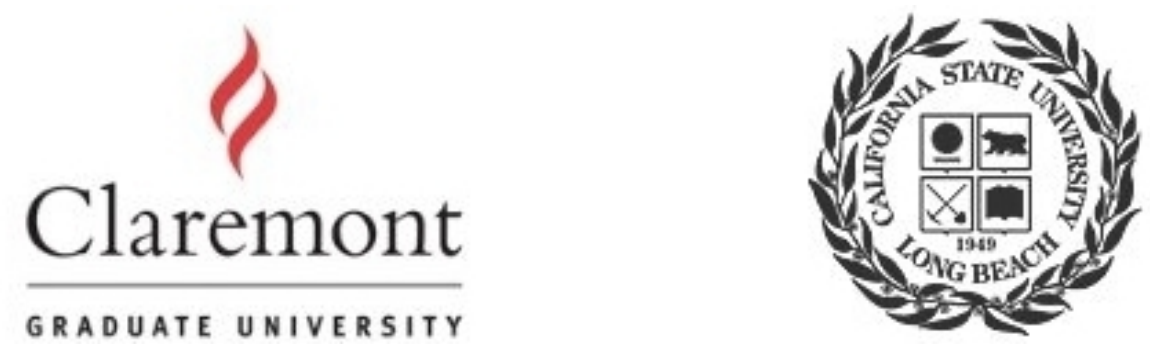

Shin-en Lo

Institute of Mathematical Sciences

College of Engineering

Claremont Graduate University

California State University, Long Beach

A thesis submitted to the Faculty of Claremont Graduate University and California State University, Long Beach for the degree of

Doctor of Philosophy in Engineering and Industrial Applied Mathematics 


\section{APPROVAL OF THE COMMITTEE}

We, the undersigned, certify that we have read, reviewed, and critiqued the thesis of Shin-en Lo and do hereby approve it as adequate in scope and quality for meriting the degree of Doctor of Philosophy in Engineering and Industrial Applied Mathematics.

CGU Committee:

Dr. Ellis Cumberbatch

Dr. Ali Nadim
CSULB Committee:

Dr. Eun Heui Kim

Dr. Chung-min Lee

Committee Chair

Dr. Burkhard Englert

Date Approved: 


\section{Abstract \\ A Fire Simulation Model for Heterogeneous Environments Using the Level Set Method \\ by \\ Shin-en Lo \\ Claremont Graduate University: August 2012}

Wildfire hazard and its destructive consequences have become a growing issue around the world especially in the context of global warming. An effective and efficient fire simulation model will make it possible to predict the fire spread and assist firefighters in the process of controlling the damage and containing the fire area. Simulating wildfire spread remains challenging due to the complexity of fire behaviors. The raster-based method and the vector-based method are two major approaches that allow one to perform computerized fire spread simulation. In this thesis, we present a scheme we have developed that utilizes a level set method to build a fire spread simulation model. The scheme applies the strengths and overcomes some of the shortcomings of the two major types of simulation method. We store fire data and local rules at cells. Instead of calculating which are the next ignition points cell by cell, we apply Huygens' principle and elliptical spread assumption to calculate the direction and distance of the expanding fire by the level set method. The advantage to storing data at cells is that it makes our simulation model more suitable for heterogeneous fuel and complex topographic environment. Using a level set method for our simulation model makes it possible to overcome the crossover problem. Another strength of the level set method is its continuous data processing. Applying the level set method in the simulation models, we need fewer vector points than raster cells to produce a more realistic fire shape. We demonstrate this fire simulation model through two implementations using narrow band level set method and fast marching method. The simulated results are compared to the real fire image data generated from Troy and Colina fires. The simulation data are then studied and compared. The ultimate goal is to apply this simulation model to the broader picture to better predict different types of fires such as crown fire, spotting fires, etc. 


\section{Acknowledgements}

The author would like to thank the following people:

Dr. Englert, for his insight, advise and continuous encouragement throughout this process.

Dr. Ellis Cumberbatch, Dr. Eun Heui Kim, Dr. Chung-Min Lee, Dr. Ali Nadim, for their knowledge, edits, comments and support.

Dr. Francis M. Fujioka, for his advice and expertise on wild-land fire behavior modeling.

I want to show my gratitude to late Professor Hedley Morris who guided me at the initial phase of my research. I am benefited from his kindness and wisdom.

Last but not least, my thanks go to my parents for always having faith on me; for my wife, Maggie and my son, Stephen whose endless love and support always bless me. 


\section{Contents}

$\begin{array}{lll}\text { List of Figures } & \text { ix }\end{array}$

List of Tables

Glossary Xiii

1 Introduction 1

1.1 Introduction . . . . . . . . . . . . . . . . . . 1

1.2 Goals and Objectives of the Research . . . . . . . . . . . . 5

1.3 Organization of this Thesis $\ldots \ldots \ldots \ldots$

2 Wildfire Models

2.1 History . . . . . . . . . . . . . . . . . . . . . 7

2.2 Wildfire Models . . . . . . . . . . . . . . . . 8

2.2.1 Theoretical (Physics Based) Models . . . . . . . . . . . 8

2.2 .2 Empirical Methods . . . . . . . . . . . . . . . . . . 9

2.2.3 Semi-Empirical Methods . . . . . . . . . . . . . . . . . . . . 10

2.2.3.1 The Vector Method . . . . . . . . . . . . . 12

2.2.3.2 Vector-Based Simulation Approach . . . . . . . . . 14

3 Methodologies 17

3.1 Strategy and Solution . . . . . . . . . . . . . . . 17

3.1 .1 Strategy . . . . . . . . . . . . . . . . 18

3.1.2 Modification of Rothermel's Model . . . . . . . . . . . . . 19

3.2 The Level Set Method . . . . . . . . . . . . . . . . . . . . . . 22

3.2.1 The Narrow Band Method . . . . . . . . . . . . . . . . 26

3.2.1.1 Numerical Solution for Narrow Band Method . . . . . . 26 
3.2.1.2 The Algorithm of Narrow Band Method . . . . . . . 28

3.2.2 Strategy for Wildfire Simulation Using the Narrow Band Level

Set Method . . . . . . . . . . . . . . . . 29

3.2 .3 Methodology Steps . . . . . . . . . . . . . . . . . . 30

3.3 Fast Marching Method . . . . . . . . . . . . . . . . . 30

3.3.1 Numerical Solution for Fast Marching . . . . . . . . . . . . . . 34

3.3.2 Fast Marching Methodology Steps . . . . . . . . . . . . . 35

3.4 Simulation Model . . . . . . . . . . . . . . . . . . . . . . 37

4 Simulation and Numerical Analysis 41

4.1 Simulation Data and Equation . . . . . . . . . . . . . . 41

4.2 Homogeneous Landscapes . . . . . . . . . . . . . . . . . . 44

4.2 .1 Case 1: No wind and zero slope . . . . . . . . . . . . 44

4.2 .2 Case 2: Wind only . . . . . . . . . . . . . . . . . 44

4.2 .3 Case 3: Under wind and with slope . . . . . . . . . . . 45

4.3 Heterogeneous Landscapes . . . . . . . . . . . . . . . . . . . . . . . 51

4.3.1 Case 1: Two types of fuel . . . . . . . . . . . . 51

4.3 .2 Case 2: Fuel only . . . . . . . . . . . . . . . 5 54

4.3.3 Case 3: Complicated terrain and fuel type with wind . . . . . 554

4.4 Numerical Accuracy Analysis . . . . . . . . . . . . . . . . . . . 57

4.4.1 Verification Conclusion . . . . . . . . . . . . . 60

5 Case Studies 63

5.1 Case I : Troy Fire . . . . . . . . . . . . . . . . . . . 65 65

5.1.1 Narrow Band Level Set Method . . . . . . . . . . . . . . 66 66

5.1 .2 Fast Marching Method . . . . . . . . . . . . . . . 68 68

5.2 Case II : Colina Fire . . . . . . . . . . . . . . . . . . . . . . . . 71

5.2.1 Narrow Band Level Set Method . . . . . . . . . . . . . . . 774

5.2 .2 Fast Marching Method . . . . . . . . . . . . . . . 775

5.3 Numerical Result Analysis . . . . . . . . . . . . . . . . . . . . . . 78

6 Conclusion 81

Bibliography 85 


\section{List of Figures}

1.1 Two major computer fire simulation methods . . . . . . . . . . 2

1.2 Huygens' principle . . . . . . . . . . . . . . . . . . . 3

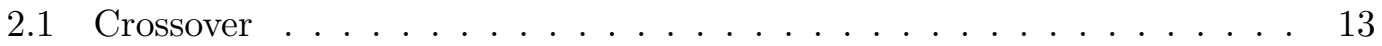

2.2 An Elliptical Shape Fire-spread Assumption . . . . . . . . . . . . 15

3.1 Wind coefficient for ROS (dimensionless) . . . . . . . . . . . 21

3.2 Evolution of a distance function . . . . . . . . . . . . . . . . 24

3.3 Narrow band surface . . . . . . . . . . . . . . . . . . . . . . 32

3.4 Fast Marching Algorithm . . . . . . . . . . . . . . . . . 33

3.5 Model structure . . . . . . . . . . . . . . . . . . . . 37

3.6 Sample results from the model $\ldots \ldots \ldots$. . . . . . . . . . . . .

4.1 A basic fire simulated using two different methods . . . . . . . . . 45 45

4.2 A fire with west wind simulated using two different methods . . . . . . 4 46

4.3 Surface fire shapes generated by the narrow band level set method . . . 47

4.4 Surface fire shapes generated from the fast marching method . . . . . . 49

4.5 Fire passes a hill . . . . . . . . . . . . . . . . . 50 50

4.6 Slope $\tan \vartheta_{t}$ and fire front $\ldots \ldots \ldots \ldots$

4.7 Fire passes a depression under west wind . . . . . . . . . 51

4.8 A fire expand at Heterogeneous fuel . . . . . . . . . . . . . . . 5 53

4.9 A fire with Heterogeneous fuel . . . . . . . . . . . . . . . . 55

4.10 A fire simulated in a natural environment . . . . . . . . . . . 56

4.11 Error analysis of a basic fire . . . . . . . . . . . . . 5 58

4.12 Execution time at different resolutions . . . . . . . . . . . . 60 60

4.13 Different resolution maps of Troy . . . . . . . . . . . . . . . 62 62 
5.113 FBFM color codes . . . . . . . . . . . . . . . . . . 63 63

5.2 Map of Troy . . . . . . . . . . . . . . . 65 65

5.3 Troy fire and Time table . . . . . . . . . . . . 66 66

5.4 Terrain and vegetation maps of Troy . . . . . . . . . . . 67 67

5.5 Moisture data table for fuel models . . . . . . . . . . . 67 67

5.6 Simulated Troy fire . . . . . . . . . . . . . . . . . 69 69

5.7 Troy fire comparison measure . . . . . . . . . . . . . . 70

5.8 Comparison of the final photo to the simulation . . . . . . . . . 70

5.9 Troy fire simulation using the fast marching method . . . . . . . . 71

5.10 Troy fire simulation comparison using the fast marching method . . . 72

5.11 Map of Colina . . . . . . . . . . . . . . . . 72

5.12 Colina fire and weather data . . . . . . . . . . . 73

5.13 Terrain and vegetation maps of Colina . . . . . . . . . . 74

5.14 Colina fire simulated using narrow band level set method . . . . . . 76

5.15 Fire simulated using narrow band method compared to actual Colina fire 77

5.16 Colina fire simulation produced from the fast marching method . . . . 78 


\section{List of Tables}

3.1 Level set pseudo-code . . . . . . . . . . . . . . . . . . . 31

3.2 Fast marching pseudo-code . . . . . . . . . . . 36

4.1 Fire simulation parameters I . . . . . . . . . . . . . . . 42

4.2 Standard 13 fuel models . . . . . . . . . . . . . . . . . 43

4.3 Fuel model parameters . . . . . . . . . . . . . . . . . 44

4.4 Fire simulation parameters II . . . . . . . . . . . . . . . 45

4.5 Execution time for a basic fire . . . . . . . . . . . . 5 58

4.6 Error analysis of a basic fire . . . . . . . . . . . . 5 58

4.7 Resolution $\triangle x$ analysis . . . . . . . . . . . . . . . . 59

4.8 Execution time at different resolutions . . . . . . . . . . . . 60

5.1 Parameters of fuel model for case study . . . . . . . . . . . . 64 64

5.2 Comparison of simulated Colina fire using narrow band level set method 75

5.3 Case study analysis . . . . . . . . . . . . . . . . . . . . 79 79 


\section{Glossary}

$\begin{array}{ll}\Phi_{s} & \text { Terrain coefficient } \\ \Phi_{w} & \text { Wind coefficient } \\ \rho_{b} & \text { Over-dry bulk density }\left(\mathrm{kg} / \mathrm{m}^{3}\right) \\ \theta & \begin{array}{l}\text { The angle between head fire and } \\ \text { wind direction (radians) }\end{array} \\ \theta_{t} & \text { The slope angle of terrain (radians) } \\ \varepsilon & \text { Effective heating number } \\ C I & \text { Combustibility index }\end{array}$

$\begin{array}{ll}I_{R} \xi & \text { The propagating flux ratio } \\ I_{R} & \text { Reaction intensity }\left(\mathrm{kJ} / \mathrm{min} \cdot \mathrm{m}^{2}\right) \\ Q_{i g} & \text { Heat of ignition }(\mathrm{kJ} / \mathrm{kg}) \\ R & \text { Heading fire spread rate }(\mathrm{m} / \mathrm{min})\end{array}$

13FBFM 13 fire behavior fuel models which are taken from Anderson (1983) 2

53FBFM 53 standard fire behavior fuel models which is originating from the 13FBFM and further expanded to forty additional fuel models by Scott and Burgan 29 .

DEM Digital elevation model

FMC Fuel moisture content

GIS Geographical information systems

ROS The rate of forward spread of the head fire 


\section{1}

\section{Introduction}

\subsection{Introduction}

Wildfires continue to present a hazard that adversely impacts public safety and public health, resulting in a significant negative economic impact on individuals, insurance companies and society as a whole. According to the statistics published by the U.S. Fire Administration [4], a total of 406,614 wildfires occurred that burned 39,105,433 acres during the five-year period from 2005-2009 in the United States. The fires caused 3, 010 deaths and 17,050 injuries with a direct dollar loss in 2009 alone of over $\$ 12$ billion. Wildfires cause monetary damage and environmental damage every year. However, wildfire spread continues to be rather unpredictable. There is a growing demand to come up with faster and more reliable ways to predict the spread of wildfires. Developing more predictive simulation models will help fire safety managers in their fire suppression and containment efforts to reduce life and monetary losses.

Several computer simulation models have been developed to simulate fire spread. For the most part, they are based on one of two major methods: the raster-based cellular discrete method (Fig. 1.1a) and the vector-based polygon expanding method (Fig. 1.1b] 23] [34] 33].

The raster-based method simulates fire growth as a discrete process of ignitions across a regularly spaced landscape grid referred to as a cellular grid, similar to cellular automata $(\mathrm{CA})$ which is based on the contiguous principle, a cell may be ignited by 


\section{INTRODUCTION}

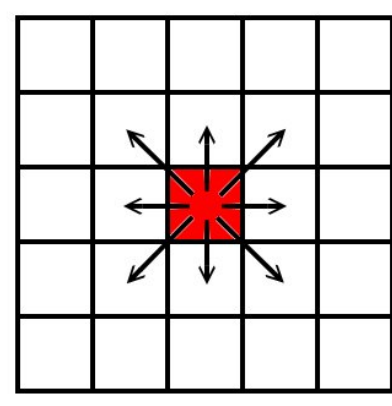

(a) Raster-based method, which is based on the Contiguous principle, simulates fire spread.

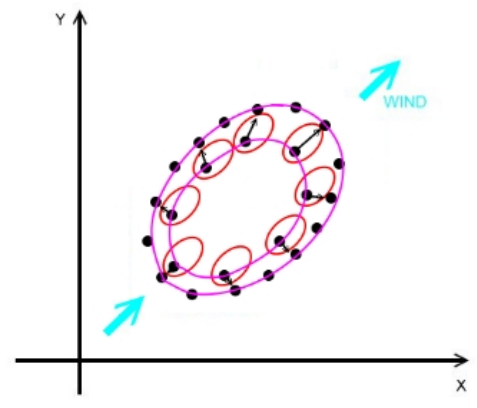

(b) Vector-based method, which is based on the Huygens' principle \& the elliptical shape assumption, simulates fire expanding.

Figure 1.1: Two major computer fire simulation methods - The two most widely used fire simulation methods in softwares are the raster-based cellular discrete method and the vector-based polygon expanding method.

its burned neighboring cells depending on certain conditional levels such as fuel, wind, etc. The fundamental properties of these methods are the definition of the state of a cell that is called state function and the local rules that update the state on sequential time intervals. Storing data at cells is more suitable for heterogeneous fuel and complex topographic environment. However, there is no way to determine the next movement of the fire front on the grid; raster-based method requires further calculations on the neighboring cells of the specific cell in order to determine the fastest rate of fire spread (ROS) or the shortest travel time to the next neighboring cell most likely to be ignited. Furthermore, in the raster-based method, the fire spread processing is not a continuous process. The resolution is critical for the output of the simulation. Higher resolution produces better fire shape at the price of consuming bigger processing memories. The raster-based method falls short in delivering high resolution fire shape.

Vector-based methods apply fire spread formula to calculate the traveling direction and the distance of each vertex on the fire polygon to move the vertex forward and expand the fire. They are built upon two principles. The first is Huygens' principle which assumes that the fire front propagates from the vertex on the front 
edge equally and independently. An illustration of this idea is shown at Fig. 1.2. The second is to assume that the fire spread shape will appear to be elliptical under the wind. Fire polygon function is defined by a series of two dimensional vertices which point to $x y$ coordinates. The expanding distance of a fire polygon function is determined by computing the parameters such as wind, fuel type and terrain from each vertex to get the spread rate and multiplied by the time step. It is a continuous data process that requires fewer vector points than

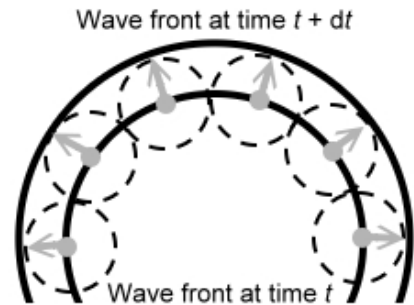

Figure 1.2: Huygens' principle Huygens' principle assumes that the wave front propagates from the vertex on the front edge equally and independently. raster cells to produce more realistic fire shapes while requiring less computer memory. The most difficult task for the vector based methods is to calculate the crossover which refers to the overlap of two fire fronts. It is definitely a tedious, time-and resource-consuming process to calculate the crossover [9]. Another limitation of the vector method is the omission of the ignition point between different fuel types. The result is that the fire spread simulation using vector based methods will assume a nonstop process until it meets water or rock. In the real world, the heat from a fuel may or may not be sufficiently intense to spread to another type of fuel.

In this thesis we developed a scheme that utilizes the distance function of a level set method to build a fire spread simulation model. The scheme leverages the strength of the two major computer simulation methods and at the same time overcomes the limitations of these methods. The main idea is to apply the level set method to heterogeneous environments. First, we stored local data at each cell. Then by applying a distance function on the plane, we transformed the distance numbers into a 3D object and changed its contour forward or backward by moving this 3D object. Lastly, we applied PDE/ODE mathematical methods (level set function) combining the data from raster cells to calculate the contour's expanding distance on the $x y$-plane to predict the fire spread. 


\section{INTRODUCTION}

Two mathematical methods conform to our proposed scheme - the narrow band method and the fast marching method which deviated from the level set concept. Both view $2 \mathrm{D}$ surface evolution from three dimensions. The level set method is a numerical tool introduced by Osher and Sethian [22] and is popular for tracking and simulating the dynamics of moving surfaces. It transfers the problem from $x y$-plane to a higher dimension, then projects the problem back to the $x y$-plane in order to handle propagating contours. It is well known for its ability to easily handle topological changes such as merging or breaking interfaces. Therefore the level set method can be applied to resolve the crossover issue in fire spread simulation using vector-based methods.

The fast marching method introduced by Sethian [30 also views 2D expansion from a $3 \mathrm{D}$ perspective. If the contour from the $3 \mathrm{D}$ object is always either expanding or shrinking, it provides a stationary formulation on a discrete grid where the contour crosses each grid no more than once. Instead of calculating the travel distance in a level set method, the fast marching method calculates the travel time from points to the surface to track the moving surface.

By using the narrow band method, a fire spread simulation model was created to verify our scheme. First, we built a $3 \mathrm{D}$ level set function $\phi(x(t), y(t), t)$ from a distance function and used the data which had been pre-stored at each cell to calculate the fire forward distance by fire formula. We used the Hamilton-Jacobi method to solve the level set equation $\phi_{t}=-F\|\nabla \phi\|$ and to estimate the contour evolution on the $x y$ plane.

For the fast marching method, we used the pre-stored data at each cell to calculate the travel time from fire front by solving the Eikonal equation $1=F\|\nabla T\|$ to build a time matrix. Then we transformed this matrix to a $3 \mathrm{D}$ object $T(x, y, t)$ and used its projections on the $x y$-plane to simulate the fire spread.

The fire formula used in our model to calculate the fire spread speed $F$ is Rothermels fire model [28] which is the most popular mathematical fire model used in the United States and delivers a good estimate of the fire spread. We modified Rothermel's fire model and used it to simulate the fire spread in the narrow band level set method, 
and fast marching method.

\subsection{Goals and Objectives of the Research}

As discussed previously, a wildfire is a complex multi-scale process affected by non-linear scale-dependent interactions with other earth surface processes. A lot of research and several methods are devoted to simulate the spread of a fire front. This research leveraged the raster concept to divide the landscape into identical square cells and to store pre-exiting data at cells, then applying the level set method to evolve fire surface for more complex fire environments.

The goal of this research is to overcome some of the shortcomings of the two computer simulation methods. We store fire data and local rules at cells, instead of calculating which are the next ignition points cell by cell, then we apply Huygens' principle and elliptical spread assumption to calculate the direction and distance to expand fire. The vector-based method using a polygon equation to simulate the fire front spread speed does not save any information, resulting in two major shortcomings. First is the crossover issue and the second is the inability to replicate the natural wildfire spread between two different types of fuel. For our simulation model, storing data at cells makes it suitable for heterogeneous fuel in a complex topographic environment. Using the level set method for our simulation model overcomes the crossover problem.

The ultimate goal is to apply this simulation model to the broad scope to better predict different types of fires such as crown fire, spotting fire, etc. Further, the goal is to generate an improved simulation model and apply it to other scenarios such as smog pollution, oil spill and volcanic ash spread.

The more limited scope of this research is to consider only surface fires where the fire front spreads in a layer just above the ground. Crown fires, ground fires, and spotting fires will be considered in future studies. 


\section{INTRODUCTION}

\subsection{Organization of this Thesis}

This thesis is organized as follows:

Chapter 1 provides the problem statement for fire spread prediction in the introduction and states the goals and objectives of this research.

Chapter 2 describes the history and theories of wild fire research; categories of fire models; theories of the major simulation methods for fire spread and their strengths and limitations and presents the scheme used in our simulation model.

Chapter 3 describes the concept of building our fire simulation model; the mathematical theories, implementations of the level set methods which includes narrow band method and fast marching method; numerical methods for our model to simulate the fire spread; and, finally, presents the structure of our fire simulation model.

Chapter 4 applies the fire simulation model built from previous chapters to simulate the fire spread on hypothetical landscapes under various scenarios of fuel, wind and topography. The last part presents a numerical accuracy analysis.

Chapter 5 contains case studies. We use the data from the Pacific Southwest (PSW) Research Station to simulate real fire events that occurred in the Troy area in 2002 and Colina area in 2007. We compare the results between the implementation using narrow band level set method and the implementation using the fast marching method.

Chapter 6 summarizes the performance of this improved fire simulation model and makes recommendations for future studies. 


\section{2}

\section{Wildfire Models}

In this chapter, we discuss the history of research on wildfire models. Wildfire models can be divided into three categories: theoretical, empirical, and semi-empirical. They all focus on the development of a mathematical fire spread formula to predict the rate of spread of the head fire. One of the most commonly used methods is a semi-empirical method from Rothermel[28]. We modify Rothermel's model and use it as the fire spread formula in our fire simulation model. The mathematical theories that support the mathematical fire spread models are discussed. Those theories will be applied to our simulation model.

\subsection{History}

Wildfire modeling research originated in the 1920s. The earliest research is recorded in the works of Hawley(1926) [14], and Gisborne(1927, 1929) [12] [13] who tried to understand the wildfire phenomenon. They considered the relevant elements of fire by observing and measuring forest fires. Later they developed a framework for understanding wildfire behavior that enabled them to construct operational models of fire behavior to predict fire spread. From the early 1940s, researchers focused either on how to build mathematical fire spread models based on the fundamentals of the physical and chemical theories or on empirical methods. In 1946, Wallace R. Fons [10] brought forward a rigorous physical approach to model fire behavior using mathematical methods. Fons might be the first person trying to use a mathematical model to describe 


\section{WILDFIRE MODELS}

fire spread [28]. In the 1950s, formal research began by various government agencies such as the Commonwealth Forestry and Timber Bureau in Australia; Canadian Forest Service in Canada; and Forest Service in the USA. As the result of an increased budget and national interest, the research transitioned from understanding and controlling fire spread to the analysis and simulation of large forest or conflagration fires. After the 1990s, a rapid development in the capabilities of remote sensing, geographical information systems(GIS) and computing power made it possible to create simulation models to predict wildfire spread across a landscape.

\subsection{Wildfire Models}

Wildfire spread model research is attempting to create mathematical fire spread models. The research focuses on the rate of wildfire spread. Paster et al. [23] proposes classifying mathematical models into theoretical, empirical, and semi-empirical models depending on whether the model is based on purely physical understanding, statistical observation, or a combination of both.

\subsubsection{Theoretical (Physics Based) Models}

Theoretical models are generated from physical laws such as the laws of fluid mechanics, combustion and heat transfer. The fire spread is the result of the combination of chemical and physical processes. The combustion formula is:

Fuels + Oxygen + Energy (heat to ignition) $\longrightarrow$ Carbon dioxide + Water + Energy(heat) By the law of conservation, total mass never changes in the combustion process.

Many physics-based models are based on the above basic physics principles. It is desirable, perhaps suitable to use physics based models for wildfire spread. However, due to the nature of the wildfire spread, it will require further information beyond the basic physics principles. Most physics based models alone will not be able to forecast the wildfire spread in the real world. As Sullivan [34] pointed out "the primary use of such models is the study of fires under conditions, fuels and topographies that are not 
amenable to field experimentation."

\subsubsection{Empirical Methods}

Empirical methods combine statistical correlations from observation and experiments or real historical wildfire studies. The empirical models determine the key characteristics used to describe the behavior of the fire. The models focus mainly on the fire head, the rate of forward spread (ROS) of the fire head, the height of the flames, and the angle of the flames. The primary use of such models is to estimate where the spread is heading in the midst of the wind which is crucial information for firefighters in order that they can develop a fire extinguishing plan. Therefore empirical models have traditionally been one dimensional models that predicted the ROS of the fire head in the direction of the wind. Empirical models are most commonly used for operational fire simulations in use today.

Two of the main observations supporting empirical models are the analysis of fire spread based on different fuels and the measurement of head fire speed and direction based on wind speed and slope. Two general types of equations can be used to assess the dependency of fire spread rate $(V)$ on wind speed $(U)$ :

Exponential equation (Noble er al.(1980)[21]):

$$
V=V_{0} * \exp (a U)
$$

and

Power law equation (Thomas(1971) [35]):

$$
V=V_{0}+b * U^{c}
$$

where $V_{0}$ is the rate of spread in the absence of wind and $\mathrm{a}, \mathrm{b}$, and $\mathrm{c}$ are parameters to control the magnitude of fire on different fuels. For example, McArthur developed a fire danger meter $V=V_{0} \cdot \exp \left(0.145 U_{10}\right)$ and $V=V_{0} \cdot \exp \left(0.0842 U_{10}\right)$ for Australia grasslands and Australia forests respectively, where $U_{10}$ is the $10 \mathrm{~m} / \mathrm{s}$ wind speed [21].

There are two basic equations that model the impact of the slope on the fire spread,

$$
V=V_{0}+a \cdot(\tan \vartheta)^{c}
$$




\section{WILDFIRE MODELS}

and

$$
V=V_{0} * \exp (b \vartheta)
$$

Many wildfire spread models are based on those basic ideas and add some special parameters acquired from observations.

\subsubsection{Semi-Empirical Methods}

Semi-empirical models are a combination of a theoretical and empirical models. They are composed of simple, general physical theories, and completed through experimentation. The most famous Semi-empirical model is Rothermel's, [28], which was published in 1972 and is the first model to simulate wildfires. It was developed from Frandsen's theory [11] which used the conservation of energy ahead of an advancing fire and assumed that the fire occurred under a homogeneous fuel bed. This model focuses on the energy transfer between neighboring fuel cells as they ignite assuming uniform fuel at the surface which burns at a height of less than two meters from the ground.

The rate of spread (ROS) in Rothermel's model is the ratio of the propagating heat flux to the energy required to dry and preheat unburned fuels until they ignite. The framework defines the heat source as a reaction intensity $I_{R}$, which is the expression of fuel load, fuel particle size, fuel chemistry, fuel arrangement, and fuel moisture. Propagating flux combines the effect of forward radiation, convection(including flame contact), and piloted ignition. It is the product of $I_{R}$ and the propagating flux ratio, $\xi$, the latter term representing the proportion of reaction intensity that is transferred to the unburned fuels.

Rothermel's model assumes that the spread rate is based on an energy balance ratio of power source and heat sink terms:

$$
R=\frac{\text { Power density of propagating flux }}{\text { Preheating energy of fuel }}=\frac{I_{p}}{\rho_{b} Q_{T}}
$$

where $I_{p}$ is the fire propagating intensity provided by the heat source, $Q_{T}$ is the heat sink energy required to heat, dry, and pyrolyze the fuel, and $\rho_{b}$ is the fuel bed bulk density. 
As a mathematical expression, in Rothermel's model, the wildfire spread rate is given by:

$$
R=\frac{I_{R} \xi\left(1+\Phi_{w}+\Phi_{s}\right)}{\rho_{b} \varepsilon Q_{i g}}
$$

- $R$ : head fire spread rate $(\mathrm{m} / \mathrm{min})$

- $I_{R}$ : reaction intensity $\left(\mathrm{kJ} / \mathrm{min} \cdot \mathrm{m}^{2}\right)$

- $\xi$ : the propagating flux ratio

- $\rho_{b}$ : over-dry bulk density $\left(\mathrm{kg} / \mathrm{m}^{3}\right)$

- $\varepsilon$ : effective heating number

- $Q_{i g}$ : heat of ignition $(k J / k g)$

- $\Phi_{w}$ : wind coefficient

- $\Phi_{s}$ : slope factor

Reaction intensity $I_{R}$ :

The reaction intensity $I_{R}$ is the rate at which burning grasses release energy. The unit $\mathrm{kJ}$ is 'kilojoule'.

The propagating flux ratio $\xi$ :

This is the basic flux component with no wind $\left(\Phi_{w}\right)$ or slope $\left(\Phi_{s}\right)$. The Rothermel model assumes that the reaction intensity $I_{R}$ is independent and can be correlated with the propagating flux such that $I_{R} \xi=f\left(I_{R}\right)$.

Over-dry bulk density $\rho_{b}$ :

This fuel density combines with other parameters to give $\rho_{b}=w_{o} / \delta$ where $w_{o}$ is the over-dry fuel loading and $\delta$ is the fuel depth.

Effective Heating Number $\varepsilon$ :

$\varepsilon$ is the ratio of the effective bulk density $\rho_{b} \varepsilon$ to the actual bulk density $\rho_{b}$. $\varepsilon$ is always less than one and approaches zero as the fuel size increases. 


\section{WILDFIRE MODELS}

Heat of ignition $Q_{i g}$ :

$Q_{i g}$ describes the heat required to ignite fuel. It depends on:

- ignition temperature

- the ratio of fuel moisture

- fuel size, that is the amount of fuel available

Wind $\Phi_{w}$ :

$$
\Phi_{w}=C(3.28 U)^{B}\left(\frac{\beta}{\beta_{o p}}\right)^{-E}
$$

where $C, B$ and $E$ are functions of the fuel particle sizes in the fuel bed and $\beta$ is the packing ratio of the fuel bed.

Terrain $\Phi_{s}$ :

$$
\Phi_{s}=5.275 \beta^{-0.3}\left(\tan \vartheta_{t}\right)^{2}
$$

where $\tan \vartheta_{t}$ is the slope and $\beta$ is the packing ratio of the fuel bed.

Rothermel's model is the most popular fire model used in the U.S. Further details on Eq. 2.5 may be found in [28. It computes the steady-state fire spread rate in one-dimension parallel to the ground surface. Since Rothermel's model only predicts a one-dimensional fire spread in the heading direction, the assumption of an elliptic fire shape is crucial. Based on this assumption, we are able to calculate the flank and back fire with wind in this study.

\subsubsection{The Vector Method}

The vector-based method, also called polygon expanding method, is one of the major fire simulation methods where the fire front is defined by two-dimensional vertices on the $x y$ coordinates. The fire growth depends on the spread rate and direction of each vertex. The number of vertices increases as fire expands over time as shown in Fig. 1.1b on page 2, The vector method is based on two assumptions. The first assumption is Huygens' principle which states that each vertex on the fire-front is independent of the others and expands in the same way from the previous point. Based on this assumption, 
the fire growth is calculated on the fire front and is propagated at the fire front edge to an unburned area. This assumption requires information such as time, direction, and the rate of fire spread for the points on the fire front edge. The second assumption is that the fire shape is an ellipse under the wind. With these two assumptions, we can now successfully apply the one-dimensional fire spread model to the fire front points to get the rate of fire spread on the fire head and calculate the direction for the fire expansion to form the fire shape. Simulation models based on the vector method can produce a much more realistic fire perimeter [15] 33.

The wildfire may possibly have overlaps, called crossovers, which present a big challenge for the vector-based method. Most of the polygon fire-spread functions can be created from the two formulas Eq. (2.1) and Eq. (2.2) described earlier. But polygon functions do not distinguish the burned areas from the unburned areas, and make it difficult to detect the burned areas when the fire front is expanding. In some cases, such as in a concave region, the fire will merge at the end. How to detect and remove the overlap of the fire becomes a difficult and important problem to solve. Although several methods (Finney [9], Knight and Coleman [16], Osher and Sethian [22], Richards [25], Richards and Bryce [26], Wallace [37]) have been proposed to resolve crossovers problems, crossover remains a challenging issue. Finney [9] states: "regardless of the methods chosen, the process of crossover removal is expensive in time and computing power, and is an interesting area for further research and improvement." Different kinds of crossovers are shown in Fig. 2.1. However Fig. 4.9b shows the level set method can offer a satisfactory solution for the challenging crossover issue.
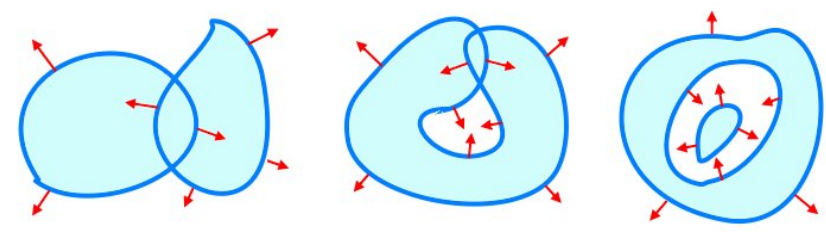

Figure 2.1: Crossover - Different kinds of crossovers.

Another challenge for the vector-based method is the ignition delay time when fire spreads from one type of fuel to a different type of fuel. For example, the heat released 


\section{WILDFIRE MODELS}

from burning grassland may not be intense enough to ignite a woods. On the other hand, fire would spread very quickly from burning woods to grassland. The vector method does not consider the fact that different fuels have different ignition points. Therefore, the fire simulation using the vector method will continue to spread until the fire meets obstacles such as water or rock which has zero ROS.

\subsubsection{Vector-Based Simulation Approach}

For an ideal fire simulation on homogeneous fuel on level terrain without wind, we expect the fire to appear as a circular front, growing in time. Anderson et al. [1] describe this mathematically using the parametric equations

$$
\begin{aligned}
& x=a \cdot t \cdot \cos \vartheta \\
& y=a \cdot t \cdot \sin \vartheta
\end{aligned}
$$

where $x$ and $y$ are the coordinates in the plane of a point on the fire front, $a$ is the rate of spread (uniform across the whole front in this idealized, isotropic situation), $t$ is the time elapsed since ignition, and $\vartheta$ is a parameter which can be interpreted as an angular coordinate determining the location of the front at angles between $0^{\circ}$ and $360^{\circ}$ from the x-axis. With this model, the radius of the circle increases linearly with time at a rate determined by $a$ which is determined by fuel type, temperature, and moisture content, etc. Anderson et al. [1] suggest that it is reasonable to include the effect of a constant wind to further yield an elliptical fire front so that

$$
\begin{aligned}
& x=a \cdot t \cdot(f(U) \cdot \cos \vartheta+g(U)) \\
& y=a \cdot t \cdot(h(U) \cdot \sin \vartheta)
\end{aligned}
$$

where $f, g$, and $h$ are functions depending upon wind speed $U$.

This model fits in with the generally accepted concept that two-dimensional fire shapes are assumed to be generally elliptical under the conditions when factors such as fuel, weather, topography affecting fire behavior are spatially and temporally constant.

Based on this model F.E.Fendell and Wolff [8] focused on the fire front velocities at the head, back, and flanks where propagation is with, against, and across the wind (Fig. 2.2). F.E.Fendell and Wolff [8] proposed the spread speed as 
- Head: $h(U)=\varepsilon_{0}+a \sqrt{U}$

- Flank: $f(U)=\varepsilon_{0}+c_{1} U \exp \left(-c_{2} U\right)$

- Rear: $\varepsilon(U)=\varepsilon_{0} \exp \left(-\varepsilon_{1} U\right)$

where $\varepsilon_{0}, \varepsilon_{1}, c_{1}, c_{2}$ and $a$ are parameters depending on fuel bed and wind velocity $U$.

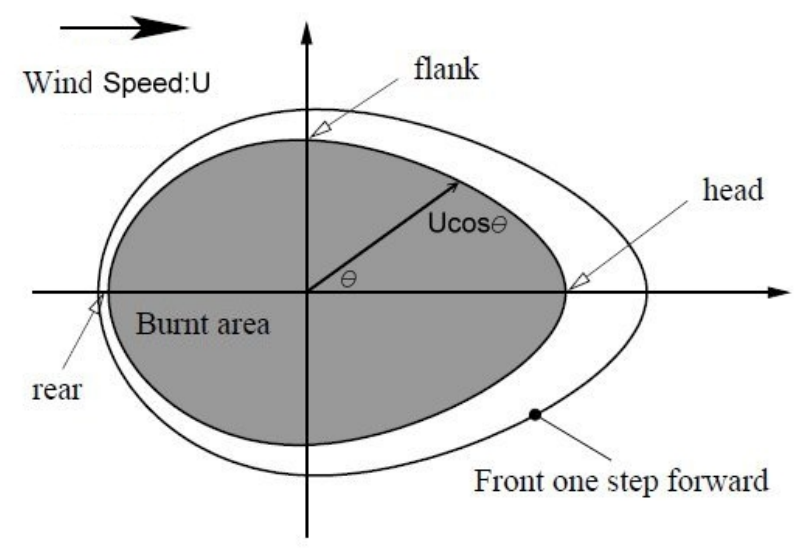

Figure 2.2: An Elliptical Shape Fire-spread Assumption - A one step forward elliptical fire shape simulated under west wind assuming homogeneous fuel and without terrain. [19]

Applying the velocity by trigonometric interpolation for any points, we get spread speed $F$ as

$$
\begin{array}{ll}
F(U, \vartheta)=f\left(U \sin ^{m} \vartheta\right)+h\left(U \cos ^{n} \vartheta\right), & \text { if }|\vartheta| \leq \frac{\pi}{2} \\
F(U, \vartheta)=f\left(U \sin ^{m} \vartheta\right)+\varepsilon\left(U \cos ^{2} \vartheta\right), & \text { if }|\vartheta|>\frac{\pi}{2}
\end{array}
$$

where $\vartheta$ is the angle between the wind direction and the normal to the front. Further, Mallet et al. [19] simplified Eq. 2.9) without losing its main features to

$$
\begin{array}{ll}
F(U, \vartheta)=\varepsilon_{0}+a \sqrt{U \cos ^{n} \vartheta}, & \text { if }|\vartheta| \leq \frac{\pi}{2} \\
F(U, \vartheta)=\varepsilon_{0}(\alpha+(1-\alpha)|\sin \vartheta|), & \text { if }|\vartheta|>\frac{\pi}{2}
\end{array}
$$

where $\varepsilon_{0}, a$ are parameters depending on fuel and $\alpha \in[0,1]$ is the ratio between the velocity at the rear and flanks. Based on empirical field tests and plausibility, Mallet et al. [19] suggested setting $n=3$ to produce a significant, elliptical fire shape from the flanks to the head. Simulation run from our modified Rothermel's model (Eq. (3.7) using level set method produced an ideal fire propagation shape as shown in Fig. 4.2a by applying the same parameters as in Mallet et al. [19]. Our simulation result from 


\section{WILDFIRE MODELS}

level set method matches Mallet et al. [19]'s result.

The simulation proceeds at the following steps at each time interval: The expansion of the fire polygon is computed from its vertices by applying a fire model such as Eq. (2.10) to calculate the direction and distance traveled by the fire at each vertex. After completing the calculation for the fire expansion, the new vertex is inserted into the fire front if the resolution of the perimeter is lower.

For example, FARSITE[9], a widely used fire simulation model by U.S. federal and state land management agencies for predicting fire spread across the landscape, uses two simulation parameters to control the spatial calculations, (1) the distance resolution and (2) the perimeter resolution. The distance resolution is the resolution in the fire spread direction. When the fire perimeter expands and the vertices are separated over time, a new vertex is inserted at the mid-span of a perimeter segment if the distance between the vertices is greater than the resolution of the perimeter. The perimeter resolution is the maximum distance allowed between vertices of the fire polygon. 


\section{3}

\section{Methodologies}

We store fire data at each cell in a lattice, and we apply the Rothermel's fire model to calculate the travel distance of fire based on the local data from the burning cell to the unburned neighboring cells. In order to calculate the next expanding direction and distance from the fire front, we apply Huygens' principle and elliptical spread assumption. Further we apply the level set method which adds one more dimension to solve the original problem. By applying the level set method, we need to focus only on the fire expansion on the norm of the fire front and keep the ellipse shape of fire. We then build a fire simulation model applying distance function to determine the state of cells to simulate the fire spread. We also discuss fast marching method and its implementation. Fast marching method uses the same idea as level set method to add time dimension to the original problem. Instead of calculating the travel distance, fast marching calculates the travel time of the fire surface to the next place.

\subsection{Strategy and Solution}

Pre-existing fire data store at cells provide the advantage to simulate the fire spread especially in a complex environment under the conditions of heterogeneous fuel and weather conditions [33]. However, Ian [15] found that vector-based simulations produce a much more realistic fire perimeter. Sullivan [33] pointed out that the vector-based models are preferred to produce meaningful simulations. Our research is intended to find a method which can simulate fire spread under heterogeneous fuel and produce a 


\section{METHODOLOGIES}

realistic fire perimeter at the same time.

\subsubsection{Strategy}

The fire shape can be replicated exactly by a distance function which at a given point returns the distance to the fire front. We store the distances information at cells and define the distance to be positive for unburned cells and negative for burning and burned cells. When the sign of the distance changes, the state of cells will be updated. We can leverage the level set method and Rothermel's fire model to calculate the fire spread distance and direction, depending on the elliptical spread assumption at sequential time intervals. The advantage of this method is relying only on the fire spread direction by using the elliptical spread assumption and rather than by considering the intervening adjacent cells.

We defined a signed distance function which returns the distance from a given point in space to the surface which is to separate the inside and the outside of some object at time $t$. Let the surface be the burning cell, we have the signed distance function as follows:

$$
\phi((i, j), t=0)= \pm d
$$

where $d$ is the distance from the cell $(i, j)$ to the fire front at the time $t=0$. The positive sign is used if the cell $(i, j)$ is outside the closed fire front which is an unburned cell; the negative sign is used if the cell $(i, j)$ is inside the closed fire front which is a burning cell. When the fire front move forward through time, cell(i,j) eventually becomes negative and its state is updated to a burning cell. To calculate the speed of fire spread, we may use Eq. (3.7) as the rate of fire spread to move forward fire front and we leverage the level set equation Eq. (3.13) to get the distance of the fire moving forward at $\Delta t$. The distances of the cells become negative and their states are updated to burning after a certain time.

The steps to simulate fire spread are first to save local data at each cell; second to add a time axis perpendicular to the $x y$-plane, and third to apply a distance function of the level set method to create a $3 \mathrm{D}$ object. The fire spread can be viewed from a 
3D perspective. The fire front becomes the expanding contour of the $3 \mathrm{D}$ object. We only need to apply some numerical methods which have been introduced by Osher and Sethian [22] to calculate the 3D object's moving distance. The projected contour on the $x y$-plane becomes the fire spread. The $3 \mathrm{D}$ object is generated from the distance between burning cells to unburned cells. Another method in the implementation is the fast marching method using the travel time of fire spread to each unburned cell from the nearest burning cell instead of travel distance. In this study, we will be using modified Rothermel's fire spread rate in the level set methods (the narrow band level set method and the fast marching method) and study the comparison.

\subsubsection{Modification of Rothermel's Model}

Mallet et al. [19] applied the level set method to a simplified fire model Eq. (2.10), and obtained an elliptical fire shape similar to the fire at Fig. 4.2a, Nevertheless, this model can be further studied and modified to fit a more complex environment such as under different kinds of weather, terrain, fuel bed, etc. The model Mallet et al. [19] used has only two conditions including fuel and wind factors. They are part of the same series of factors of Rothermel's model. We kept all the factors of the Rothermel's model except replacing the wind factor with Mallet's model to produce two dimensional simulation under the flank and back wind direction.

We briefly revisited Rothermel's Model Eq. 2.5 on page 11. This model has two characteristics. First, the fire spread rate focuses on the fire heading direction only. Second, this model can be decomposed into three independent parts. For the 'head fire direction only' characteristic, it is necessary to assume that the fire spread shape is elliptical. Otherwise we cannot estimate the fire spread direction except for the head fire. Under this assumption, Mallet's parameters provide a good solution if we consider the wind factor only. We only need the fire speed for head wind and use Mallet's parameters to calculate and predict the speed of the expanding fire in the other directions.

From Rothermel's model Eq. 2.5), if we write the fuel factor $\frac{I_{R} \xi}{\rho_{b} \varepsilon Q_{i g}}$ to a function $f$ and denote $E_{0}=f\left(I_{R}, \xi, \rho_{b}, \varepsilon, Q_{i g}\right)$, then Rothermel's fire model can be simplified to 


\section{METHODOLOGIES}

$$
R=E_{0}\left(1+\Phi_{w}+\Phi_{s}\right)
$$

where $\Phi_{w}$ is factor of wind in Eq. (2.6) and $\Phi_{s}$ is factor of terrain in Eq. (2.7) on page 12 Similarly, we simplify the wind factors $\Phi_{w}$, and write $A=g\left(C, \beta, \beta_{o p}, E\right)$ which $g=C\left(\frac{\beta}{\beta_{o p}}\right)^{-E}$ and let $U^{\prime}=3.28 U$. Then, Eq. (3.1) can be rewritten to

$$
R=E_{0}\left(1+A \cdot U^{B}+\Phi_{s}\right)
$$

If a fire is on the zero elevation with $\vartheta=0$, then $\Phi_{s}=5.275 \beta^{-0.3}(\tan \vartheta)^{2}=0$. Eq. 3.2 becomes

$$
R=E_{0}\left(1+A \cdot U^{\prime B}\right) .
$$

This fire spread depends on fuel $E_{0}$ and wind $A U^{\prime B}$ only.

We next transform Mallet's model[19] Eq. 2.10) on page 15 to a new form

$$
\begin{array}{ll}
F(U, \vartheta)=\varepsilon_{0}+a U^{1 / 2}(\cos \vartheta)^{3 / 2}, & \text { if }|\vartheta| \leq \frac{\pi}{2} \\
F(U, \vartheta)=\varepsilon_{0}(\alpha+(1-\alpha)|\sin \vartheta|), & \text { if }|\vartheta|>\frac{\pi}{2}
\end{array}
$$

Although it has no direct impact, following Mallet's suggestion, we replaced $n$ with 3 here and focused on the headwind $(\vartheta=0)$ only. Mallet's model Eq. (3.4) can be rewritten as

$$
\begin{array}{ll}
F_{H}(U, \vartheta)=\varepsilon_{0}+a U^{1 / 2}, & \text { if }|\vartheta| \leq \frac{\pi}{2} \\
F(U, \vartheta)=\varepsilon_{0}, & \text { if }|\vartheta|>\frac{\pi}{2}
\end{array}
$$

$F_{H}$ is the head wind direction. For any case, Eq. (3.3) and Eq. (3.5) must generate the same results because the equations consist of the same element of fuel and wind to calculate the fire spread. Furthermore, if we consider fuel factor only for the fire-spread simulation, we can assume $E_{0}=\varepsilon_{0}$ to generate the same results. The reason to have the same results from the above two equations is that the equations consist of the same element of fuel. Therefore, we can define a new model as

$$
\begin{cases}R=\varepsilon_{0}+a \sqrt{U \cos ^{3} \vartheta}+\varepsilon_{0} \Phi_{s}, & \text { if }|\vartheta| \leq \frac{\pi}{2} \\ R=\varepsilon_{0}(\alpha+(1-\alpha)|\sin \vartheta|)+\varepsilon_{0} \Phi_{s}, & \text { if }|\vartheta|>\frac{\pi}{2}\end{cases}
$$

and

$$
\Phi_{s}=5.275 \beta^{-0.3}\left(\tan \vartheta_{t}\right)^{2}
$$

An illustration of $R$ vs. $\vartheta$ on the head fire part of this new model is shown at Fig. 3.1 . We set $a=1$ and $U=1$ in this illustration. Head fire part is $-\pi / 2 \leq \vartheta \leq \pi / 2$. 
Traditionally, people use the letter $F$ to represent the velocity vector on the norm direction in the level set method and use the letter $W$ to represent the wind factor in the fire model. We will follow this tradition to use the letter $F$ replacing the letter $R$; use $W(U, \vartheta)$ replacing the wind factor $F(U, \vartheta)$ in the previous equation. In the rest chapters, the fire spread formula in our simulation model will be

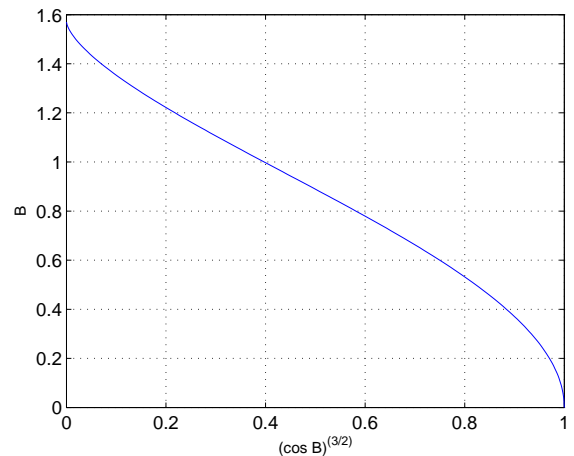

Figure 3.1: Wind coefficient for ROS (dimensionless) - This is an illustration of $R$ vs. $\vartheta$ on the head fire $\left(|\vartheta| \leq \frac{\pi}{2}\right)$ part. $\vartheta$ is the angle between norm of fire front and wind direction in Eq. (3.6).

$$
\begin{cases}F=\varepsilon_{0}+W(U, \vartheta)+\varepsilon_{0} \Phi_{s}, & \text { if }|\vartheta| \leq \frac{\pi}{2} \\ F=\varepsilon_{0}(\alpha+(1-\alpha)|\sin \vartheta|)+\varepsilon_{0} \Phi_{s}, & \text { if }|\vartheta|>\frac{\pi}{2}\end{cases}
$$

where

$$
W(U, \vartheta)=a \sqrt{U \cos ^{3} \vartheta}
$$

and

$$
\Phi_{s}=5.275 \beta^{-0.3}\left(\tan \vartheta_{t}\right)^{2} .
$$

$\vartheta$ is the angle betweeen fire head and wind direction, and $\tan \vartheta_{t}$ is the slope. $\alpha \in[0,1]$ is the ratio between the velocity at the $\operatorname{rear}\left(\alpha \varepsilon_{0}\right)$ and the velocity at the flanks $\left(\varepsilon_{0}\right)$. velocities at the rear and the flanks no longer depend on the wind since their dependence on the wind speed is hard to model accurately and has little impact on the overall front location [19.

Since all fire spread models are developed under the same fuel condition and are not addressed for the fire spread between different fuels, Berjak and Hearne [5] added a combustibility index $C I$ in his raster-based simulation model for heterogeneous environment which is defined as

$$
C I=\frac{H_{\alpha}^{t}}{H_{\beta}}
$$

where

- $H_{\alpha}^{t}$ : the amount of heat produced from $\alpha$ at time $t$ 


\section{METHODOLOGIES}

- $H_{\beta}$ : the total heat required to ignite fuel $\beta$

Because the vector-based simulation models do not consider fire spreading through different types of fuel, we may apply $C I$ to our simulation model for heterogeneous environment and extend the Eq. (3.7) to

$$
\begin{cases}F=\left(\varepsilon_{0}+W(U, \vartheta)+\varepsilon_{0} \Phi_{s}\right) \xi, & \text { if }|\vartheta| \leq \frac{\pi}{2} \\ F=\left(\varepsilon_{0}(\alpha+(1-\alpha)|\sin \vartheta|)\right) \xi, & \text { if }|\vartheta|>\frac{\pi}{2}\end{cases}
$$

where

$$
\begin{gathered}
W(U, \vartheta)=a \sqrt{U \cos ^{3} \vartheta}, \\
\Phi_{s}=5.275 \beta^{-0.3}\left(\tan \vartheta_{t}\right)^{2},
\end{gathered}
$$

and

$$
\xi= \begin{cases}1, & \text { if } C I \geq 1 \\ 0, & \text { if } C I<1\end{cases}
$$

where $C I=\frac{H_{\alpha}}{H_{\beta}}, H_{\alpha}$ is the amount of heat from $\alpha$ and $H_{\beta}$ is the total heat required to ignite $\beta$.

We extend Eq. (3.7) to Eq. (3.9) provided further information on the combustibility of the fire. The incorporation of the combustibility index is motivated by the raster concept. However due to the practicality, we do not use the combustibility index in this thesis. Instead we focus only on Eq. (3.7) in our simulations. However, we provide an artificial example in Sec. 4.3.1 using the combustibility index. The artificial example simulated the fire spread from grasslands to woods. The fire spread slows down from grasslands to woods or in some cases it stops because the ignition of the woods requires more heat than the heat generated from the grasslands.

\subsection{The Level Set Method}

The level set method is introduced by Osher and Sethian [22] to resolve issues from more than one dimensional view. It has become popular in recent years for tracking and simulating the dynamics of a moving surface in 3D or interface in $2 \mathrm{D}$ and it is well known for its ability to easily handle topological changes such as merging or breaking surface or interfaces. The physical evolution is modeled using partial differential 
equations(PDE). The PDE methods for level set evolution are built on the theory of Hamilton-Jacobi equations. To the best of our knowledge, Mallet et al. [19] are the first researchers using the level set method to simulate wildfire spread under wind conditions.

In the level set method, a moving surface can be described by a signed distance function which returns the distance from a given point in space to the surface. Points inside the surface have a negative distance, and those outside have a positive (Fig. 3.2a, Fig. $3.2 \mathrm{~b}$.) distance from the surface. So, the set of points with distance zero, the zero level set, represents the moving surface. That is, a surface is the set $S$ where

$$
S=\left\{x \in R^{n} \mid \phi(x)=0\right\} .
$$

If we add one dimension to represent time and let the values of the distance function depend on time, then $\phi(x, t)$ denotes the distance from the surface at the point $x \in R^{n}$ at time $t \in R^{+}$. We call $\phi(x, t)$ a level set function.

Now we define the level set function of the surface as

$$
S(t)=\left\{x \in R^{n} \mid \phi(x, t)=0\right\}, t \in R^{+}
$$

$\phi(x, t)=0$ is called the zero level set. The zero level set is the contour of the level set function on the $x y$-plane. When the level set function moves over time, the new zero level set forms a new contour. If we constrain the level set value on the zero level set which is the contour with motion $x(t)$, we can obtain the surface change. We want to know the new values for each of the points on the surface as time moves forward. That is, what $\phi_{\text {new }}(x)$ is after $\Delta t$, if we have $\phi_{\text {old }}(x)$.

Using the Euler method, a first-order numerical scheme for solving ordinary differential equations, if we have a differential equation of the form

$$
\begin{aligned}
& y^{\prime}(t)=f(t, y(t)) \\
& \text { at } \quad y\left(t_{0}\right)=y_{0},
\end{aligned}
$$

its one step approximate solution from $t_{n}$ to $t_{n+1}=t_{n}+h$ is

$$
y_{n+1}=y_{n}+h f\left(t_{n}, y_{n}\right) .
$$




\section{METHODOLOGIES}

$5.65695 .0000 \quad 4.47214 .12314 .0000 \quad 4.0000 \quad 4.00004 .12314 .47215 .0000 \quad 5.6569$

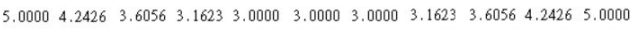
$\begin{array}{lllllllllll}4.4721 & 3.6056 & 2.8284 & 2.2361 & 2.0000 & 2.0000 & 2.0000 & 2.2361 & 2.8284 & 3.6056 & 4.4721\end{array}$ $\begin{array}{lllllllllll}1231 & 3.1623 & 2.2361 & 1.4142 & 1.0000 & 1.0000 & 1.0000 & 1.4142 & 2.2361 & 3.1623 & 4.1231\end{array}$ $\begin{array}{llllllllll}4.0000 & 3.0000 & 2.0000 & 1.0000 & 0 & 0 & 1.0000 & 2.0000 & 3.0000 & 4.0000\end{array}$

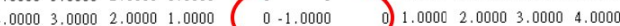

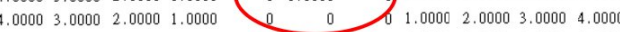
$\begin{array}{lllllllllll}4.1231 & 3.1623 & 2.2361 & 1.4142 & 1.0000 & 1.0000 & 1.0000 & 1.4142 & 2.2361 & 3.1623 & 4.1231\end{array}$ $\begin{array}{llllllllllllll}4.4721 & 3.6056 & 2.8284 & 2.2361 & 2.0000 & 2.0000 & 2.0000 & 2.2361 & 2.8284 & 3.6056 & 4.4721\end{array}$ $\begin{array}{lllllllllllll}5.0000 & 4.2426 & 3.6056 & 3.1623 & 3.0000 & 3.0000 & 3.0000 & 3.1623 & 3.6056 & 4.2426 & 5.0000\end{array}$ $\begin{array}{lllllllllll}5.6569 & 5.20000 & 4.4721 & 4.1231 & 4.0000 & 4.0000 & 4.0000 & 4.1231 & 4.4721 & 5.0000 & 5.6569\end{array}$

(a) A distance function

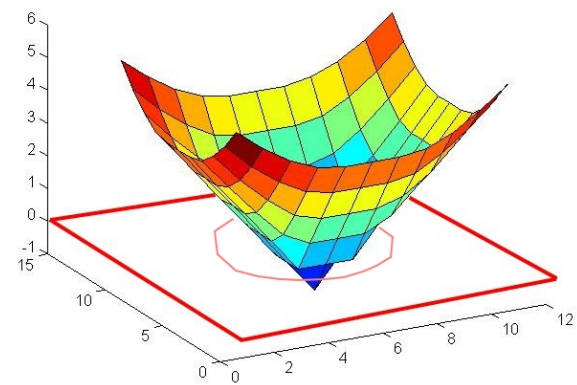

(c) A $3 \mathrm{D}$ view from Fig. 3.2a and its contour on zero level plane

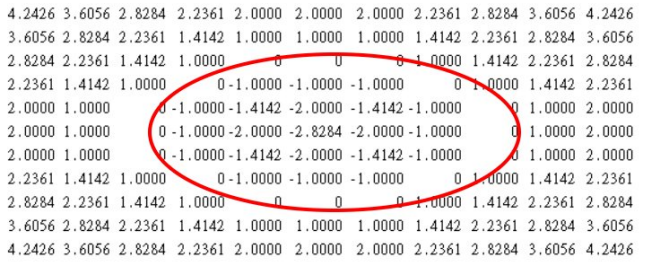

(b) Interface expanding

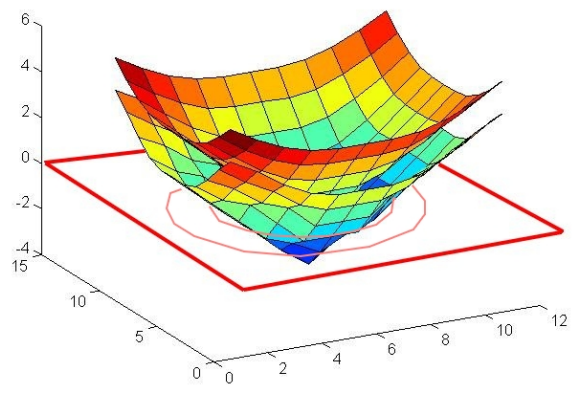

(d) The 3D object sinks down to expand the contour

Figure 3.2: Evolution of a distance function - The 11 x 11 matrix on the xy-plane represents a distance function. The points with value 0 indicate the interface $\phi(x, t)=0$. Inside the interface, the distance from points to interface is negative. Outside is positive. The distance function Fig. $3.2 \mathrm{a}$ expands its surface outwardly to become Fig. 3.2b. The distance number at each point can also be seen as a slope with 1 on the norm to create a $3 \mathrm{D}$ object. Fig. $3.2 \mathrm{c}$ and Fig. 3.2d provide the same distance function of Fig. $3.2 \mathrm{a}$ and Fig. $3.2 \mathrm{~b}$ respectively from the $3 \mathrm{D}$ view. Fig. $3.2 \mathrm{~d}$ shows the contour expanding outside when the $3 \mathrm{D}$ distance function is projected onto the $2 \mathrm{D}$ plane. 
It is

$$
y^{\prime}\left(t_{n}\right)=f\left(t_{n}, y\left(t_{n}\right)\right)=\frac{y\left(t_{n+1}\right)-y\left(t_{n}\right)}{t_{n+1}-t_{n}} .
$$

So following Euler's method, our surface level set function is

$$
\begin{gathered}
\phi^{\prime}(t)=\frac{\partial \phi}{\partial t}=\frac{\phi_{\text {new }}(x)-\phi_{\text {old }}(x)}{\Delta t} . \\
\phi_{\text {new }}(x) \approx \phi_{\text {old }}(x)+\Delta t \cdot \phi^{\prime}(t) .
\end{gathered}
$$

Our goal is to find $\phi^{\prime}(t)$ to get $\phi_{\text {new }}(x)$.

By the chain rule, we apply the ordinary differential equations(ODEs) method to the zero level set Eq. (3.10).

$$
\begin{gathered}
\frac{d}{d t} \phi(x, t)=0 \\
\frac{\partial \phi}{\partial t}+\frac{\partial \phi}{\partial x} \cdot \frac{\partial x}{\partial t}=0 \\
\frac{\partial \phi}{\partial t}+\nabla \phi \cdot \frac{\partial x}{\partial t}=0 \\
\frac{\partial \phi}{\partial t}=-\nabla \phi \cdot \frac{\partial x}{\partial t}
\end{gathered}
$$

Here, $\nabla \phi$ is the gradient of $\phi$ with respect to $x$, and $\frac{\partial x}{\partial t}$ can be seen as the velocity of a particle trajectory $x(t)$ on the surface $\phi(x, t)$. If we let a velocity field $V=x^{\prime}(t)$, then we may rewrite Eq. 3.12 as

$$
\phi^{\prime}(t)=-V \cdot \nabla \phi
$$

We call Eq. (3.13) level set equation. Once we find $\phi^{\prime}(t)$, we may get $\phi_{\text {new }}$ from Eq. (3.11).

For fire front propagation, based on Huygens' principle, the vector field moves forward in the normal direction of the surface. Assuming at a given point $x \in R^{n}$ and the speed $F$ at $x$, the velocity field $V$ used in level set equation Eq. (3.13) will then be defined as

$$
\begin{aligned}
& \qquad F=V \cdot n \\
& \text { where } \quad n=\frac{\nabla \phi}{|\nabla \phi|} .
\end{aligned}
$$

Replace it in Eq. (3.13), and we have 


\section{METHODOLOGIES}

$$
\begin{aligned}
\phi^{\prime}(t) & =-V \cdot \nabla \phi \\
& =-V \cdot \frac{\nabla \phi}{|\nabla \phi|}|\nabla \phi| \\
& =-V \cdot n|\nabla \phi| \\
& =-F|\nabla \phi| .
\end{aligned}
$$

This is the level set equation in Hamilton-Jacobi form. Put Eq. (3.14 back to Eq. (3.11), we get the new zero level set position.

\subsubsection{The Narrow Band Method}

The level set method allows us to trace the moving surface. However, since we are tracing the fire front, the points far way from the zero level set are not in immediate needs, and also the points inside the zero level set are no longer needed. The narrow band method which is described in Peng et al. 24] allows us to only solve the level set equation in a narrow band around the surface. All values outside this band are assumed to have a very large or a very small value, dependent on whether they are outside or inside the surface. Compared to the standard level set method, the only extra task of the narrow band is to keep its collection of points updated along with the movement of the surface. Since the narrow band has information only on the neighboring points of the zero level set, computations that involve neighboring points are sent through that narrow band. The narrow band is represented as a matrix $N B$. The values in the matrix are $-1,0,1$ in which -1 means inside the narrow band, 0 means in the narrow band, and 1 means outside the narrow band. It is used as a pointer to level set function, and the evolution of level set function only happens at the 0 pointers. The narrow band method can reduce the computational complexity of level set evolution from $\bigcirc\left(n^{2}\right)$ to $\bigcirc(n)$ at a time step [24].

\subsubsection{Numerical Solution for Narrow Band Method}

We use the level set method to simulate a two dimensional fire front propagation. So we may consider $\nabla \phi$ at the Hamilton-Jacobi equation Eq. 3.14 as $\nabla \phi=\left(\frac{\partial \phi}{\partial x}, \frac{\partial \phi}{\partial y}\right)$. 
Then we use the upwind scheme to approximate the value at each point of $\phi$. Given a point $(x, y) \in R^{2}$ and a vector $v=\left(v_{x}, v_{y}\right)=V(x, y)$, the upwind scheme dictates that

$$
\frac{\partial \phi}{\partial x} \approx \begin{cases}\phi_{x}^{+}=\phi(x+\triangle x, y)-\phi(x, y), & v_{x}<0 \\ \phi_{x}^{-}=\phi(x, y)-\phi(x-\triangle x, y), & v_{x}>0\end{cases}
$$

and the same for

$$
\frac{\partial \phi}{\partial y} \approx \begin{cases}\phi_{y}^{+}=\phi(x, y+\triangle y)-\phi(x, y), & v_{y}<0 \\ \phi_{y}^{-}=\phi(x, y)-\phi(x, y-\triangle y), & v_{y}>0 .\end{cases}
$$

For Eq. 3.14,

$$
\phi^{\prime}(t)=-F|\nabla \phi|
$$

where

$$
|\nabla \phi|=\sqrt{\left(\frac{\partial \phi}{\partial x}\right)^{2}+\left(\frac{\partial \phi}{\partial y}\right)^{2}} .
$$

Now, we only need to find the value of the gradient. One way of doing this is to use Godunov's method. This method dictates that

$$
\left(\frac{\partial \phi}{\partial x}\right)^{2} \approx \begin{cases}\max \left(\max \left(\phi_{x}^{-}, 0\right)^{2}, \min \left(\phi_{x}^{+}, 0\right)^{2}\right), & v_{x}<0 \\ \max \left(\min \left(\phi_{x}^{-}, 0\right)^{2}, \max \left(\phi_{x}^{+}, 0\right)^{2}\right), & v_{x}>0\end{cases}
$$

where $\phi_{x}^{-}$and $\phi_{x}^{+}$are defined as in Eq. 3.15. The same as

$$
\left(\frac{\partial \phi}{\partial y}\right)^{2} \approx \begin{cases}\max \left(\max \left(\phi_{y}^{-}, 0\right)^{2}, \min \left(\phi_{y}^{+}, 0\right)^{2}\right), & v_{y}<0 \\ \max \left(\min \left(\phi_{y}^{-}, 0\right)^{2}, \max \left(\phi_{y}^{+}, 0\right)^{2}\right), & v_{y}>0 .\end{cases}
$$

$\operatorname{After}\left(\frac{\partial \phi}{\partial x}\right)^{2}$ and $\left(\frac{\partial \phi}{\partial y}\right)^{2}$ are calculated, they can be used to find $|\nabla \phi|$. With this we can get $\phi_{t}^{\prime} \approx-F \cdot|\nabla \phi|$ and then use it to find $\phi_{\text {new }}$, by using the Euler method Eq. 3.11.

Euler's method is a discretized numerical method, and we need to apply the CFL (Courant-Friedrichs-Lewy) condition to keep confirming the stability for our numerical methods. CFL states that, given a space discretization, a time step must be smaller than a computable quantity. If a time step causes the change in distance of a point to be larger than the grid size, it will make the numerical computation unstable. So, the maximum time step $\Delta t$ should be limited for the fastest point in the vector field to travel through the side length of cell in the grid, i.e.

$$
\frac{\triangle t \cdot \max \{|v|\}}{\triangle x} \leq \alpha
$$




\section{METHODOLOGIES}

where $0<\alpha<1$. In the two-dimensional case, the CFL condition becomes

$$
\frac{\triangle t \cdot \max \left\{\left|v_{x}\right|\right\}}{\triangle x}+\frac{\triangle t \cdot \max \left\{\left|v_{y}\right|\right\}}{\triangle y} \leq \alpha,
$$

where $v_{x}, v_{y}$ is the velocity on $x$-axis, $y$-axis respectively. As mentioned, the zero level set has the Hamilton-Jacobi's form $\phi_{t}+H\left(\phi_{x}, \phi_{y}\right)=0$ and Hamilton-Jacobi for CFL constraint is

$$
\triangle t \cdot \max \left\{\frac{\left|H_{x}\right|}{\triangle x}+\frac{\left|H_{y}\right|}{\triangle y}\right\}<\alpha, \quad 0<\alpha<1
$$

where

$$
\begin{aligned}
& H_{x}=F \frac{\phi_{x}}{|\nabla \phi|} \\
& H_{y}=F \frac{\phi_{y}}{|\nabla \phi|}
\end{aligned}
$$

for $H=F \nabla \phi$. We can use this CFL condition to set $\triangle t$ at Eq. 3.11.

\subsubsection{The Algorithm of Narrow Band Method}

The narrow band level set method is the following,

1. Initialize $\phi_{i}=\phi_{\text {old }}$

2. Update narrow band $N B$ and $\phi=\phi_{i}$ at pointer $N B=0$

3. Evaluate derivative $\phi^{\prime}$

(a) Evaluate plus derivatives $\phi^{+}$and minus derivatives $\phi^{-}$from Eq. (3.15)

(b) Select $\phi^{\prime}$

i. Effective $\mathrm{F}$ in its dimension $\left(V \phi^{-}=F * \phi^{-}\right.$and $\left.V \phi^{+}=F * \phi^{+}\right)$.

ii. Determine the upwind direction.

$$
\begin{aligned}
& \text { if } V \phi^{-} \& V \phi^{+} \leq 0 \text { take } \phi^{+} \\
& \text {if } V \phi^{-} \& V \phi^{+} \geq 0 \text { take } \phi^{-} \\
& \text {if } V \phi^{-}>0 \& V \phi^{+}<0 \\
& \quad \text { if } V \phi^{-}>V \phi^{+} \text {take } \phi^{-}, \text {otherwise take } \phi^{+} \\
& \text {if } V \phi^{-}<0 \& V \phi^{+}>0 \text { take } V / F
\end{aligned}
$$

4. Get gradient $|\nabla \phi|$ from Eq. 3.18 
5. Get $H_{x}, H_{y}$ from Eq. 3.20

6. Get $\triangle t=\left(\max \left\{\frac{\left|H_{x}\right|}{\triangle x}+\frac{\left|H_{y}\right|}{\triangle y}\right\}\right)^{-1} * \alpha$ from Eq. (3.19)

7. Get new $\phi_{\text {new }} \approx \phi_{\text {old }}+\Delta t \cdot \phi^{\prime}$ from Euler's forward method

\subsubsection{Strategy for Wildfire Simulation Using the Narrow Band Level Set Method}

Since we only consider two space dimensions for the fire spread model, we apply the level set method on the $x y$-plane. We apply a function $\phi(x, y, t)$ to the space which the interface inhabits, where $(x, y)$ is a point in $x y$-plane, and $t$ is a point in time axis. The function is initialized at $t=0$, and then used to approximate the value of $\phi(x, y, t)$ over time increments. In general, we choose the distance function as our function $\phi$. At any time $t$, the evolving curve, the fire front in our simulation, corresponds to the locus of all points $(x, y)$ such that $\phi(x, y, t)=0$, and that locus is a level curve of the function $\phi$. The locus of all points $(x, y)$ such that $\phi(x, y, t)=d$, is a contour around the original curve at $t=0$, where $d$ is an arbitrary positive or negative constant. Next we need to find the force $F$ on the normal direction to the zero level set. We use $F$ as the rate of fire spread.

To formulate the rate of fire spread $(F)$, we apply the polygon method and make two assumptions. Under the first assumption, we use Huygens' principle that each vertex on the fire front is independent of the others and would expand in the same way as those from the original fire front. Therefore, we can repeatedly use the same fire spread formula to estimate the next ignition point. The second assumption is that the shape of the fire front is elliptical. From this assumption, we may use the fire forward formula to calculate the fire expanding speed for other directions in addition to the head fire by certain geometrical properties. Rothermel's fire model, Eq. (2.5), focuses on the heading portion of a fire from a real observed fire. The velocity from Rothermel's model is based on the head fire on a straight line. We use Rothermel's fire model and replace the wind factor part with the wind part suggested by Mallet et al. [19] for our fire simulation model $F$ for an elliptical expanding fire shape. 


\section{METHODOLOGIES}

\subsubsection{Methodology Steps}

Our approach proceeds in five steps:

In the first step we scale the actual area of simulation into the computational domain and discretize the domain with a uniform mesh grid size. This is the area where we want to simulate the fire propagation.

In the next step, we save the parameters describing the fuel content (for $\varepsilon_{0}$ ) and elevation (for $\Phi_{s}$ ) at that particular location for each cell to form a same size of matrix as of the domain at the last step.

In the third step we initialize the value of a distance function $\phi(x, y, t)$ at the mesh. This is the starting point of the fire. The function $\phi$ is defined as $\phi(x, y, t)= \pm d$ where $d$ is the distance from the point $(x, y)$ to the interface at time $t=0$. The positive sign is used for points $(x, y)$ outside of the closed curve which represent unburned area; the negative sign is used inside of the closed curve representing burned area.

In the fourth step we use Eq. (3.7) as a vector field $F$ for points $(x, y)$ advecting in the direction decided by elliptical spread assumption. Calculate the $F$ from the matrix at step two and wind parameter.

Finally we use the level set method discussed in Section 3.2 .1 .2 to get the new position for $\phi$ at time $t_{\text {new }}$.

The pseudo-code is shown in table 3.1 .

\subsection{Fast Marching Method}

The fast marching method is another method that uses higher dimensions to resolve a problem in lower dimensions. This method is built upon the level set concept initiated by Sethian [31]. The level set method creates a 3D object from the distance to the surface for each point, and the fast marching method creates a 3D object from the travel time at each point. From the level set method, we have the level set function

$$
z=\phi(x, y, t)
$$

contour function at time $t$

$$
0=\phi(x, y, t)
$$

and level set PDE

$$
\phi_{t}=-F|\nabla \phi| .
$$


Table 3.1: Level set pseudo-code - Level set pseudo-code for fire spread simulation.

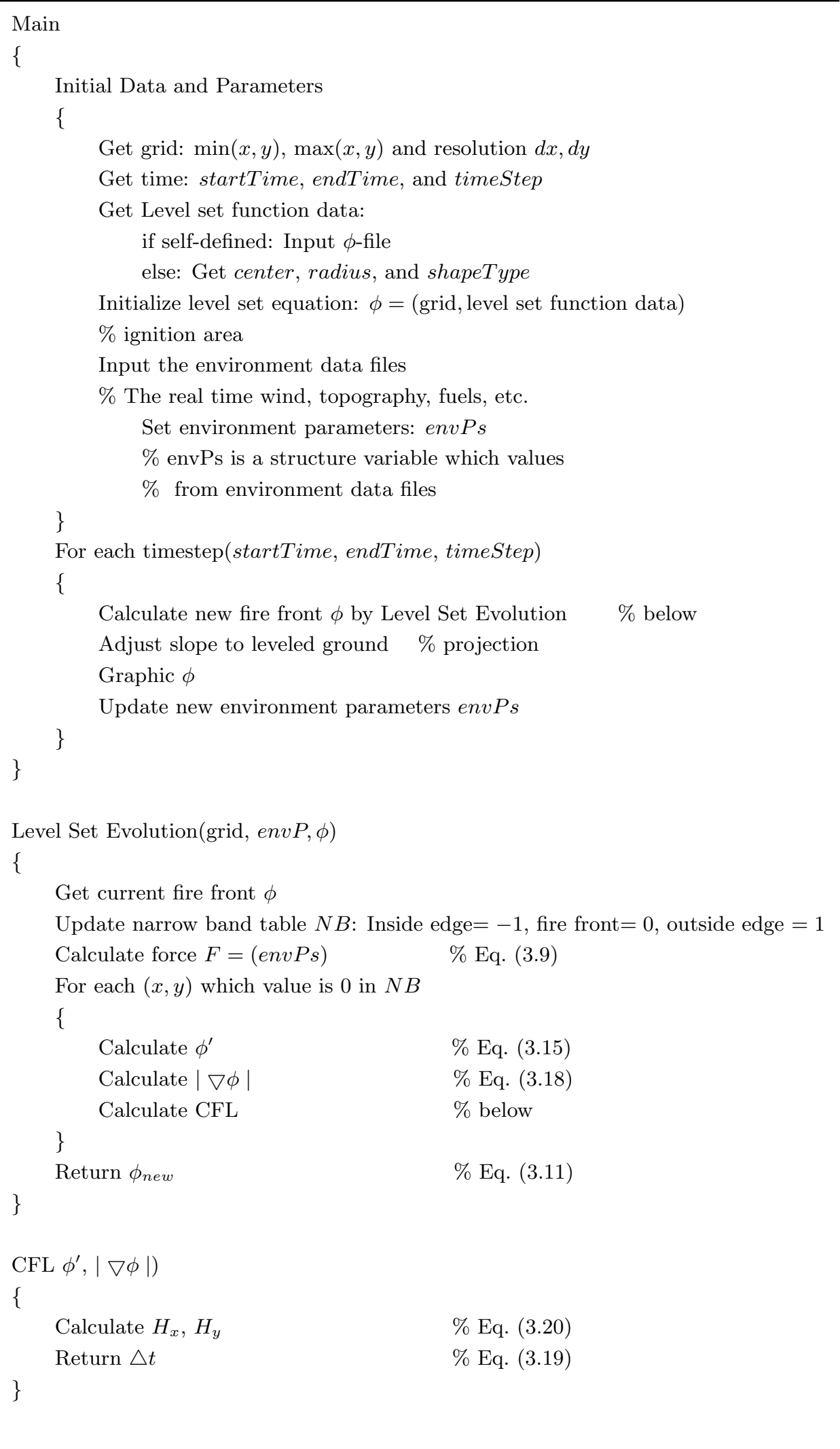




\section{METHODOLOGIES}

We assume the speed $F$ never changes sign, that is, the contour is either always expanding $(F>0)$ or always shrinking $(F<0)$. This monotonicity assumption guarantees the contour crosses each grid point $(x, y)$ only once; thus the level set problem $z=\phi(x, y, t)$ can be converted into a problem to compute $T(x, y)=t$ which $T$ is the set of time which the contour is supposed to cross the grid point $(x, y)$ at time $t$. That is, the contour at time $t$,

$$
t=\{(x, y) \mid T(x, y)=t\}
$$

We compute $T$ using the distance formula Distance $=$ Velocity $*$ Time, which is

$$
\begin{cases}1=F * d T / d x, & \text { for } 1 \mathrm{D} \\ 1=F *|\nabla T|, & \text { for } 2 \mathrm{D}\end{cases}
$$

Let $u_{(x, y)}$ be the travel time of the contour front to point $(x, y)$ on $x y$-plane. Contour $0=\phi(x, y, t)$ becomes single-valued in $t$. This leads to the fast marching equation

$$
\left|\nabla u_{(x, y)}\right| F=1
$$

All $u(x, y)$ constitute the travel time matrix T. Eq. (3.21) may be described by the Eikonal equation,

$$
\left(\frac{\partial u}{\partial x}\right)^{2}+\left(\frac{\partial u}{\partial y}\right)^{2}=1 / F^{2}
$$

This equation combined with some optimal sorting technique results in a very fast solution. Sethian [31] suggested an optimal sorting technique which is modified from Dijkstra's Method [7] to solve Eq. 3.22 by simulating the advancing surface. Every point on the computational grid is classified into three groups: Alive - points inside the surface, whose travel times are known and won't be changed; NarrowBand - points on the surface, whose travel times have been calculated and are waiting to be fixed; and Far - points outside the surface.

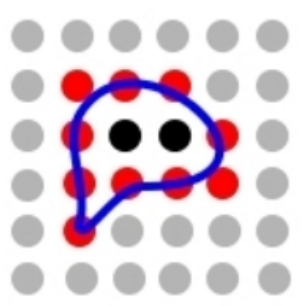

Figure 3.3: Narrow band surface - An advancing surface with the 'narrow band' points (red) on the front and 'alive' points (black) inside; outside are 'far' points (gray).

Fig. 3.3 shows an advancing surface with 'narrow band' points on the front, 'alive' points inside and 'far' points outside of the surface. The algorithm proceeds as following: 


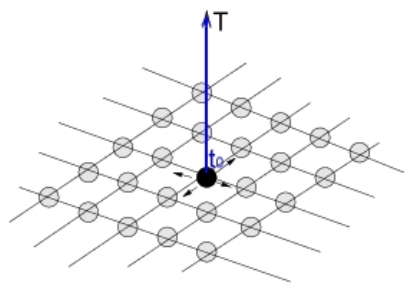

(a) Update unvisited neighboring cells

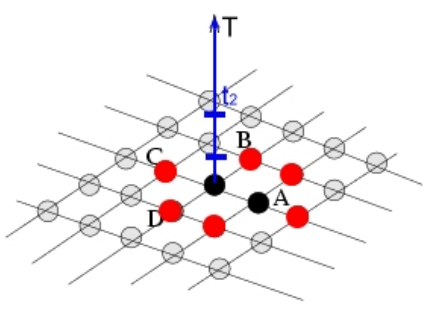

(d) Update neighboring cells

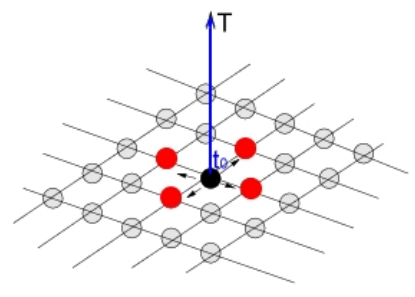

(b) Compute new possible travel time

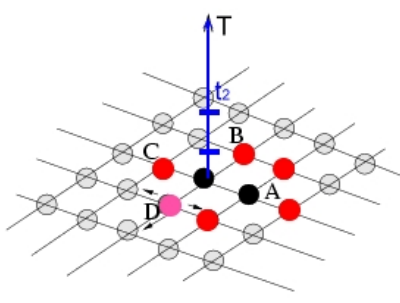

(e) Expand point on the fringe with minimum value

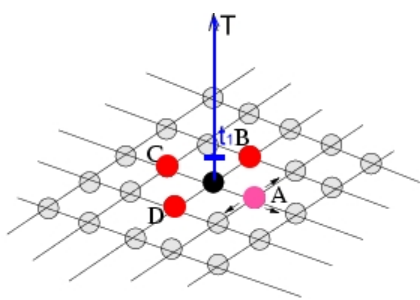

(c) Expand point on the fringe with minimum value

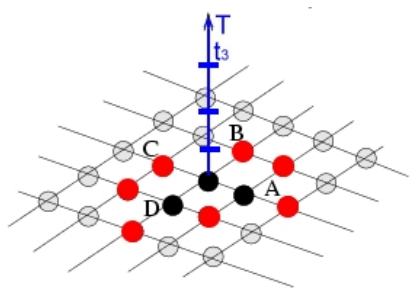

(f) Update neighboring cells

Figure 3.4: Fast Marching Algorithm - A fast marching algorithm to evolute the surface on 2D plane [31]. Black points are 'alive' points, red points are 'narrow band' points, gray points are 'far' points. A pink point in Fig.(c) and Fig.(e) is a narrow band point and is changed to an alive point.

1. Choose the points on the NarrowBand with the smallest travel time.

2. Move those points to the Alive group and advance the surface.

3. Pick points on the fringe of the new Alive to NarrowBand.

4. Update travel times for the new NarrowBand points by solving Eq. (3.22) numerically.

5. Repeat previous steps until all points are in the Alive group.

Fig. 3.4 modified from Sethian [31] shows an example of the fast marching solution procedure. 


\section{METHODOLOGIES}

\subsubsection{Numerical Solution for Fast Marching}

To solve Eq. (3.22) on procedure step 4 in the previous section, Tsitsiklis [36] first described an optimal-control approach, while Sethian [31] developed these techniques based on upwind numerical schemes. The schemes to get the viscosity solution of Eq. (3.22) is obtained by

$$
\begin{aligned}
& \max \left(\max \left(u_{i, j}-u_{i-1, j}, 0\right),-\min \left(u_{i+1, j}-u_{i, j}, 0\right)\right)^{2} \\
+ & \max \left(\max \left(u_{i, j}-u_{i, j-1}, 0\right),-\min \left(u_{i, j+1}-u_{i, j}, 0\right)\right)^{2}=1 / F^{2}
\end{aligned}
$$

That is,

$$
\max \left(u_{i}^{-}, 0\right)^{2}+\min \left(u_{i}^{+}, 0\right)^{2}+\max \left(u_{j}^{-}, 0\right)^{2}+\min \left(u_{j}^{+}, 0\right)^{2}=1 / F^{2}
$$

where $u_{i}^{-}$is a backward $x$ difference for time $t$ at grid point $(i, j), u_{i}^{+}$is a forward $x$ difference, and $u_{j}$ are defined similarly. The roots of the quadratic equation, $a t^{2}+b t+$ $c=0$ can be calculated as

$$
t=\frac{-b \pm \sqrt{b^{2}-4 a c}}{2 a}
$$

Solving Eq. (3.23) amounts to accumulating coefficients $a, b$ and $c$ from its non-zero terms, and evaluating $t$ with Eq. (3.24). For example, if we choose the first term of Eq. (3.23) to calculate.

$$
\begin{gathered}
u_{i}^{-}=\frac{t_{i, j}-t_{i-1, j}}{\triangle i} \\
\text { then }\left(u_{i}^{-}\right)^{2}=\frac{t_{i, j}^{2}-2 t_{i-1, j} t_{i, j}+t_{i-1, j}^{2}}{\triangle i^{2}} \\
\left(u_{i}^{-}\right)^{2}=\alpha t_{i, j}^{2}-\beta t_{i, j}+\gamma
\end{gathered}
$$

where $\alpha=\frac{1}{\triangle i^{2}}, \beta=-2 t_{i-1, j} \alpha$, and $\gamma=t_{i-1, j}^{2} \alpha$. Coefficients $a, b$, and $c$ of Eq. 3.24 can now be accumulated from

$$
\begin{aligned}
& a=\sum_{k} \alpha_{k}, \\
& b=\sum_{k} \beta_{k}, \\
& c=\sum_{k} \gamma_{k} .
\end{aligned}
$$

where index $k$ refers to each term in Eq. (3.23) and subject to the various min / $\max$ conditions.

Because $u_{i}^{ \pm}$is a one step forward/backward, $x$ derivative for time $t$ at grid point $(i, j)$ by the first order upwind, this two point formula is only first-order accurate. If 
we choose three points or more, we may expect the method to have second-order or higher accuracy depending on the algorithm from Rickett and Fomel [27].

\subsubsection{Fast Marching Methodology Steps}

Algorithm of the fast marching method is as follows:

1. Initialize the travel time discretized matrix $T$

- points on the surface are 0 ;

- points in the surface are -1 ;

- points out of the surface are $\infty$.

2. Initialize the narrow band matrix $N B$

- $N B(x, y)=-1$ if $T(x, y)<0$;

- $N B(x, y)=0$ if $T(x, y)=0$;

- $N B(x, y)=1$ if $T(x, y)>0$.

3. Initialize forward speed matrix $F$ which is Eq. 3.9

4. Update $T(x, y)=d x / F(x, y)$ at $N B(x, y)=0$ where $d x$ is resolution of landscape

5. Update $N B$ at $N B(x, y)=0$

- $N B(x, y)=-1$ at $\min T(x, y)$;

- $N B(x \pm 1, y)=0$ if $N B(x \pm 1, y)=1$;

- $N B(x, y \pm 1)=0$ if $N B(x, y \pm 1)=1$.

6. Update $F$ at $N B(x, y)>0$

7. Update $T$ at $N B(x, y)=0$

8. Solve Eq. (3.22) by Eq. (3.23) from Eq. (3.24), Eq. (3.25) and Eq. (3.26)

9. Update $T$ at $N B(x, y)=0$ from the solution of Eq. 3.22

10. Recompute $T$ and $N B$ until $F$ goes to 0

The pseudo-code for this procedure is shown in table 3.2 . 


\section{METHODOLOGIES}

Table 3.2: Fast marching pseudo-code - Fast marching pseudo-code for fire spread simulation.

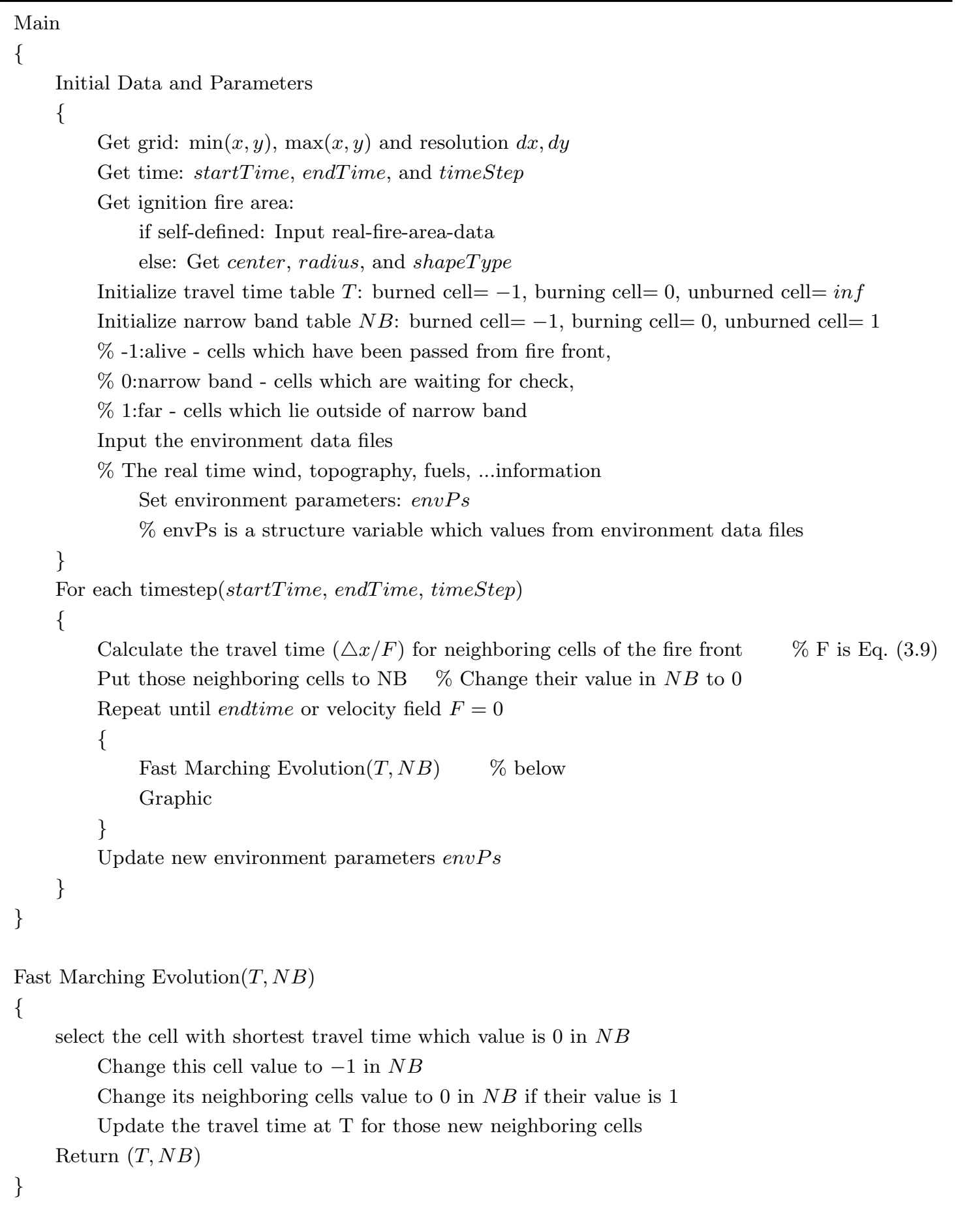




\subsection{Simulation Model}

Our fire simulation is created using MATLAB. Information and newest version about MATLAB are available at the Mathworks[20] website. Our model is designed from an object-oriented framework, in which our model has been split into a number of objects. Objects and their functions are defined as following:

- Grid : an orthogonal square mesh used as the fire field to save the local information, grid size decided by maximum $x$ value, minimum $x$ value, and mesh size $d x$

- Initial level set function : self-defined fire shape function or selected from system defaults;

- Evolution speed and type : defines the fire model;

- Numerical scheme : level set or fast marching;

- Output management: screen display or print out;

- GUI input management : manage the data or parameters such as time period, accuracy,etc.;

- System setting.

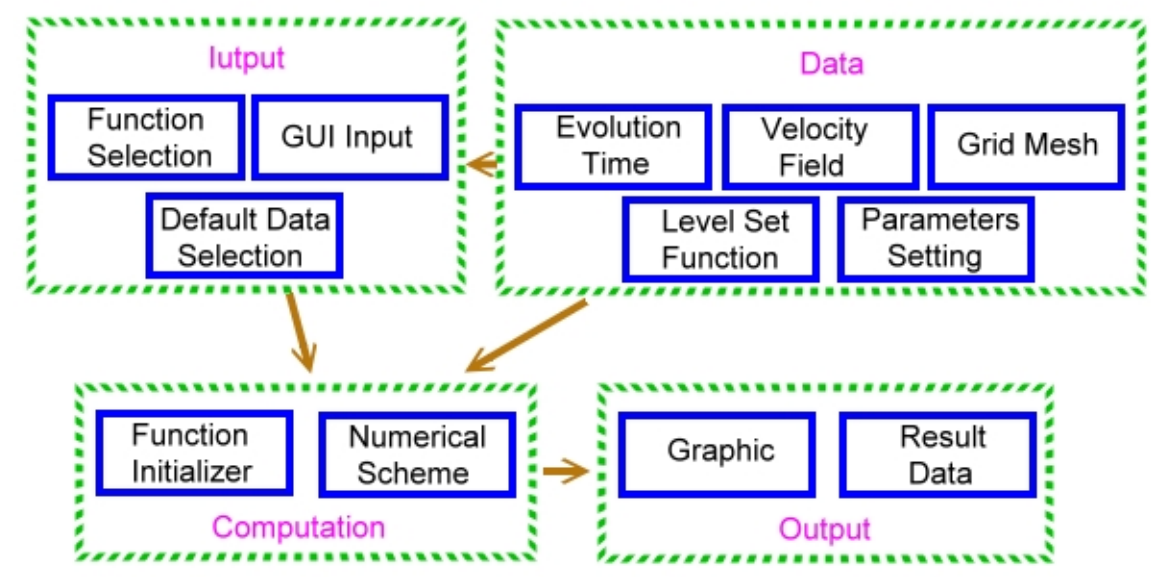

Figure 3.5: Model structure - The simulation model structure. 


\section{METHODOLOGIES}

The structure of the simulation model is shown Fig. 3.5. All adequate fire simulations require data that includes fuel, weather, and topography. In this model, weather and winds are input as data matrices, whereas fuel and topography are provided as GIS raster themes such as in Fig. 5.4 on page 67 for the Troy fire simulation and Fig. 5.13 on page 74 for the Colina fire simulation. Self-defined vector data could also be used but requires access to the local information for each cell. Raster resolutions of 25 to $50 \mathrm{~m}$ are most commonly used for landscapes. Wind direction is represented in a vector such as [-1 0 [ for east wind, [ [1 1 ] for north east wind, etc. Fig. 3.6 shows the level set model and the fast marching model running with the same parameters.

All model verifications and fire simulations in this study are run on a personal computer with an AMD Athlon $64 \times 2$ dual-core processor with $3072 \mathrm{MB}$ system memory. 


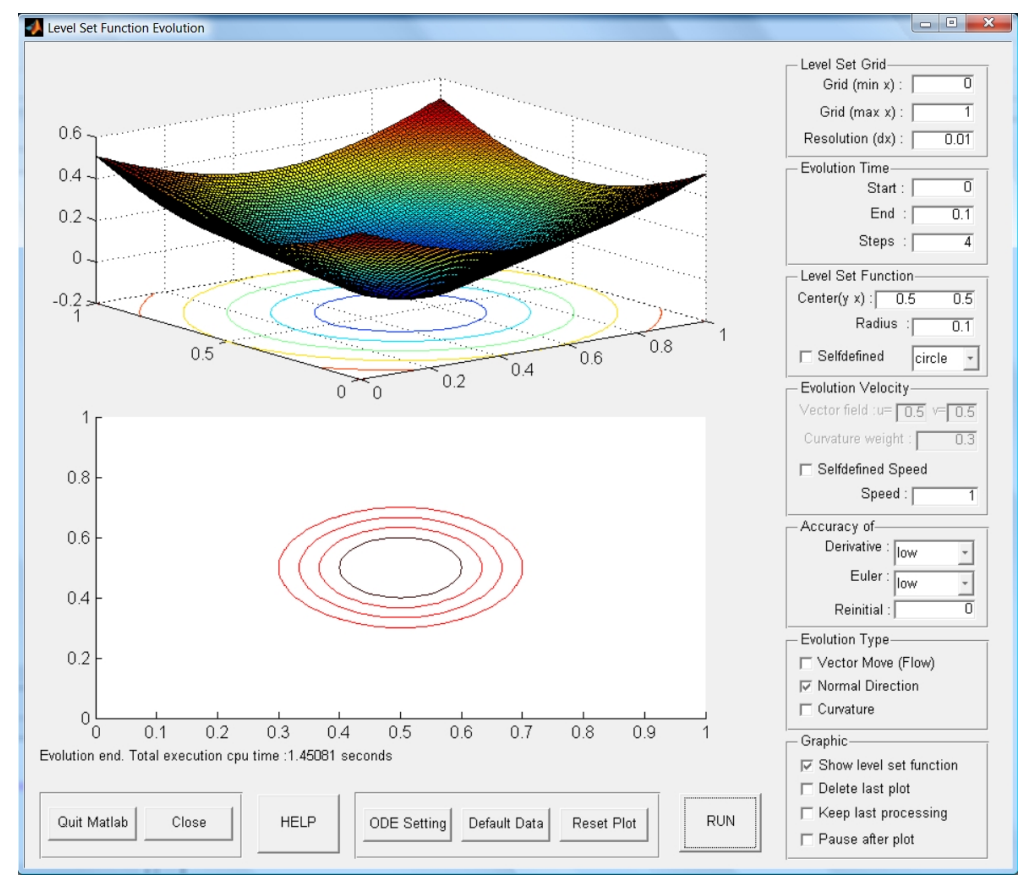

(a) Example for level set method

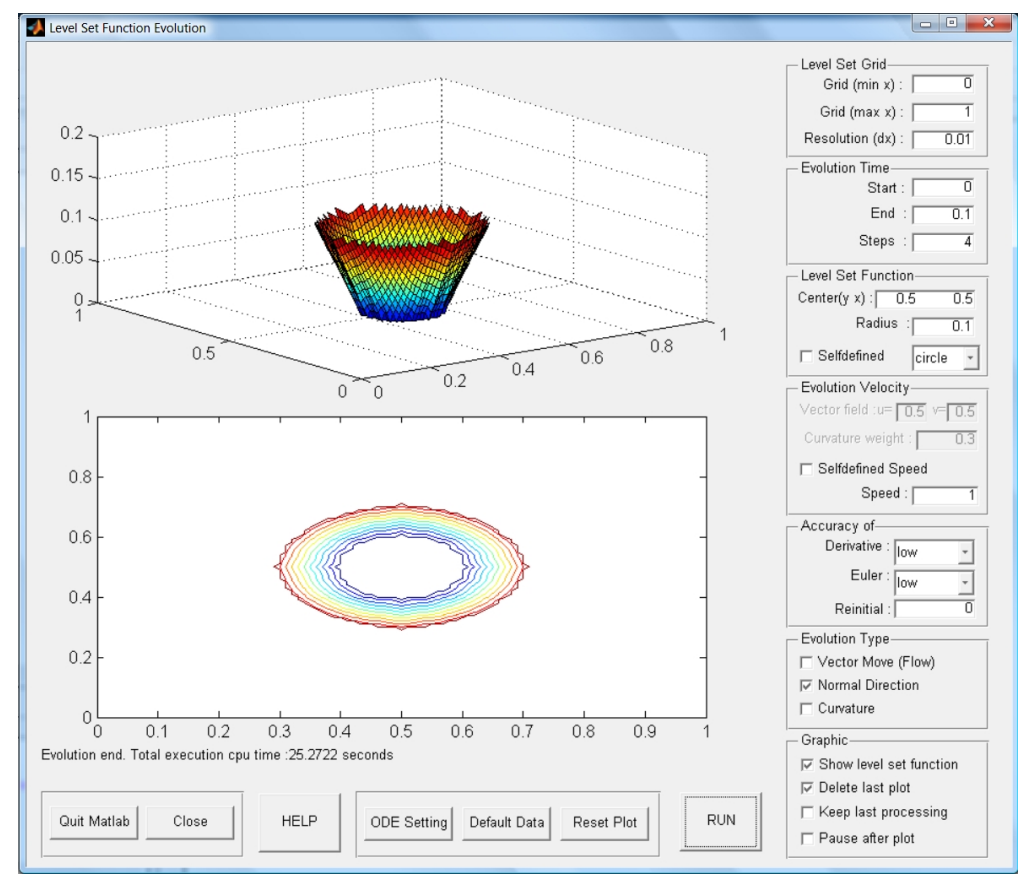

(b) Example for fast marching method

Figure 3.6: Sample results from the model - The model used same parameters run by the level set method and the fast marching method. 
3. METHODOLOGIES 


\section{4}

\section{Simulation and Numerical Analysis}

In the previous chapter, we discussed two simulation methods -the narrow band level set method and the fast marching method. In this chapter we use various scenarios to test and validate our simulation model based on these two methods. We assume multiple fuel types and fuel characteristics. The simulation model is tested on a number of homogeneous and heterogeneous hypothetical landscapes.

\subsection{Simulation Data and Equation}

Our simulations are performed on a $100 \times 100$ lattice consisting of identical square cells with parameters described in Tab. 4.1 for the evolution process. In some cases, we change the parameters. These cases are addressed individually for each scenario. Each cell contains the fire data describing its fuel data and elevation. The fuel data including fuel characteristics, fuel load, surface area to volume ratio, fuel bed depth, mean fuel energy content, moisture of extinction dead fuels are simplified to the 13 fuel models (Tab. 4.2). Elevation data are transfered from elevation raster map directly. Data values are assigned according to the implemented scenario, which ranged from the simplest case, a fire spreading on a homogeneous landscape on level terrain in the absence of wind, to fire spreading in the presence of a uniform wind, topography and with a heterogeneous fuel bed. The formula for ROS in the simulation model is Eq. 3.7) on page 21. The wind direction is controlled by a 2-dimensional $x y$ vector. 


\section{SIMULATION AND NUMERICAL ANALYSIS}

For example, south wind can be represented by [0 1$]$; west wind is $\left[\begin{array}{ll}1 & 0\end{array}\right]$; south west wind is [1 1 ]; south south west wind is [1 2], etc. The speed of the wind $U$ which is used in $W(U, \vartheta)$ of Eq. (3.7) could be converted to miles/hour if simulated on a real landscape. To simplify matters, the parameter $\beta$ of topography factor $\Phi_{s}$ is set to 1 in all of our scenarios.

Table 4.1: Fire simulation parameters I - default values used for different simulation scenarios.

\begin{tabular}{lr}
\hline Parameter & Value \\
\hline Domain & $\Omega=[-1,1] \times[-1,1]$ \\
Resolution & $\triangle x=\triangle y=0.01$ \\
Spatial discretization & 201 \\
Initial front & Circle \\
Circle center & $(0,0)$ \\
Initial radius & 0.25 \\
Time step & $\triangle t=0.1$ \\
\hline
\end{tabular}

The fuel data input to the Rothermel fire model is a set of numerical values that describe the fire behavior according to a fuel behavior model. There are 53 standard fire behavior fuel models (53FBFM) originating from the 13 fuel models (13FBFM) developed by Anderson [2] and further expanded to 40 additional fuel models by Scott and Burgan [29]. Natural fuels may be classified as one of the 53FBFM or 13FBFM depending on which fire behavior fuel model is chosen. To simplify matters in our model verification, values for the fuel load $\left(\mathrm{kg} \mathrm{m}^{-2}\right)$, fuel bed depth $(\mathrm{m})$, surface area to volume ratio of the fuel particles $\left(\mathrm{cm}^{-2} / \mathrm{cm}^{-3}\right)$, extinction moisture content and fuel heat content $\left(\mathrm{kJ} \mathrm{kg}^{-1}\right)$ are assembled into 13FBFM fuel models as shown in Tab. 4.2 . The rate of the fire spread through a uniform fuel array in the absence of wind and topography may be obtained by applying $\varepsilon_{0}$ to one of the $13 \mathrm{FBFM}$ fuel models.

For our simulations, we do not consider the combustibility index (i.e set $\xi=1$ ) except for Sec. 4.3.1. In that section, we test our model under two different types of fuel, $\alpha$ and $\beta$, and the amount of heat $H_{\alpha}$ produced from $\alpha$ increased along with the elapsed time until the total heat is reached to ignite fuel $\beta$. In one scenario the heat generated by $\alpha$ is not high enough to ignite fuel $\beta$ and the fire is extinguished. 
Table 4.2: Standard 13 fuel models - Parameters for the standard 13 fuel models developed by Anderson [2].

\begin{tabular}{|c|c|c|c|c|c|c|c|}
\hline \multirow{3}{*}{$\begin{array}{c}\text { Fuel } \\
\text { model }\end{array}$} & \multirow{3}{*}{ Typical fuel complex } & \multirow{2}{*}{\multicolumn{4}{|c|}{$\begin{array}{l}\text { Surface-to-volume ratio/fuel loading } \\
\qquad\left(\mathrm{ft}^{-1} / \mathrm{kg} \mathrm{ha}^{-1}\right)\end{array}$}} & \multirow{3}{*}{$\begin{array}{l}\text { Fuel bed } \\
\text { depth } \\
\text { (ft) }\end{array}$} & \multirow{3}{*}{$\begin{array}{r}\text { Moisture of } \\
\text { extinction } \\
\text { dead fuels } \\
(\%)\end{array}$} \\
\hline & & & & & & & \\
\hline & & $1 \mathrm{~h}$ & $10 \mathrm{~h}$ & $100 \mathrm{~h}$ & Live & & \\
\hline \multicolumn{8}{|c|}{ Grass and grass dominated models } \\
\hline 1 & Short grass $(<30 \mathrm{~cm})$ & 0.74 & 0.00 & 0.00 & 0.00 & 1.0 & 12 \\
\hline 2 & $\begin{array}{l}\text { Timber (grass and } \\
\text { understory) }\end{array}$ & 2.00 & 1.00 & 0.50 & 0.50 & 1.0 & 15 \\
\hline 3 & Tall grass $(75 \mathrm{~cm}+)$ & 3.01 & 0.00 & 0.00 & 0.00 & 2.5 & 25 \\
\hline \multicolumn{8}{|c|}{ Chaparral and shrub fields models } \\
\hline 4 & $\begin{array}{l}\text { High pocosin, } \\
\text { chaparral }(180 \mathrm{~cm}+)\end{array}$ & 5.01 & 4.01 & 2.00 & 5.01 & 6.0 & 20 \\
\hline 5 & Brush $(60 \mathrm{~cm})$ & 1.00 & 0.50 & 0.00 & 2.00 & 2.0 & 20 \\
\hline 6 & $\begin{array}{l}\text { Dormant brush, } \\
\text { hardwood slash }\end{array}$ & 1.50 & 2.50 & 2.00 & 0.00 & 2.5 & 25 \\
\hline 7 & $\begin{array}{l}\text { Southern rough, low } \\
\text { pocosin }(60-180 \mathrm{~cm})\end{array}$ & 1.13 & 1.87 & 1.50 & 0.37 & 2.5 & 40 \\
\hline \multicolumn{8}{|c|}{ Timber litter models } \\
\hline 8 & Closed timber litter & 1.50 & 1.00 & 2.50 & 0.00 & 0.2 & 30 \\
\hline 9 & Hardwood litter & 2.92 & 0.41 & 0.15 & 0.00 & 0.2 & 25 \\
\hline 10 & $\begin{array}{l}\text { Heavy timber litter } \\
\text { and understory }\end{array}$ & 3.01 & 2.00 & 5.01 & 2.00 & 1.0 & 25 \\
\hline \multicolumn{8}{|c|}{ Slash models } \\
\hline 11 & Light logging slash & 1.50 & 4.51 & 5.51 & 0.00 & 1.0 & 15 \\
\hline 12 & Medium logging slash & 4.01 & 14.03 & 16.53 & 0.00 & 2.3 & 20 \\
\hline 13 & Heavy logging slash & 7.01 & 23.04 & 28.05 & 0.00 & 3.0 & 25 \\
\hline
\end{tabular}

Heat content (heat of pre-ignition) $=8000 \mathrm{Btu} / \mathrm{lb}$ for all models. 


\subsection{Homogeneous Landscapes}

Fuel characteristics of short grass (Tab. 4.3 for samples were assigned to each cell for the scenarios involving homogeneous landscapes. The vegetation of the area is dominated by grass.

Table 4.3: Fuel model parameters - Standard fuel model parameters of short grass for samples.

\begin{tabular}{lc}
\hline Fuel Model & Parameters \\
\hline Fuel loads & \\
Fine dead fuel(1 h fuel load) & 0.74 tons $/ \mathrm{ac}$ \\
Medium dead fuel (10 h fuel load) & 0 tons $/ \mathrm{ac}$ \\
Live herbaceous fuel & 0 tons $/ \mathrm{ac}$ \\
Live woody fuel & 0 tons $/ \mathrm{ac}$ \\
Surface area to volume ratios & \\
Fine dead fuel(1 h timelag) & $3500 \mathrm{ft}^{2} / \mathrm{ft}^{3}$ \\
Live herbaceous fuel & $1500 \mathrm{ft}^{2} / \mathrm{ft}^{3}$ \\
Live woody fuel & $1500 \mathrm{ft}^{2} / \mathrm{ft}^{3}$ \\
Fuel bad depth & $1 \mathrm{ft}^{3}$ \\
Dead fuel moisture of extinction & $12 \%$ \\
Dead Fuel heat content & $8000 \mathrm{Btu}^{3} / \mathrm{b}$ \\
Live Fuel heat content & $8000 \mathrm{Btu} / \mathrm{lb}$ \\
\hline
\end{tabular}

ROS $=0.3 \mathrm{ch} / \mathrm{h} ;$ Fire length $=0.1 \mathrm{ft}$. at $11.8 \%$ of fuel moisture content.

\subsubsection{Case 1: No wind and zero slope}

In the absence of wind and on the flat terrain, plots of successive fire fronts should produce concentric circles (Byram [6], Finney [9], Luke and McArthur [18]). The simulation results are as expected and produce concentric circles. Results are presented in Fig. 4.1 .

\subsubsection{Case 2: Wind only}

With west wind, Fig. 4.2 shows the simulation results using the narrow band level set method and the fast marching method respectively. 


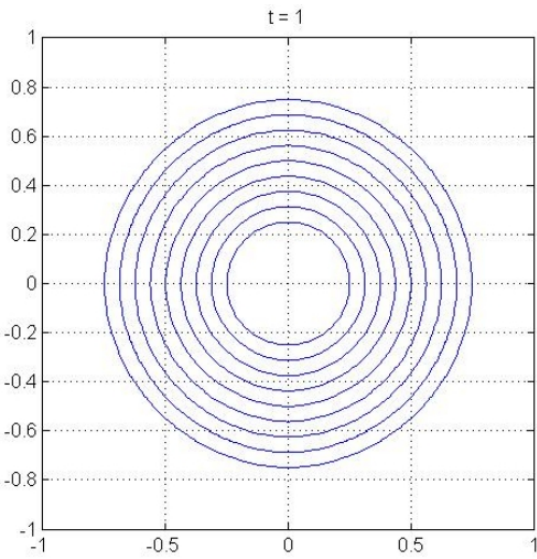

(a) A basic fire spread simulation using narrow band level set method

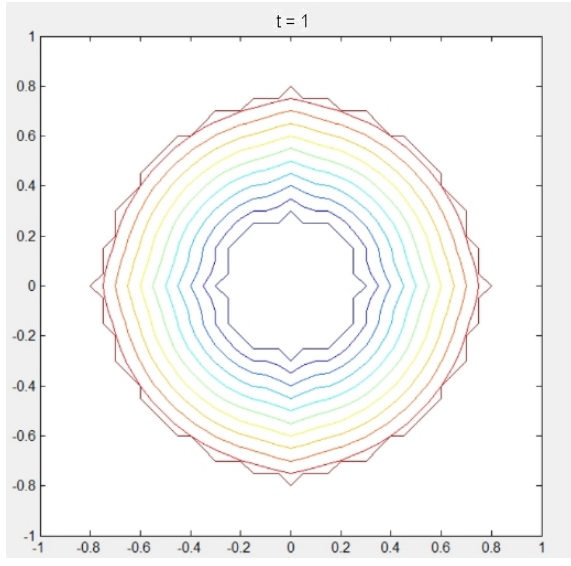

(b) A basic fire spread simulation using fast marching method

Figure 4.1: A basic fire simulated using two different methods - A basic fire spread simulation using narrow band level set method and fast marching method. The domain data is at Tab. 4.1 and fuel data is at Tab. 4.3

Table 4.4: Fire simulation parameters II - default values for different simulation scenarios.

\begin{tabular}{lclr}
\hline Parameter & Value & Parameter & Value \\
\hline$n$ & 3 & Domain & {$[0,3] \mathrm{x}[0,3]$} \\
$U$ & 100 & $t_{f}$ & 0.1 \\
$a$ & 0.5 & $\Delta t$ & 0.01 \\
$\varepsilon_{0}$ & 1.0 & Spatial & $N_{x}=N_{y}=101$ \\
$\alpha$ & 0.5 & Center & $(1.5,1.0)$ \\
$R O S$ & Eq. $(3.7)$ & Initial radius & 0.5 \\
\hline
\end{tabular}

\subsubsection{Case 3: Under wind and with slope}

Next we implement different simulations on a homogeneous landscape in the presence of south wind valued from $U=0$ to 50 at modified Rothermel's model (Eq. (3.7)) and at land with the slope to the east from 0 degree to 60 degrees using the narrow band level set method (Fig. 4.3) and the fast marching method (Fig. 4.4). The simulation 


\section{SIMULATION AND NUMERICAL ANALYSIS}

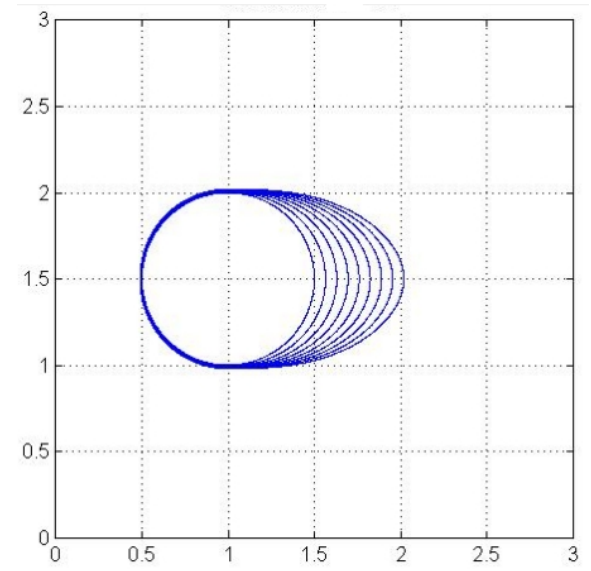

(a) A fire with west wind simulated using level set method

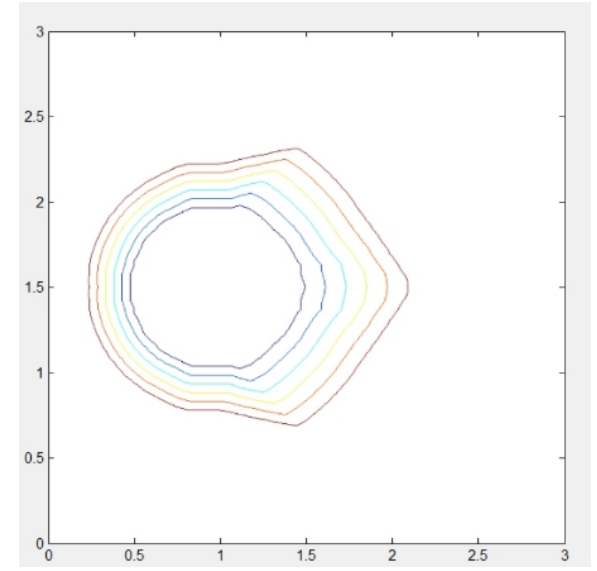

(b) A fire with west wind simulated using fast marching method

Figure 4.2: A fire with west wind simulated using two different methods - A fire spread under west wind simulation using level set method and a fast marching method. The domain data is at Tab. 4.4 and fuel data is at Tab. 4.3. Because the fast marching method considers only the adjacent neighboring cells as expanding directions, it might cause the distortions of the fire shape shown in Fig. $4.2 \mathrm{~b}$

ends after 4 steps starting at time period $[0,0.1]$ at the domain $[0,3] \times[0,3]$ with resolution $d x=d y=0.03$. Fire is ignited in the circle of radius 0.1 with center at $[1,1]$. The fuel factor $\varepsilon_{0}$ in Eq. (3.7) is set to 1 (on a Homogeneous fuel land).

Under the no wind and zero slope conditions, the shape of the fire is a circle (at bottom left corner of Fig. 4.3). Due to the impact of the wind, the shape of the fire front is transformed from a circle under no wind condition to an ellipse under wind condition. The degree of elongation depends on the speed of the wind. This is a commonly observed phenomenon in all simulation tests. When the speed of the wind increases from 0 to 50, the fire forms an elliptical shape which moves up (at the bottom line of Fig. 4.3). At the bottom right of Fig. 4.3 , the head fire goes up from $y=1.375$ to 2.75 on $y$-axis and the back fire goes from $y=0.75$ down to 0.625 on $y$-axis. The shape of successive fire fronts is expected as an approximate ellipse with the long-axis parallel to the direction of the wind (Byram [6], Finney [9], Luke and McArthur [18]). The short-axis of the ellipse is reduced when the speed of the wind increases. 
60

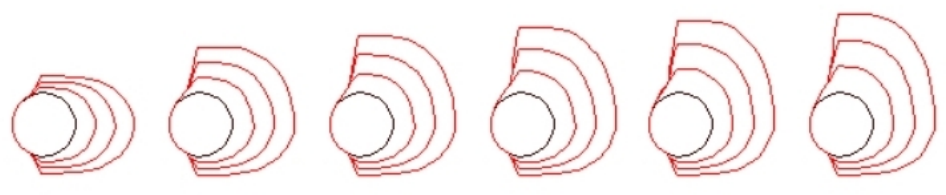

45

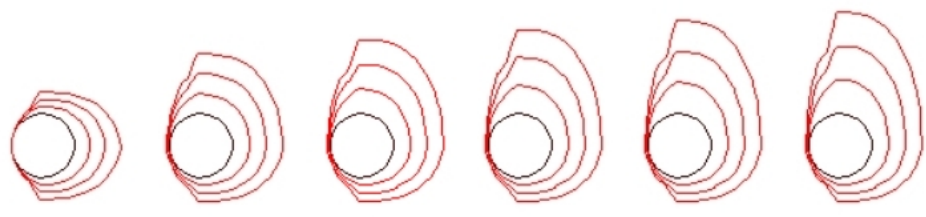

乞 $^{40 \bigcirc}$
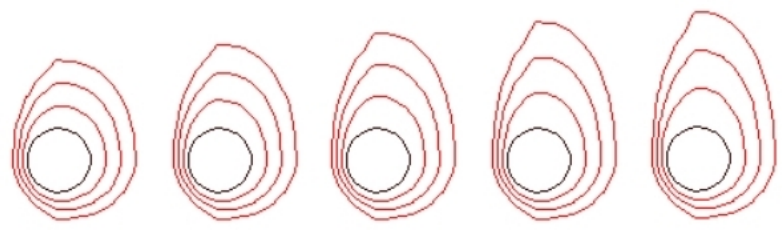

$\frac{\searrow}{\text { の }}$

30<smiles>c1ccc2ccccc2c1</smiles>
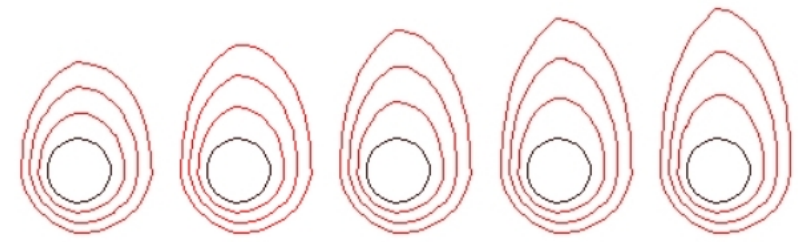

15<smiles>c1ccc2ccccc2c1</smiles><smiles>c1ccc2ccccc2c1</smiles>
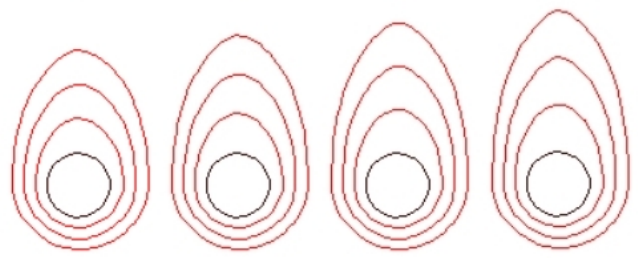

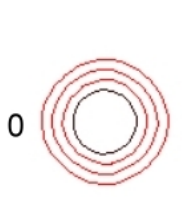

0

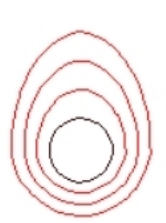

10

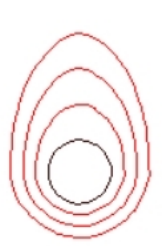

20

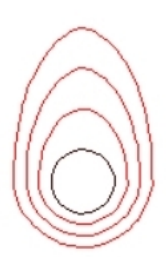

30

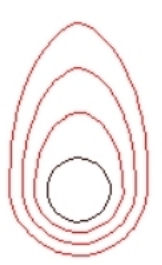

40

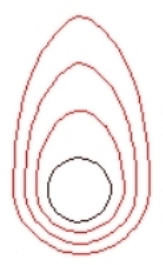

50

Wind Speed $(U)$

Figure 4.3: Surface fire shapes generated by the narrow band level set method - Surface fire shapes resulting from vectored cross-slope winds with west-facing slope and south winds. The slope results are horizontal projections. The parameters are at Tab. 4.4. and fuel data is not considered. 


\section{SIMULATION AND NUMERICAL ANALYSIS}

On the left vertical side of Fig. 4.3 and Fig. 4.4 the fire shape is changed along with the slope change. The slope effect is not obvious until the slope reaches $30^{\circ}$. Once the slope exceeds $60^{\circ}$, the fire does not move downward along with the slope change; just as the fire on a torch, it only moves up. This phenomenon is a specific reaction of the terrain factor part in any fire model which always uses $\tan ^{n}(\theta)$ ( $\operatorname{such}$ as in Equ 2.3) or $\exp (n \theta)$ (such as in Equ 2.4) as the factor in the fire spread formula, and $n$ is a parameter to control how much the fire is affected by the slope change. Because Rothermel's fire model was originally developed for flat terrain, the outputs from both simulation implementations are transformed from the surface plane (depending on the slope degree) back to the horizontal plane. Therefore, the fire shape is impacted less by the change of the slope than by change of the wind speed.

Fig. 4.5 shows a fire passing a hypothetical mountain on the north-east part of the landscape using the narrow band level set method. The mountain height is 0.5 units on a domain $[1,3] \times[1,3]$. The slope increases slowly on the lower part of the landscape, then increases fast in the middle part, and slow again near the mountain top. The fire spread is consistent with the change of the slope. The fire head moves forward faster after it passes the lower part of the mountain and moves slowly again near the top where it almost stops. On the downhill side, the fire spreads very slowly until the fire overlaps both sides of the hill.

Fig. 4.6 is the gradient map of Fig. 4.5a. Blue arrows show the magnitude and direction of gradient of each point on the landscape. Red line represents the fire front and red arrows are the norm of fire front. The gradient at each vertex on the fire front can be seen as $\tan \vartheta_{t}$. Apply scalar projection of gradient onto norm for each vertex on the fire front to get the next position for the vertex which is represented by red dot. If $\vec{a}$ is gradient, $\vec{b}$ is norm, and the angle between gradient and norm is $\theta$, the cosine of $\theta$ can be computed in terms of gradient and norm, by the following property of the dot product $\vec{a} \cdot \vec{b}$ :

$$
\cos \theta=\frac{\vec{a} \cdot \vec{b}}{|\vec{a}||\vec{b}|} .
$$

Once we get $\theta$, we can have the scalar projection of $\vec{a}$ on to norm by $\vec{a} \cos \theta$. 
60<smiles>c1ccc2ccccc2c1</smiles><smiles>C1=CC2C3CCC(c4ccccc43)C2C=C1</smiles>
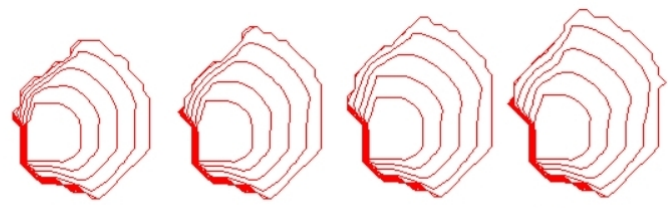

45<smiles>c1ccc2ccccc2c1</smiles><smiles></smiles><smiles></smiles><smiles></smiles>

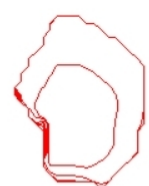<smiles></smiles>
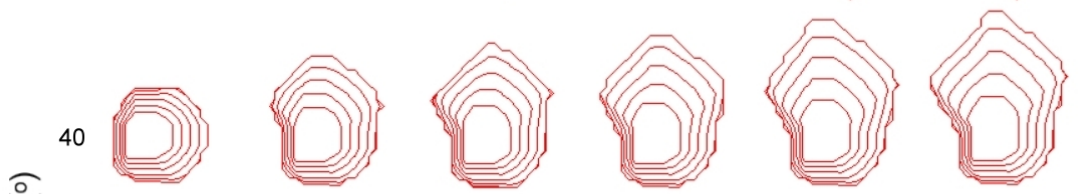

음

30
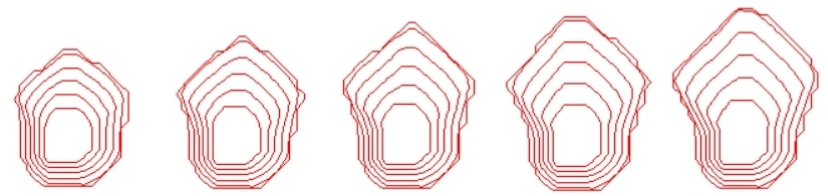

15
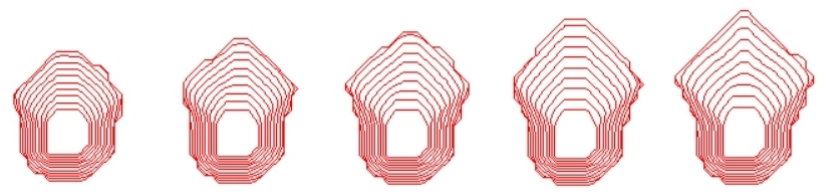

0
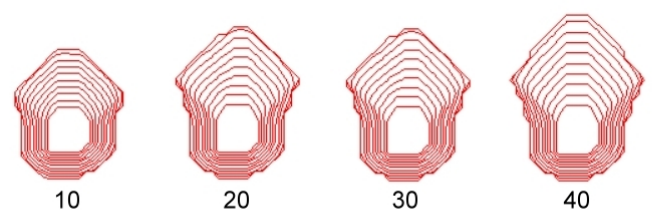

40

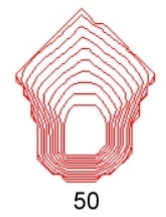

Wind Speed (U)

Figure 4.4: Surface fire shapes generated from the fast marching method Surface fire shapes resulting from vectored cross-slope winds with east-facing slope and south wind. The slope results are horizontal projections. The parameters are at Tab. 4.4 and fuel data will not be considered. 


\section{SIMULATION AND NUMERICAL ANALYSIS}

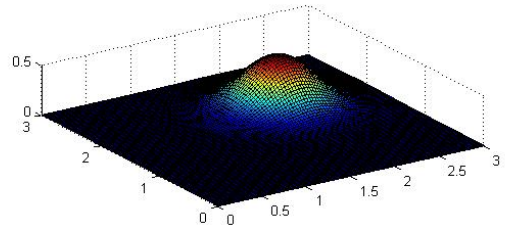

(a) A mountain in the north-east

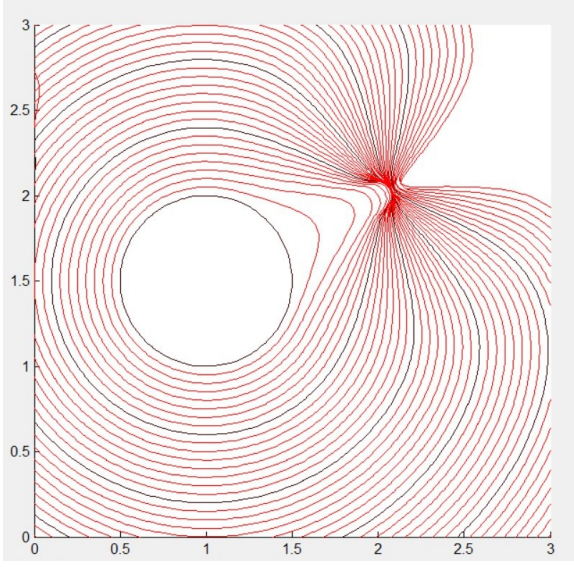

(b) Fire is passing a mountain

Figure 4.5: Fire passes a hill - Fire spread with homogeneous fuel, passes a mountain under no wind condition. The fire head moves faster from the lower part of the landscape to the upper part of the mountain and moves slowly near the top until it almost stops. This situation is consistent with the slope which evens out at the top. On the downhill side, the fire spreads very slowly and the fire from both hill sides overlaps at last. The parameters are at Tab. 4.4 .

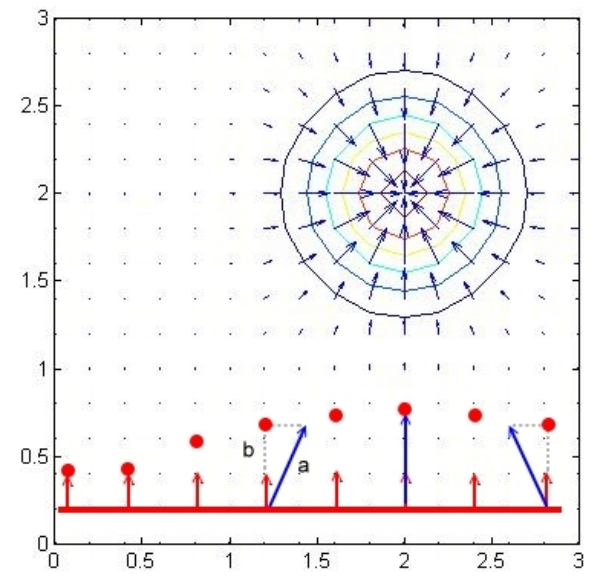

Figure 4.6: Slope $\tan \vartheta_{t}$ and fire front - The gradient map of Fig. 4.5a shows the magnitude and direction of gradient of each point on the landscape. Red line represents the fire front and red arrows are the norm of fire front. The gradient at each vertex on the fire front can be seen as $\tan \vartheta_{t}$. Apply scalar projection of gradient onto norm for each vertex on the fire front to get the next position for the vertex which is represented by red dot. 
Fig. 4.7 shows a fire passing a depression in the north-east part of the landscape using the narrow band level set method. The depression is on a domain $[1,3] \times[1,3]$ with the lowest height 0.4 units below zero. The fire spreads slowly around the depression but still moves forward driven by the west wind. On the uphill side of the depression, the fire spread picks up speed again. Both simulations of a fire passing a mountain and a depression generated the expected results.

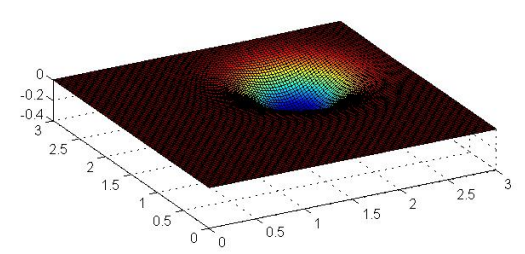

(a) A depression in the north-east

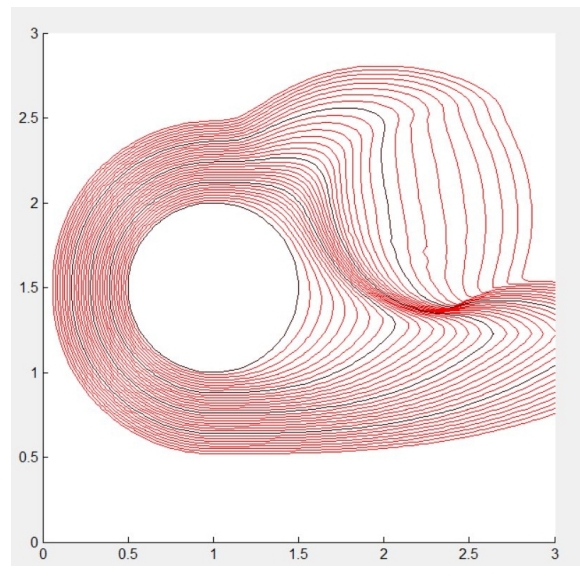

(b) Fire passes a depression under west wind

Figure 4.7: Fire passes a depression under west wind - Fire spread with homogeneous fuel, passing a depression under the west wind. The fire spreads slowly around the depressed side but still moves forward driven by the west wind. On the uphill side of the lowland, the fire spreads fast again. The parameters are at Tab. 4.4 .

\subsection{Heterogeneous Landscapes}

To incorporate the heterogeneous natural environment into the simulation, a complicated landscape model is introduced in this section. The simulation is implemented using the same parameter settings as in the previous section.

\subsubsection{Case 1: Two types of fuel}

In this section we simulate fire spread on a landscape with two different types of fuel with combustibility index which is defined in Eq. (3.8). A fire will spread from one fuel type to another fuel type if the heat captured by the unburned fuel ahead of the 


\section{SIMULATION AND NUMERICAL ANALYSIS}

fire front is sufficient to combust. Otherwise, the fire spread will stop. We use four matrices, $H_{t}$ the amount of heat released at time $t$ from a burning cell to its unburned neighboring cells; $H$ the heat required to ignite the fuel in the unburned cell; $H_{r}$ the heat released from the fuel of a burning cell for a period of time, and $H_{l}$ the total amount of heat a fuel can release to meet the combustibility index combined with the fuel model. When a fuel at cell $(i, j)$ is burning, it releases heat $H_{r}(i, j)$ and accumulates $H_{t}(k, l)$ at its neighboring cells as time progresses. When the total heat of $H_{t}(k, l)$ is greater than $H(k, l)$ the heat required to ignite cell $(k, l)$, the fire moves on to the neighboring cell. There is a limitation of $H_{l}(i, j)$ for the burning cell $(i, j)$ which is the maximum heat that can be released by the fuel at cell $(i, j)$. Once the heat released at a burning cell reaches its maximum while no neighboring cell is ignited, the fire stops at this cell.

Let us now assume that a fire is ignited at center $(1,1.5)$ with radius 0.3 on a landscape $([0,3] \times[0,3])$ where a grassland is on the left $(x<1.5)$ side and woods are on the right $(x>1.5)$. To simplify matters, the terrain and weather conditions will not be considered here. The parameters for this simulation are shown at Tab. 4.4 except fuel parameter $\varepsilon_{0}=1$ for grassland and $\varepsilon_{0}=2$ for woods. Fig. 4.8 shows the simulation results for a fire spread through a heterogeneous landscape. We set the heat which is required to ignite the woods from $H^{W}=1$ to $H^{W}=20$; the heat that could be released by the grassland is $H_{r}^{G}=1$; the maximum amount of heat that can be produced from grassland is $H_{l}^{G}=15$. Fig. $4.8 \mathrm{a}$ shows that Before $t<0.3$, the fire spreads through the grassland in a circular pattern at a steady speed. After $t=0.3$, the fire reaches the woods on the east side. Since the fire is sufficiently intense to ignite the woods, it continues to spread in this direction. The rate of spread(ROS), however, increases as the fire passes through the woods to approximately double its original speed. In all other directions, the rate of fire spread remains constant. For the remaining simulations we change the heat parameters $H^{W}$ of woods. In Fig. $4.8 \mathrm{~b}$, the released heat and required heat for woods are set to $H^{W}=5$ and the fire spread to the woods is held off until the required heat is reached. When we raise the heat required to ignite woods to $H^{W}=10$, Fig. $4.8 \mathrm{c}$ shows a pause line at $t=0.3$ but the fire still quickly passes the woods afterwards. Once we raise the required heat for woods ignition to $H^{W}=15$, there is an obvious stop line in Fig. $4.8 \mathrm{~d}$ at $t=0.3$ which the fire passes after $t=0.4$. If 


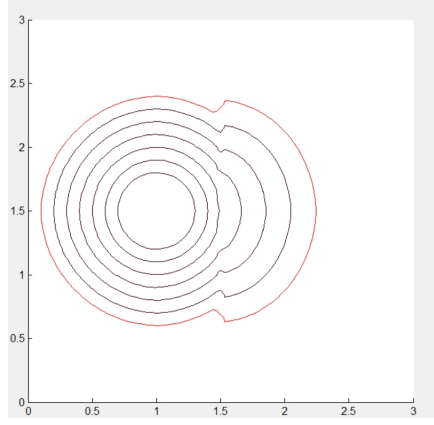

(a) $H^{W}=1 ;$ fire passes through different fuels without stopping.

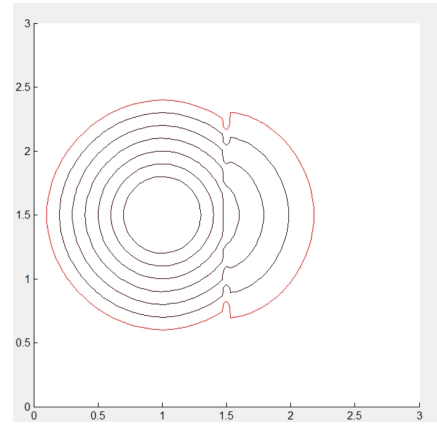

(c) $H^{W}=10$; fire pauses longer before moving on to another fuel.

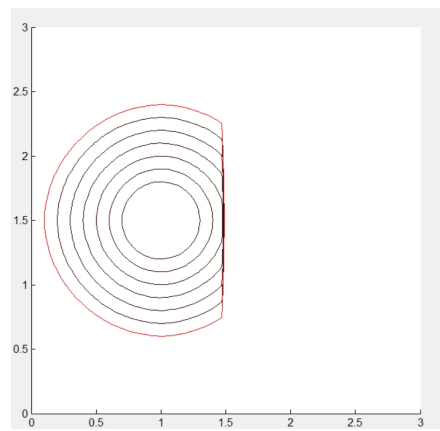

(e) When the total heat released by a fuel can't ignite the other fuel $\left(H_{l}^{G}<\right.$ $\left.H^{W}\right)$, the fire stops.

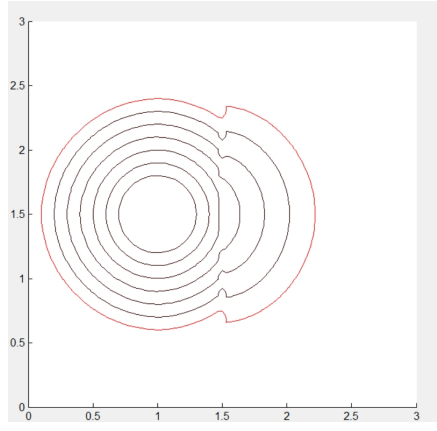

(b) $H^{W}=5$; fire pauses shortly to accumulate enough heat.

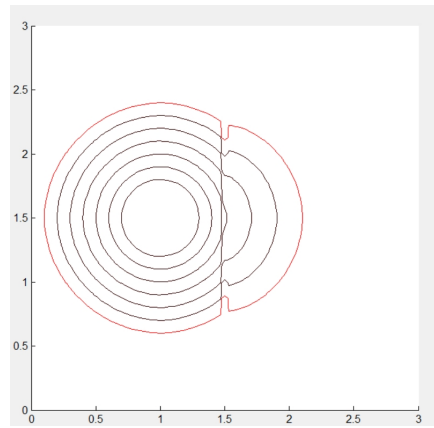

(d) $H^{W}=15$; A visible line is generated before the fire moves on.

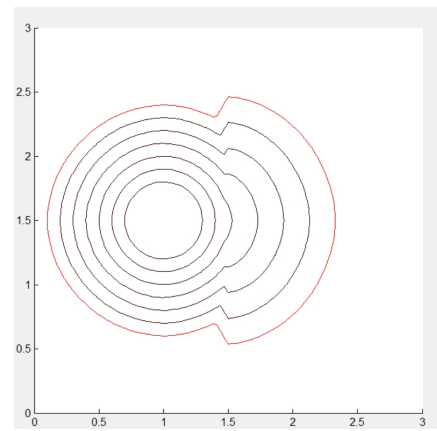

(f) A simulation without considering the heat convection between different fuels.

Figure 4.8: A fire expand at Heterogeneous fuel - Illustration of fire spread encountering different fuel types. $H^{W}$ is the heat required to ignite the fuel at $x>1.5 ; H_{r}^{G}$ is the heat released from the fuel at $x<1.5$ during a time period and the maximum amount of heat produced by this fuel is $H_{l}^{G}$. The graphics are set $H_{r}^{G}=1$ heat unit and $H_{l}^{G}=15$ heat units. The graph shows the fire spread under different $H^{W}$. The cumulation of $H_{t}^{W}$ must be bigger than $H^{W}$ to make the fire spread to the right side $(x>1.5)$; otherwise the fire will stop. 


\section{SIMULATION AND NUMERICAL ANALYSIS}

we keep raising the required heat for woods ignition to $H^{W}=20$, the fire stops before it reaches the woods, shown in Fig. $4.8 \mathrm{e}$, because the maximum heat of grassland fire is set to $H_{l}^{G}=15$ which is not high enough to ignite the woods.

These characteristics of fire spread between heterogeneous fuels is not considered in the vector based simulation model. Fig. $4.8 \mathrm{f}$ shows the simulation result from using a regular level set method without considering the heat convection between different fuels, where the fire never stops after it passes the woods.

\subsubsection{Case 2: Fuel only}

The landscape characteristics shown in Fig. 4.9a represent a leveled ground consisting of grassland (color coded in green) with a pond (color coded in blue) on the bottom left side of the landscape and a rock (color coded in brown) at the center right of the landscape. The pond is surrounded by wetland with gradually higher moisture content, and the fire spread speed is expected to be slow as the fire approaches the pond until it completely stops once it reaches the water. The fire is ignited at $(1,1.5)$ and is expanded from circle. From the fire simulation model (Fig. 4.9), we see that the south side of the landscape becomes wet, then the fire speed is decelerated and later the fire stops at the water's edge. The fire is unable to ignite the rock in the grassland while it continues to spread at a constant rate in all other directions and passes around the rock.

\subsubsection{Case 3: Complicated terrain and fuel type with wind}

A different scenario is simulated to determine the influence of the wind on the rate and pattern of the fire spread with heterogeneous fuel. The landscape consists of a pond on the west side and a small hill on the east side. This simulation is at domain $[-2,2] \times[-2,2]$ with resolution $d x=0.1$. Fire is ignited at $(0,0)$ and radius is 0.2 , with west wind originating. The parameters are at Tab. 4.4. At the beginning, the fire spreads toward the east with the wind and subsequently moves counterclockwise with changing wind directions. 


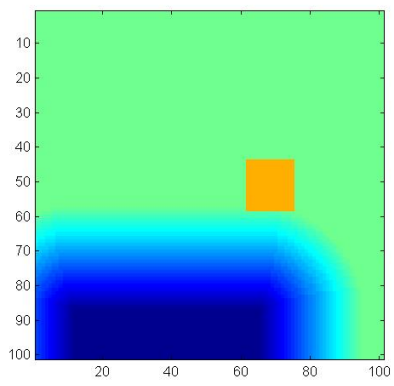

(a) Fuel map

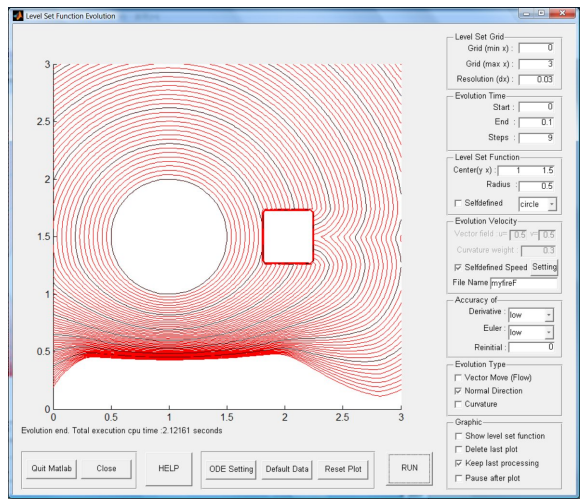

(c) slow speed when approaches the water

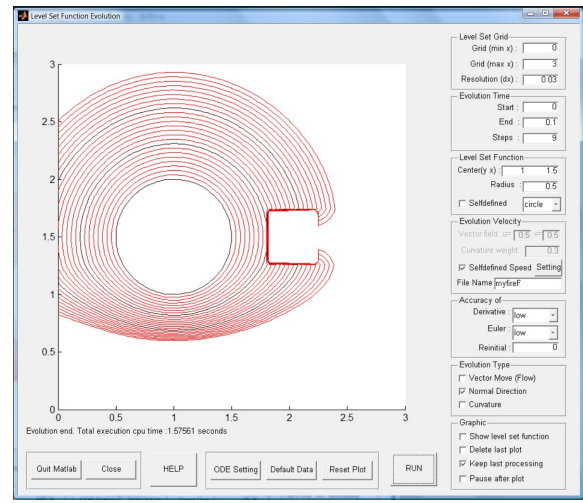

(b) pass around the rock

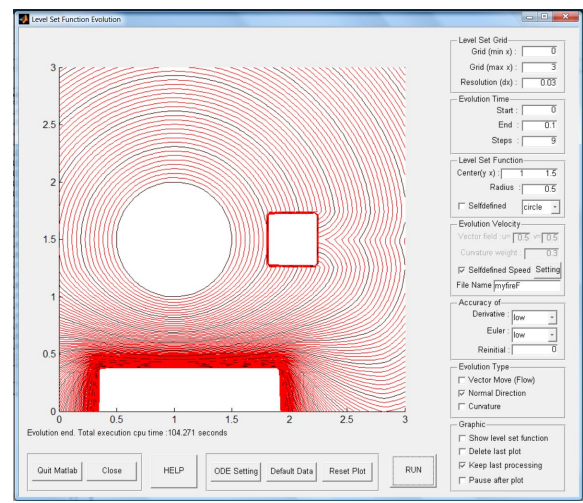

(d) stops when reaches the water

Figure 4.9: A fire with Heterogeneous fuel - Illustration of fire spread encountering different fuel types. $4.9 \mathrm{~b}$ showed the crossover easily to be resolved by the level set method. The parameters are at Tab. 4.4 


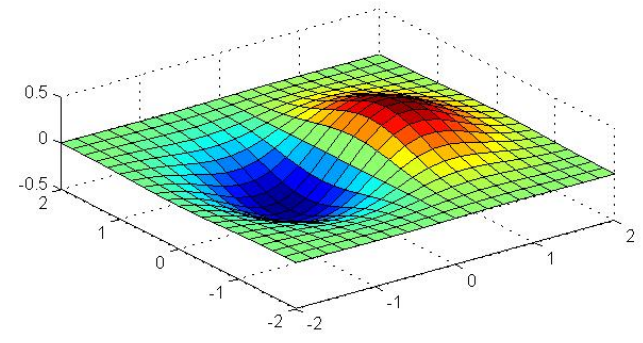

(a) A terrain with a pond on the west side (in blue) and a hill on the right side (in red).

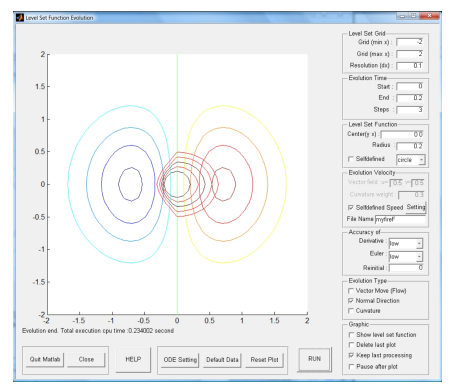

(b) West wind.

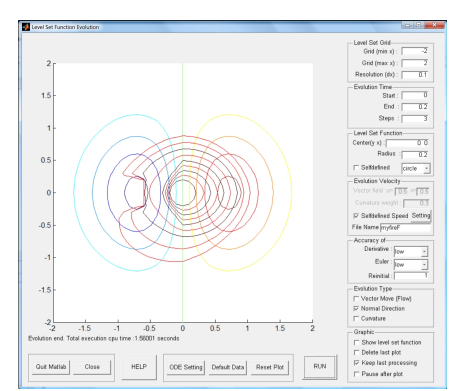

(d) North-east wind.

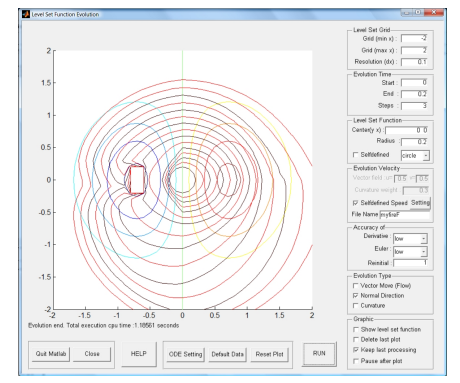

(f) North-west wind.

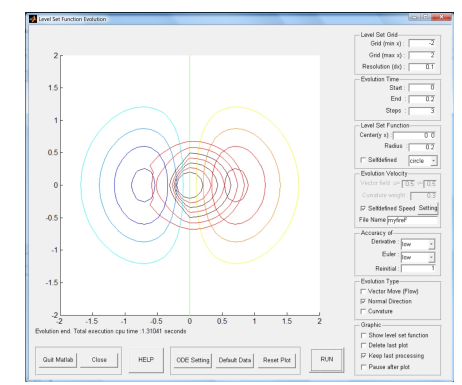

(c) East wind and double wind speed.

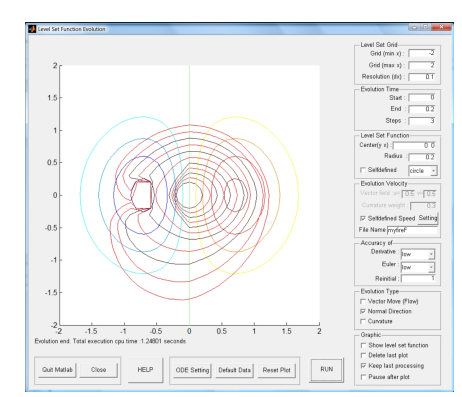

(e) North wind.

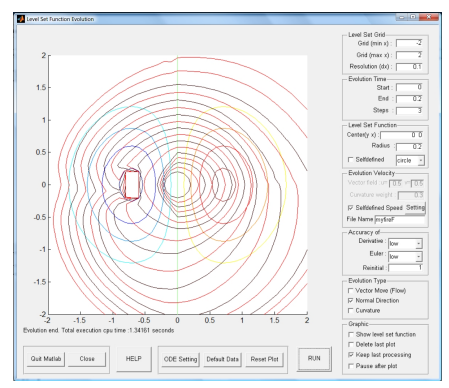

(g) West wind.

Figure 4.10: A fire simulated in a natural environment - A fire spreads on a terrain with a hill and a pond. Fire passes around pond with wind from different directions. 


\subsection{Numerical Accuracy Analysis}

In this section we perform an accuracy analysis of the implementation using narrow band level set method, and compare the error of the outcome under different resolutions. To test the accuracy of the numerical methods that we have implemented, we use the following relative error formula

$$
\operatorname{error}_{n}=\frac{\sum_{y} \sum_{x}\left|\varphi_{d_{n}}-\varphi_{d_{h}}\right|^{2}}{\sum_{y} \sum_{x}\left|\varphi_{d_{h}}\right|}
$$

where $\varphi_{d_{n}}$ is the numerical solution of a level set function $\varphi$ after $\Delta t$ evolution with the mesh grid size $d_{n}$ and $\varphi_{d_{h}}$ is the numerical solution with the smallest spatial mesh grid size, which we denote $d_{h}$, in our numerical simulations. Values of $\varphi$ on common points $(x, y)$ in different resolutions are used for calculating the error. We test only the first order of accuracy for the narrow band level set method. From the upwind scheme (3.15), we use

$$
\begin{aligned}
\phi_{+}^{\prime}\left(x_{0}\right) & \approx \frac{\phi\left(x_{0}+h\right)-\phi\left(x_{0}\right)}{h} \\
\phi_{-}^{\prime}\left(x_{0}\right) & \approx \frac{\phi\left(x_{0}\right)-\phi\left(x_{0}-h\right)}{h}
\end{aligned}
$$

to approximate $\phi^{\prime}(x)$ at $x_{0}$. For the right side of the upwind method, expand $\phi\left(x_{0}+h\right)$ by Taylor series

$$
\phi\left(x_{0}+h\right)=\phi\left(x_{0}\right)+h \phi^{\prime}\left(x_{0}\right)+(1 / 2) h^{2} \phi^{\prime \prime}\left(x_{0}\right)+(1 / 6) h^{3} \phi^{\prime \prime \prime}\left(x_{0}\right)+O\left(h^{4}\right)
$$

and combine this equation with Equ.(4.2) to

$$
\phi_{+}^{\prime}\left(x_{0}\right)-\phi^{\prime}\left(x_{0}\right)=(1 / 2) h \phi^{\prime \prime}\left(x_{0}\right)+(1 / 6) h^{2} \phi^{\prime \prime \prime}\left(x_{0}\right)+O\left(h^{3}\right) .
$$

Same to the left side of the upwind method, expand $\phi\left(x_{0}-h\right)$ by the Taylor series

$$
\phi\left(x_{0}-h\right)=\phi\left(x_{0}\right)-h \phi^{\prime}\left(x_{0}\right)+(1 / 2) h^{2} \phi^{\prime \prime}\left(x_{0}\right)-(1 / 6) h^{3} \phi^{\prime \prime \prime}\left(x_{0}\right)+O\left(h^{4}\right)
$$

and combine this equation with Equ.(4.3) to

$$
\phi_{-}^{\prime}\left(x_{0}\right)-\phi^{\prime}\left(x_{0}\right)=-(1 / 2) h \phi^{\prime \prime}\left(x_{0}\right)+(1 / 6) h^{2} \phi^{\prime \prime \prime}\left(x_{0}\right)+O\left(h^{3}\right) .
$$




\section{SIMULATION AND NUMERICAL ANALYSIS}

From the above (4.4) and (4.5), we obtain that the error is of order $O(h)$.

First, we test the simple fire spread from time 0 to time 0.1 with domain $[0,1] \times[0,1]$ using mesh grid size $d x=d y=\{0.1,0.05,0.025,0.0125,0.00625\}$ and calculate the error. The results are shown in Tab. 4.6 and Fig. 4.11. The execution time of the basic fire spread simulation and the grid size are in Tab. 4.5. For our computation, we use an AMD Athlon 642 dual-core processor with 3072MB system memory.

Table 4.5: Execution time for a basic fire - The execution time for different grid sizes to run the basic fire spread simulation.

\begin{tabular}{lrrrrr}
\hline$\triangle x$ & 0.1 & 0.05 & 0.025 & 0.0125 & 0.00625 \\
\hline Execution time (sec.) & 0.0521 & 0.0570 & 0.0671 & 0.1033 & 0.4255 \\
\hline
\end{tabular}

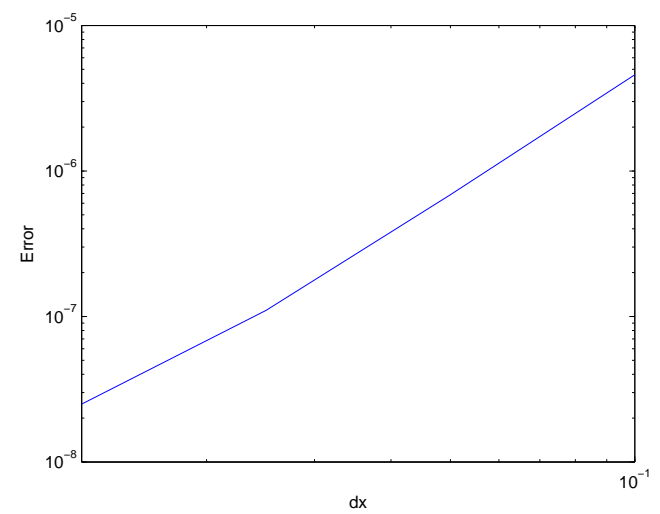

Figure 4.11: Error analysis of a basic fire - The figure shows that the higher the resolution, the smaller the relative error. (Data from Tab. 4.6.)

Table 4.6: Error analysis of a basic fire - The higher the resolution $\triangle x$ is, the smaller the $\operatorname{error}_{n}$ is.

\begin{tabular}{lrrrr}
\hline$\triangle x$ & 0.1 & 0.05 & 0.025 & 0.0125 \\
\hline error $\phi_{n}\left(\times 10^{-5}\right)$ & 0.4581 & 0.0687 & 0.0110 & 0.0025 \\
\hline
\end{tabular}

We continue our error analysis further with three different scenarios (fuel only; terrain only; wind, fuel and terrain) with mesh grid size $d x=d y=\{0.1,0.05,0.025,0.0125\}$ 
(see Tab. 4.7) for Troy fire which is used as a case study for the next chapter.

The fuel raster map consists of discrete numbers that cannot be interpolated while terrain changes can easily be interpolated. For example, if point $c$ is the middle point between point $a$ and point $b$, point $a$ 's elevation is 10 feet to the grassland and point $b$ is 20 feet to the woods, we may make point c's elevation to be 15 feet. At the same time, we can only select either grassland or woods for c's fuel. So when changing resolutions results in no new information gathered on a terrain map, the variation of terrain is rather stable and the high resolution will lead to higher accuracy. However if significant information is added or removed on a fuel map, it may lead to significant variations in results. This characteristic makes the variation of terrain more stable than that of fuel while resolution is changed. Fig. 4.13 shows the different resolutions for elevation, contour, and vegetation maps of Troy. The raster elevation map is on the left side; the contour map transformed from the raster elevation map is in the middle; and the vegetation (13FBFM fuel) map is on the right side. The resolution from top graphs to bottom graphs is $0.1,0.025,0.0125,0.003125$ respectively. The resolution of the raster map is 30 meter $\times 30$ meter. The elevation map is a continuous raster map and $13 \mathrm{FBFM}$ is a discrete raster map. Elevation maps and vegetation maps (13FBFM) are both downloaded from the LANDFIRE website [17.

Table 4.7: Resolution $\triangle x$ analysis - Comparison of the relative errors under fuel only; terrain only; wind, fuel and terrain scenario with different mesh sizes of $\triangle x$ between 0.1 and 0.00625 by Equ. 4.1 . The evolution time is between $[0,0.01]$

\begin{tabular}{lrrrr}
\hline$\triangle x$ & 0.1 & 0.05 & 0.025 & 0.0125 \\
\hline Fuel only & 0.0271 & 0.0307 & 0.0286 & 0.0001 \\
Terrain only & 0.4679 & 0.3203 & 0.2664 & 0.1901 \\
Wind, Fuel, \& Terrain & 0.0204 & 0.0404 & 0.0106 & 0.0016 \\
\hline values are in ten thousandths $\left(10^{-4}\right)$ & & & &
\end{tabular}

values are in ten thousandths $\left(10^{-4}\right)$.

Tab. 4.8 shows the execution time under different resolutions. Since it is normal to have some variation from one execution to the other in a multi-task OS, we use the average execution time of the first two runs as the execution time. The execution time varies at different resolutions. As expected, the higher the resolution, the more time it consumes. The execution time grows exponentially (Fig. 4.12). This result is also consistent with the level set computational complexity of $\bigcirc\left(n^{d}\right)$ both of time and 


\section{SIMULATION AND NUMERICAL ANALYSIS}

Table 4.8: Execution time at different resolutions - Comparison of the execution time(sec.) under fuel only; terrain only; wind, fuel and terrain with different resolutions of $\triangle x$ between 0.1 and 0.00625 during surface front evolution time period $[0,0.01]$.

\begin{tabular}{lrrrrr}
\hline$\triangle x$ & 0.1 & 0.05 & 0.025 & 0.0125 & 0.00625 \\
\hline Fuel only & 0.0087 & 0.0139 & 0.0168 & 0.0232 & 0.0858 \\
Terrain only & 0.0141 & 0.1156 & 0.2150 & 0.7323 & 0.8612 \\
Wind, Fuel, \& Terrain & 0.0171 & 0.0401 & 0.0739 & 0.1262 & 1.3854 \\
\hline
\end{tabular}

storage, where $n$ is the cross sectional for resolution of the spatial extents of the domain and $d$ is the number of spatial dimensions of the domain (Osher and Sethian [22]).

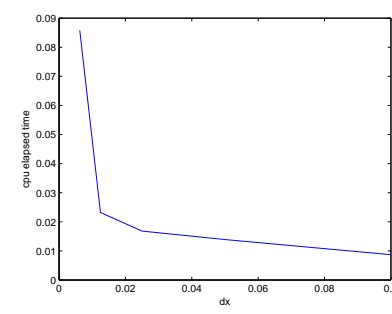

(a) Fuel only

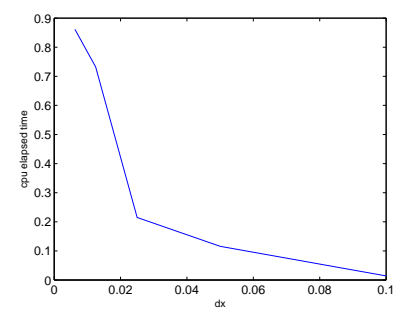

(b) Terrain only

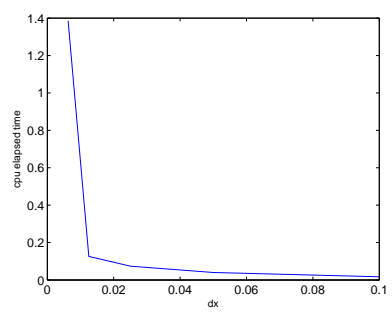

(c) Wind, fuel, and terrain

Figure 4.12: Execution time at different resolutions - The execution time under different resolutions. $\mathrm{X}$-axis is cpu elapsed time, and $\mathrm{y}$-axis is $d x$.

Tab. 4.8 shows the execution time under different resolutions. Since it is normal to have some variation from one execution to the other in a multi-task OS, we use the average execution time of the first two runs as the execution time. The execution time varies at different resolutions. As expected, the higher the resolution, the more time it consumes. The execution time grows exponentially (Fig. 4.12). This result is also consistent with the level set computational complexity of $\bigcirc\left(n^{d}\right)$ both of time and storage, where $n$ is the cross sectional for resolution of the spatial extents of the domain and $d$ is the number of spatial dimensions of the domain (Osher and Sethian [22]).

\subsubsection{Verification Conclusion}

The simulation model has been implemented with hypothetical landscapes under various assumed scenarios of fuel, wind and topography. Both the rate and pattern of fire 
spread predicted by the model are in close agreement with expectation and empirical evidence. However, there are limitations in the model using both narrow band level set method and fast marching method.

For the implementation using narrow band method, the distance function $\phi$ has the property that its magnitude $|\phi|$ is close to one at all times [30]. This is not always the case when we try to solve level set equations. This property makes it necessary to re-normalize to ensure that the system is reasonably stable around the interface. The normalization provides a process for the points, just next to the interface, to be reasonable values to keep the shape relatively smooth. It is time consuming to constantly re-normalize the distance functions and further risk distorting the shape of the interface.

For the implementation using fast marching method, more in-depth studies are needed. First, we only consider the adjacent neighboring cells and omit the diagonal neighboring cells. The omissions might cause the distortions of the fire shape especially under the condition of the wind. Fig. 4.2 shows the distortion of simulation from the fast marching method. Second, when simulation time is extended, the scale of the graphic will be automatically adjusted to be in alignment with the time axis during Matlab calculation. The automatically rescaled contour map may cause visual distortion and further lead to mis-prediction of the fire spread on the $x y$-plane. Third, the travel time is calculated as length of cell edge divided by fire spread speed $(a / R)$. Arithmetical operating errors will occur for a smaller $R$ especially when $R$ approaches zero. 


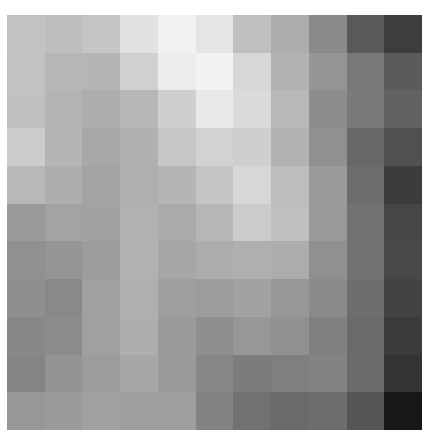

(a) elev. at size $11 \times 11$

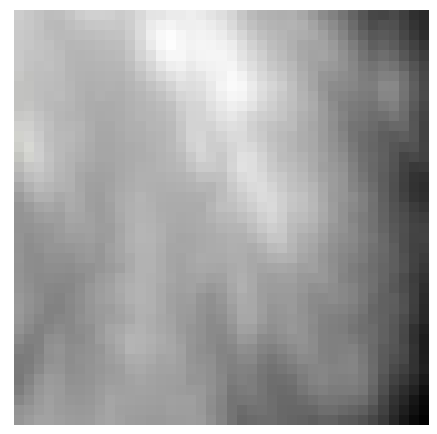

(d) elev. at size $41 \times 41$

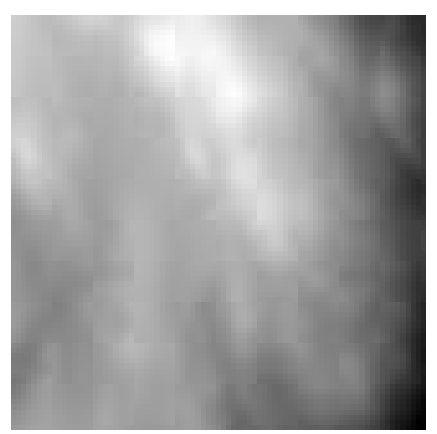

(g) elev. at size $81 \times 81$

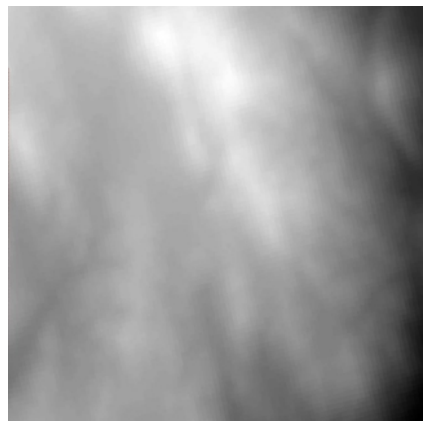

(j) elev. at size $321 \times 321$

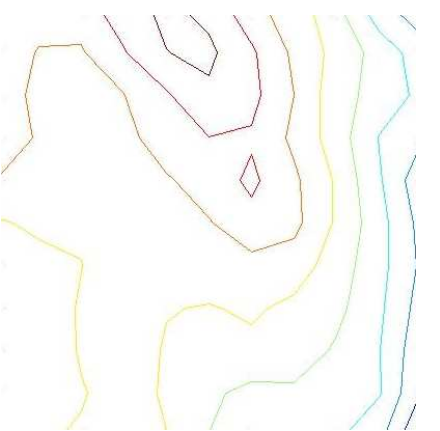

(b) Cont. from (a)

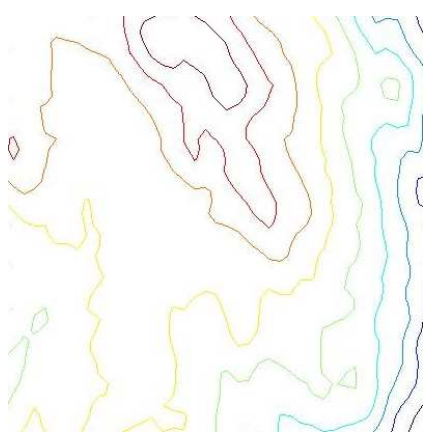

(e) Cont. from (d)

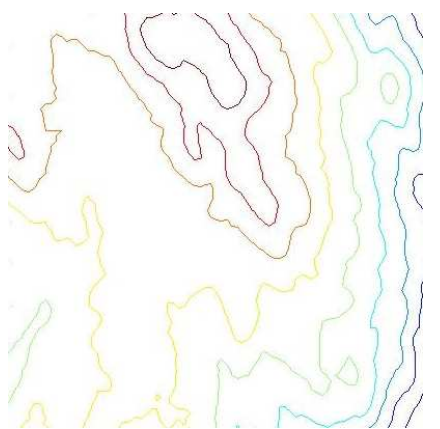

(h) Cont. from (g)

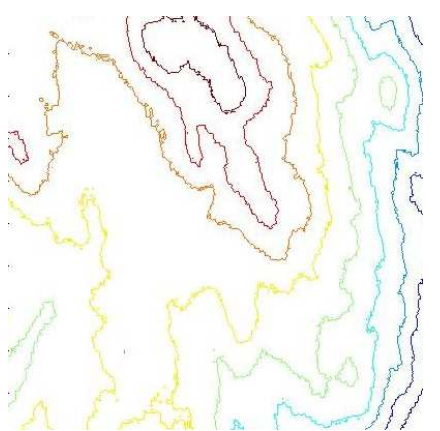

(k) Cont. from (j)

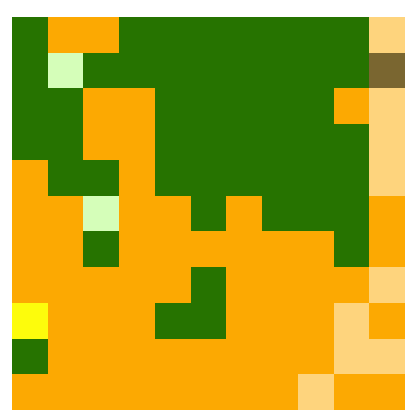

(c) veg. at size $11 \times 11$

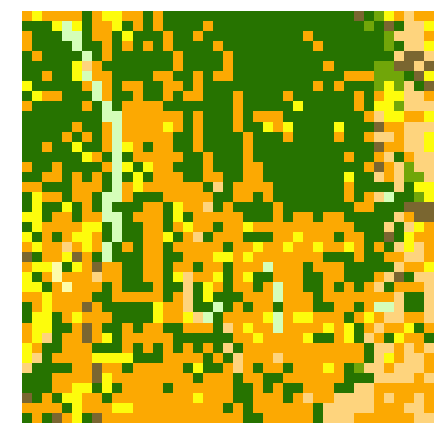

(f) veg. at size $41 \times 41$

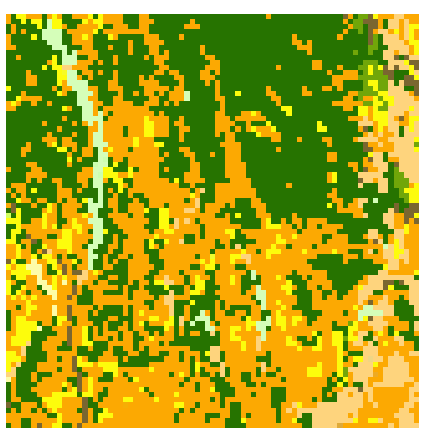

(i) veg. at size $81 \times 81$

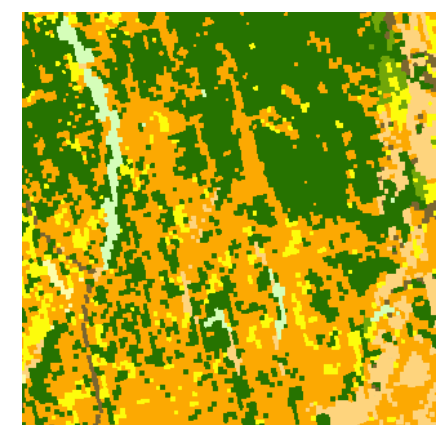

(1) veg. at size $321 \times 321$

Figure 4.13: Different resolution maps of Troy - Left side is raster maps of elevation; middle part is contour maps transform from left side; and vegetation map (13FBFM) is on the right side. The resolution from top to down is $0.1,0.025,0.0125,0.003125$ respectively. 
5

\section{Case Studies}

Two case studies are performed for our simulation model. One is called Troy fire, the other Colina fire. We choose the Troy fire because there are 20 aerial photos available that were taken from an aircraft between 13:29 and 15:55 on the day of the fire. Using these photos, we are able to examine the details of the actual fire spread. We choose the Colina fire because of the availability of the weather data for this fire. We download the terrain raster data and vegetation maps (13FBFM) from the US Forest Service, LANDFIRE website [17. We also use ArcGIS for the terrain data. ArcGIS [3] is a complete system for designing and managing solutions through the application of geographic knowledge.

The terrain raster data are direct inputs as data points of $\Phi_{s}$ in Eq. (3.7). The vegetation of the cropped area is mapped to the 13 fuel mod-

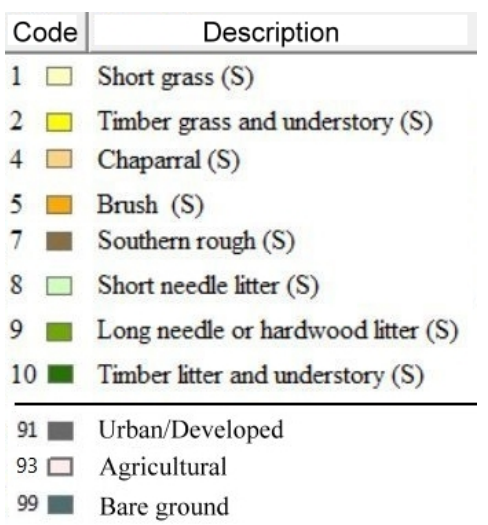

Figure 5.1: 13 FBFM color codes - Color codes of 13 FBFM in vegetation map els of Anderson [2]. Anderson divides his fuel model into 4 model groups: grass group(model 1 to 3 ), shrub group(model 4 to 7 ), timber group(model 8 to 10), and logging slash group(model 11 to 13). We extract 2001 vegetation map which is the closest available date to both our target fires. The fuel models are selected from the vegetation raster map and used as data points of $\varepsilon_{0}$ in Eq. (3.7). The color code description of fuel models we used in Troy fire and Colina 


\section{CASE STUDIES}

fire are shown at Fig. 5.1 .

We collect real fire images $5.6(\mathrm{~b})(\mathrm{d})(\mathrm{f})(\mathrm{h})(\mathrm{j}), 5.8 \mathrm{a}$, 5.14(b)(d)(f)(h)) for both fire areas from the Pacific Southwest Research Station (PSW) Airborne Sciences Aircraft. These images were disseminated in part by satellite communications in near-real time. The fire map measures the radiance of emitted thermal-infrared light, which readily penetrates the smoke. False-color images depict the apparent surface temperature (in Celsius), estimated from the radiance and a simple black-body model. Warmer tones represent recent or active combustion; areas of gray represent the cooling ash or warm bare ground. Low temperatures of unburned forest and cool ground are shown in green. The images are geographically referenced [32].

Table 5.1: Parameters of fuel model for case study - Parameters of fuel model from standard 13FBFM for case study.

\begin{tabular}{|c|c|c|c|c|c|c|c|c|}
\hline \multirow[t]{2}{*}{ Parameters } & \multicolumn{8}{|c|}{ 13FBFM Model Code } \\
\hline & 1 & 2 & 4 & 5 & 7 & 8 & 9 & 10 \\
\hline \multicolumn{9}{|l|}{ Fuel loads (tons/ac) } \\
\hline Fine dead fuel (1-h fuel load) & 0.74 & 2 & 5 & 1 & 1.1 & 1.5 & 2.9 & 3 \\
\hline Medium dead fuel (10-h fuel load ) & 0 & 1 & 4 & 0.5 & 1.9 & 1 & 0.41 & 2 \\
\hline Large dead fuel (100-h fuel load ) & 0 & 0.5 & 2 & 0 & 1.5 & 2.5 & 0.15 & 5 \\
\hline Live herbaceous fuel & 0 & 0.5 & 0 & 0 & 0 & 0 & 0 & 0 \\
\hline Live woody fuel & 0 & 0 & 5 & 2 & 0.37 & 0 & 0 & 2 \\
\hline \multicolumn{9}{|l|}{ Surface area to volume ratios $\left(\mathrm{ft}^{2} / \mathrm{ft}^{3}\right)$} \\
\hline Fine dead fuel & 3500 & 3000 & 2000 & 2000 & 1750 & 2000 & 2500 & 2000 \\
\hline Live herbaceous fuel & 1500 & 1500 & 1500 & 1500 & 1500 & 1500 & 1500 & 1500 \\
\hline Live woody fuel & 1500 & 1500 & 1500 & 1500 & 1500 & 1500 & 1500 & 1500 \\
\hline Fuel bed depth (feet) & 1 & 1 & 6 & 2 & 2.5 & 0.2 & 0.2 & 1 \\
\hline Dead fuel moisture of extinction & $12 \%$ & $15 \%$ & $20 \%$ & $20 \%$ & $40 \%$ & $30 \%$ & $25 \%$ & $25 \%$ \\
\hline Dead fuel heat content (Btu/lb) & 8000 & 8000 & 8000 & 8000 & 8000 & 8000 & 8000 & 8000 \\
\hline Live fuel heat content (Btu/lb) & 8000 & 8000 & 8000 & 8000 & 8000 & 8000 & 8000 & 8000 \\
\hline
\end{tabular}

Simulations are performed on a $43 \times 43$ matrix of identical square cells with a side length of 195 meters for Troy fire and 260 meters for Colina fire. Each cell contains local parameters describing its fuel data, elevation and wind data. Fuel data include fuel characteristics-fuel load, surface area to volume ratio, fuel bed depth, and moisture 
of extinction moisture content at Tab. 5.1, and moisture data at Fig 5.5. Elevation data are transfered from elevation raster map directly. Wind data can be inputted during the period of simulation.

\subsection{Case I : Troy Fire}

Troy is located in Mt. Laguna, two miles north of Thing Valley Road and Fred Canyon Road (Fig. 5.2).

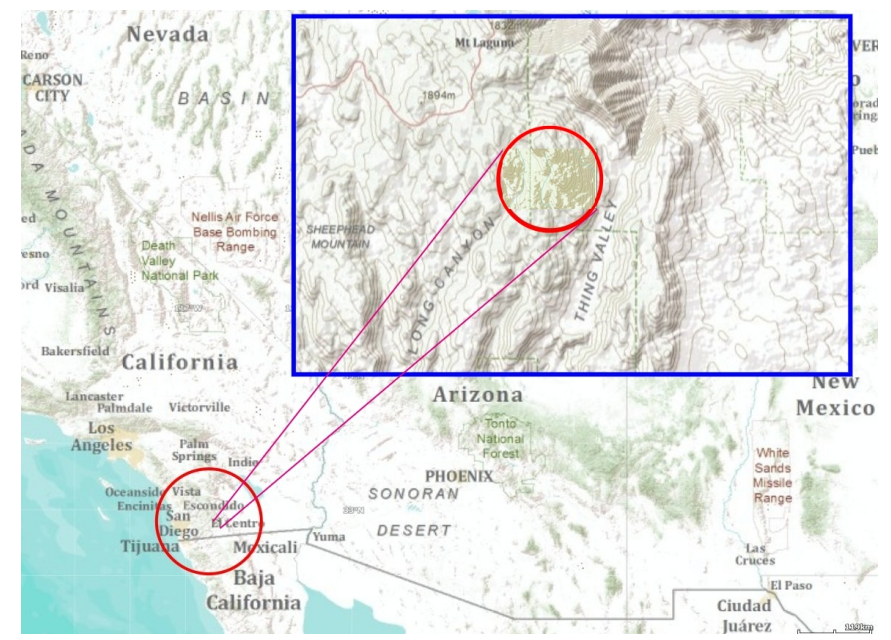

Figure 5.2: Map of Troy - The Map of Troy fire area

The Troy fire burned 1188 acres at Troy, San Diego County on June 19, 2002. PSW took a series of aerial photos using Airborne Sciences Aircraft. The images were disseminated by satellite communications in near-real time [32]. Fig. 5.3 shows the photos, cropped area and the time when the photos were taken.

We crop out the Troy fire area from ArcGIS map shown at Fig. 5.3 and divide it into a $43 \times 43$ matrix to create a fire area model. The contour map is transformed from raster data. Fig. 5.4 a shows $11290 \times 11290$ meter $^{2}$ of the Troy fire area. The vector contours for the majority of the Troy fire area are entered into a GIS at $50 \mathrm{~m}$ intervals and used to generate a $647 \times 647$ digital elevation model $(\mathrm{DEM})$ of the entire Troy fire area. The highest point is at an elevation of $450 \mathrm{~m}$ from the lowest point $(0 \mathrm{~m})$ in our 


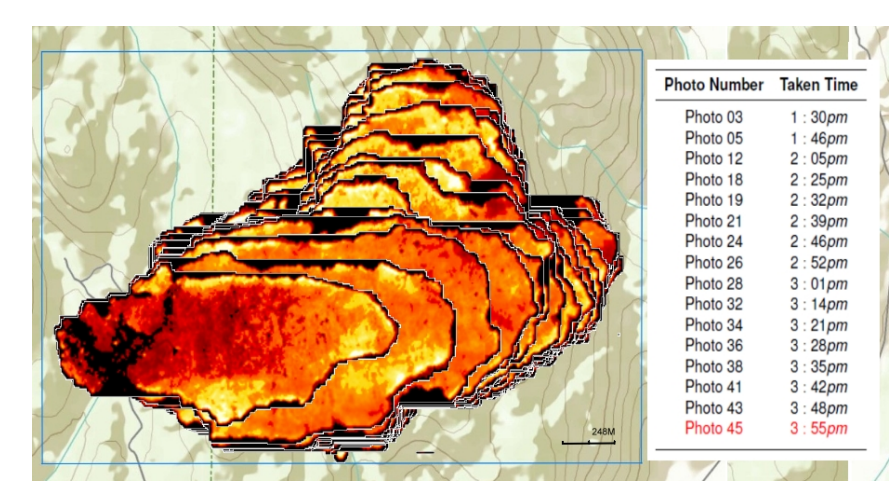

Figure 5.3: Troy fire and Time table - Tory fire photos and time of the photos taken. The area within the blue lined box is cropped from actual Troy fire ArcGIS map and divided to a $43 \times 43$ matrix for the simulation.

model.

The vegetation layers are mapped to Anderson's 13 fire behavior fuel models [2] from the LANDFIRE website [17]. The moisture data we assigned to each fuel model is shown at Fig. 5.5. The aerial images ( Fig. 5.4b) combined with ancillary GIS data layers improve the quality of the image classification. The ancillary GIS data layers include detailed vegetation coverage. The parameters for fuel are dominated by short grass, timber grass and under-story, chaparral and brush. The burned area is recorded using a global positioning system (GPS) and captured into GIS data layers.

\subsubsection{Narrow Band Level Set Method}

We use Eq.(3.7) in narrow band level set method to simulate the following scenarios: fire with vegetation only; with terrain only; with vegetation, terrain and west wind at three different wind speeds $-25 \mathrm{~m} / \mathrm{h}, 30 \mathrm{~m} / \mathrm{h}$ and $35 \mathrm{~m} / \mathrm{h}$. We check the results for the scenario with terrain only and notice that there is a valley on the east side of the area. The simulated fire did not pass the east side; however, the actual Troy fire kept going to the east side. Further examining the photos taken from the Troy fire, we notice that the fire spread quickly towards the east between 1:30 pm to 2:46 pm although at that time the fire was on a down slope towards the east. The vegetation map showed no significant changes. The fire spread might have been driven by the wind on the 


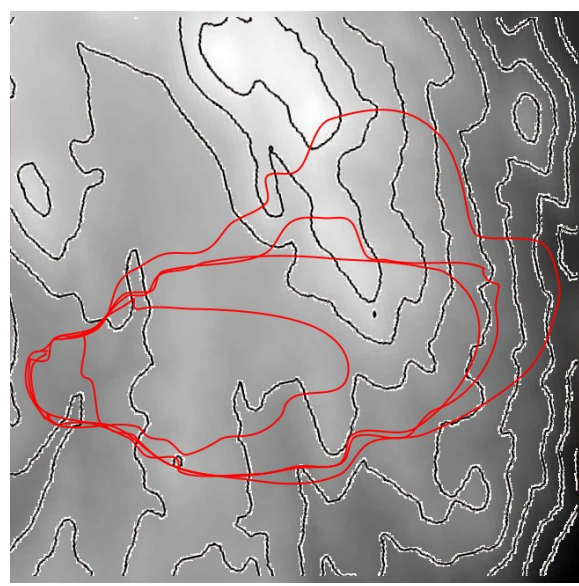

(a) The terrain contour map of the Troy fire area. The darker the color, the lower the elevation.

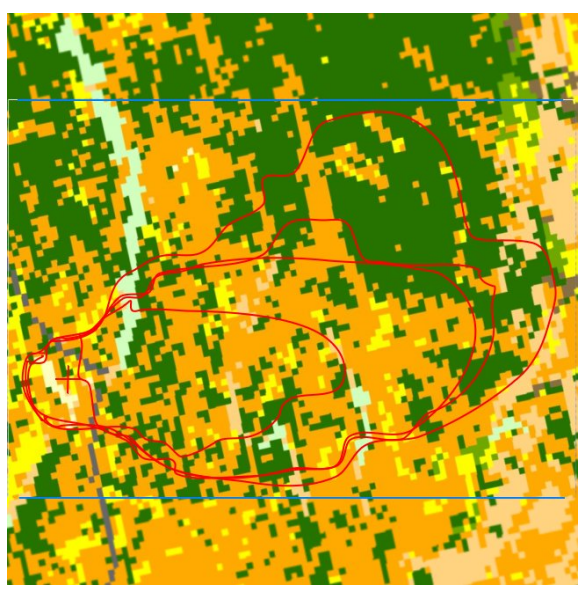

(b) The 13 FBFM vegetation map of the Troy fire area. The green part represents timber and the brown part represents shrub. The details of color code is shown in Fig. 5.1

Figure 5.4: Terrain and vegetation maps of Troy - Contour map and vegetation map of the Troy fire area. The contours are transformed from an elevation raster map. The raster data plotted on those two maps are used as data points of $\Phi_{s}$ and $\varepsilon_{0}$ in Eq. (3.9) to calculate the fire spread. Both elevation and vegetation raster maps are provided by LANDFIRE website [17. Red lines represent the fire front expansion.

\begin{tabular}{lccc}
\multicolumn{4}{c}{ Moisture Data Assigned to Fuel Model } \\
\cline { 2 - 5 } & \multicolumn{4}{c}{ Moisture(\%) } \\
\cline { 2 - 5 } Fuel Model & $1 \mathrm{~h}$ & $10 \mathrm{~h}$ & 100h Her or woody \\
\hline 1 short grass & 1 & & 300 \\
\hline 2 timber grass and understory & 15 & 1 & 100 \\
\hline 4 chaparral & 20 & 1 & 30 \\
\hline 5 brush & 17 & 1 & 300 \\
\hline 7 southern rough & 1 & 1 & \\
\hline 9 long needle or hardwood litter & 1 & 1 & \\
\hline 10 timber litter and understory & 1 & 1 & \\
\hline \hline
\end{tabular}

Figure 5.5: Moisture data table for fuel models - The moisture data assigned to each fuel models for Tory fire and Colina fire simulation are used in case studies. 


\section{CASE STUDIES}

downward slope with no changes of vegetation. Therefore, we conclude there is a west wind present at that time. We compare the results under three different wind speeds and find that the simulation results under wind speed of $30 \mathrm{~m} / \mathrm{h}$ is the closest to the Troy fire results. Therefore, we will assume wind speed of $30 \mathrm{~m} / \mathrm{h}$ throughout the rest of the simulation scenarios. Fig. 5.6 shows the simulated Troy fire with a west wind of $30 \mathrm{mph}$. Another scenario occurs at 2:52 pm. The fire spread accelerates towards the north, while still being on the downward slope. With no or little wind, the fire is expected to slow down when it is on a downward slope. Hence there must be a different factor that drives the fire forward. Further examination reveals the fuel type changes from grass to wood at this time. Therefore, the fuel type is likely the cause for the fire spread picking up speed. Later, we also run the fuel only and homogeneous vegetation simulation, and the results are consistent with our observations.

In order to compare the simulation results to the actual burned area, we color code the simulated burned areas as red and the actual burned areas as green. We then analyze the overlap. The comparison of the boundary after two and a half hours of fire (Fig. 5.7) reveals that $92 \%$ of the actual burned area is captured by our simulation. The simulated burned area included an area of $8 \%$ that is not burned at Troy (Overestimate) and $10 \%$ is burned at Troy but is not burned in our simulation (Underestimate).

Airborne Sciences Aircraft, starting from 13:29:53 to 15:55:43 took 45 photos of the fire. There was a prolonged break after photo 45 . The last photo was taken 2 hours 35 minutes later at 18:30 pm. If we continue the simulation run, it will result in Fig. 5.8b, closely matching the aerial photograph taken at 18:30:45 (Fig. 5.8a).

\subsubsection{Fast Marching Method}

The fast marching method is to calculate the travel time of fire spread to adjacent unburned cells from burning cells and to update the status of cells that reflected the shortest fire traveling time. We assume $a$ to be the side length of the cell and $R$ to be the fire spread model Eq. (3.7). By calculating the fire traveling time $(a / R)$, we will have a data set of travel time and could update the status of the unburned cells based on the shortest travel time of this data set. One limitation is that the possible next 


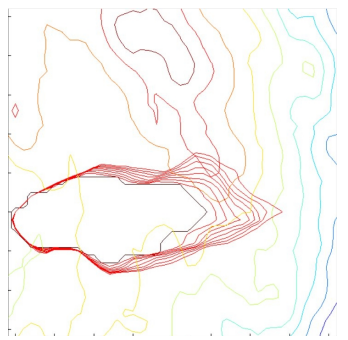

(a) Simu. at 14:00

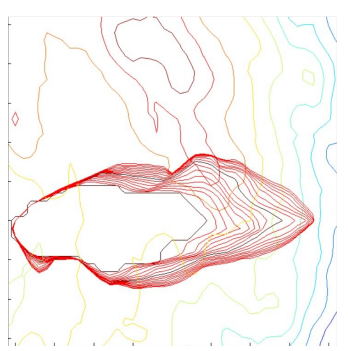

(c) Simu. at 14:30

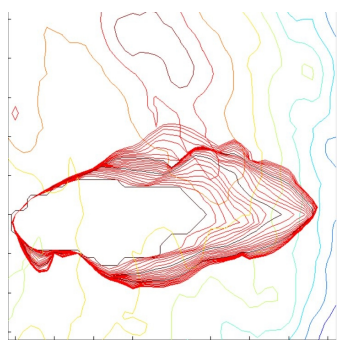

(e) Simu. at 15:00

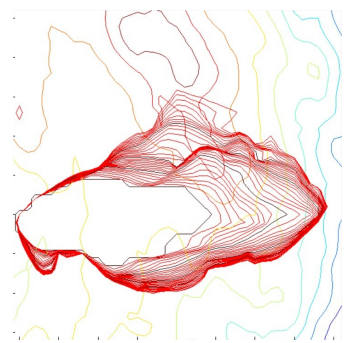

(g) Simu. at 15:30

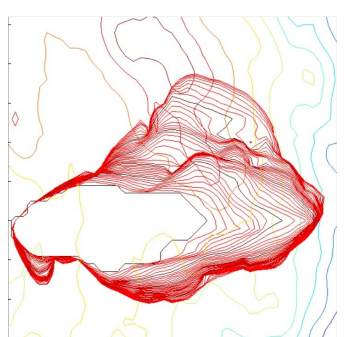

(i) Simu. at 16:00

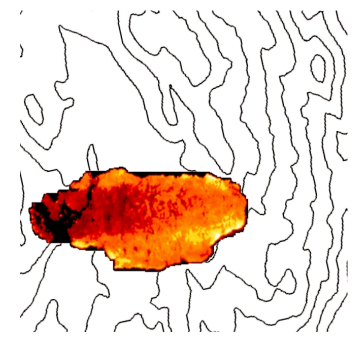

(b) Photo at 14:05

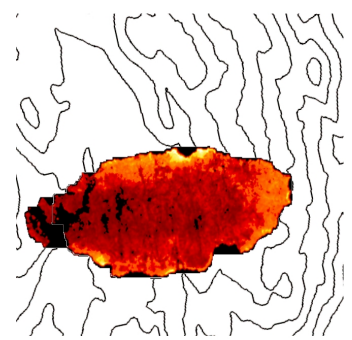

(d) Photo at 14:39

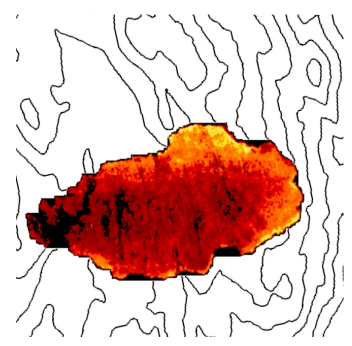

(f) Photo at 15:01

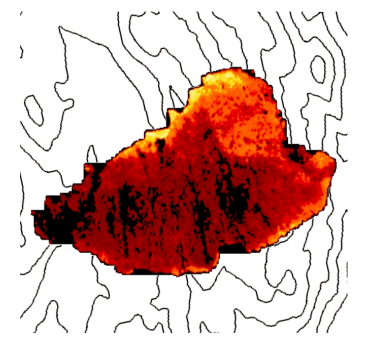

(h) Photo at 15:28

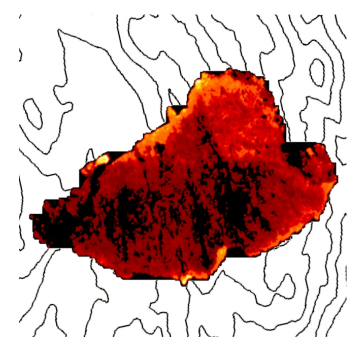

(j) Photo at 15:55

Figure 5.6: Simulated Troy fire - Simulated Troy fire using narrow band method compared with the photos of the real fire. 


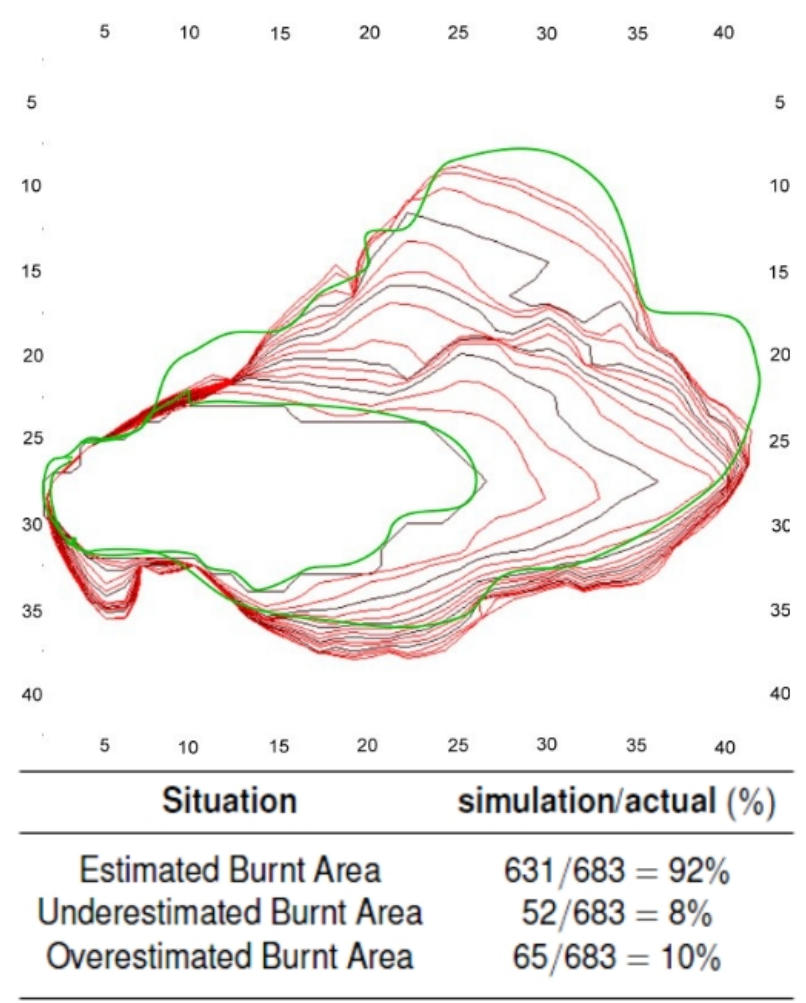

Figure 5.7: Troy fire comparison measure - Fire boundary comparison after two and half hours of burning. The green boundary represents actual burned areas and the red boundary represents the simulated burned areas. $92 \%$ of the real burned areas is overlapped with the simulated areas, $10 \%$ of burned is overestimated, and $8 \%$ is underestimated.

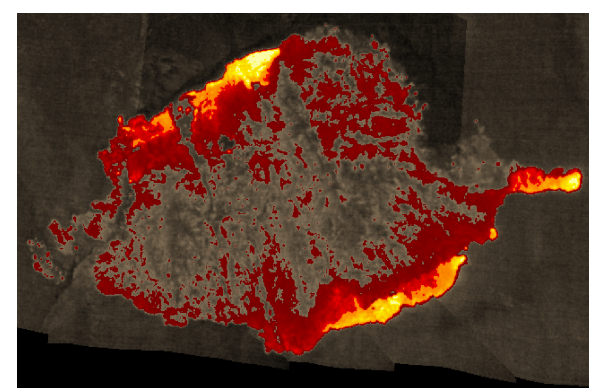

(a) The final photo was taken at 18:30.

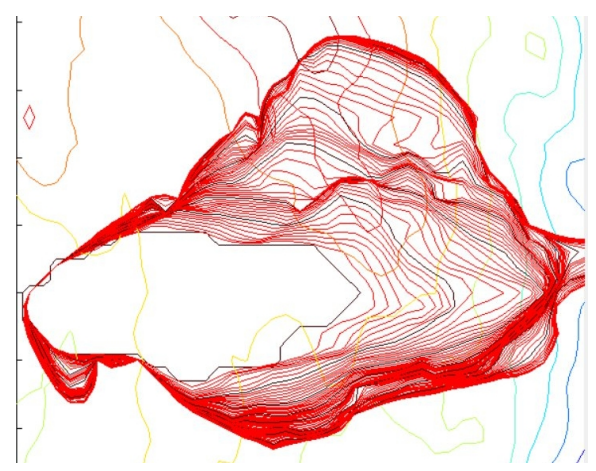

(b) The simulation continues

Figure 5.8: Comparison of the final photo with the simulation - The final photo taken at 18:30 pm compared to our simulation output. 
ignited cell is being considered among only the adjacent neighboring cells of a burning cell but not the diagonal cells. The limitation of fire spread angles might cause the less smooth fire shape and less precise result than those simulated using the narrow band level set method. The result of simulation of the Troy fire using fast marching method is in Fig. 5.9

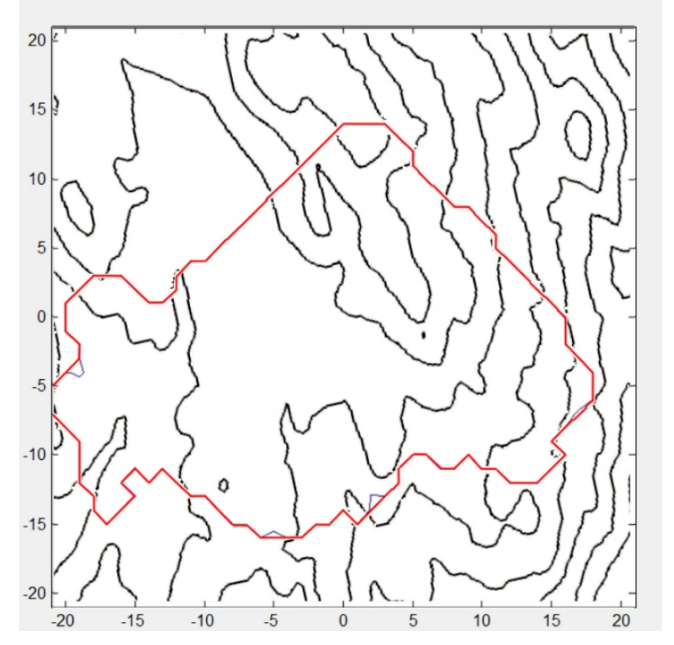

Figure 5.9: Troy fire simulation using the fast marching method - Troy fire simulation using the fast marching method. The fact that spread angles are limited in fast marching method makes the fire shape to be unnaturally angular.

In order to compare the simulation results to the actual burned area, we color code the simulated burned area as green and the actual burned area as black. Then we compare the results. A boundary comparison (Fig. 5.10) of the actual burned area to the simulated burned area after two and a half hours fire reveals $89 \%$ of overlapping burned area. The simulated burned area includes $22 \%$ of land that was not burned at Troy (Overestimate) and $11 \%$ of land that was burned but is not captured by our simulation (Underestimate).

\subsection{Case II : Colina Fire}

The Colina fire occurred on September 10, 2007 in Riverside County, CA. (Fig. 5.11) PSW Airborne Sciences Aircraft collected images of the fire and disseminated them 


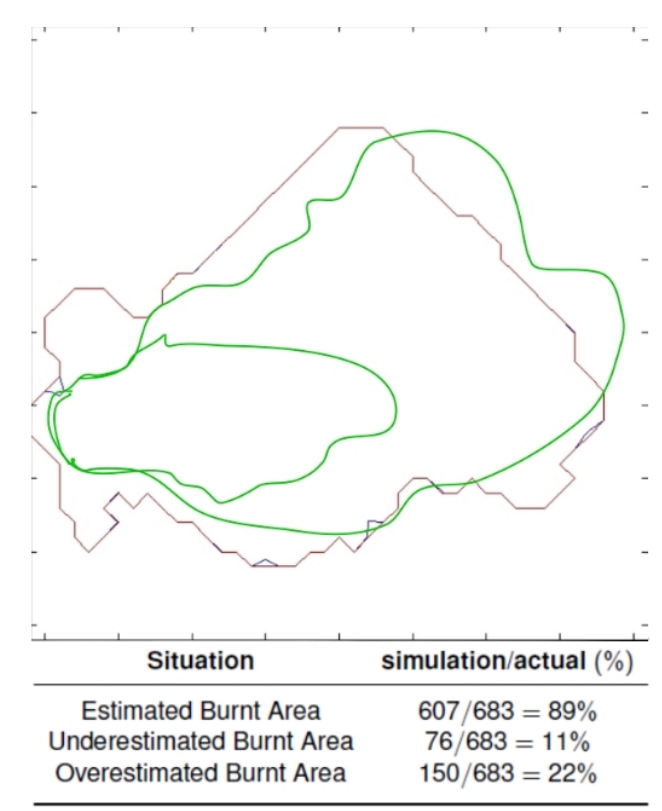

Figure 5.10: Troy fire simulation comparison using the fast marching method - Boundary comparison after two and half hours fire. The green boundary is the actual burned area, and the black boundary is the simulated burned area. $89 \%$ of the real burned area overlapped with the simulated burned area, $22 \%$ is overestimated burned areas, and $11 \%$ is underestimated burned areas.

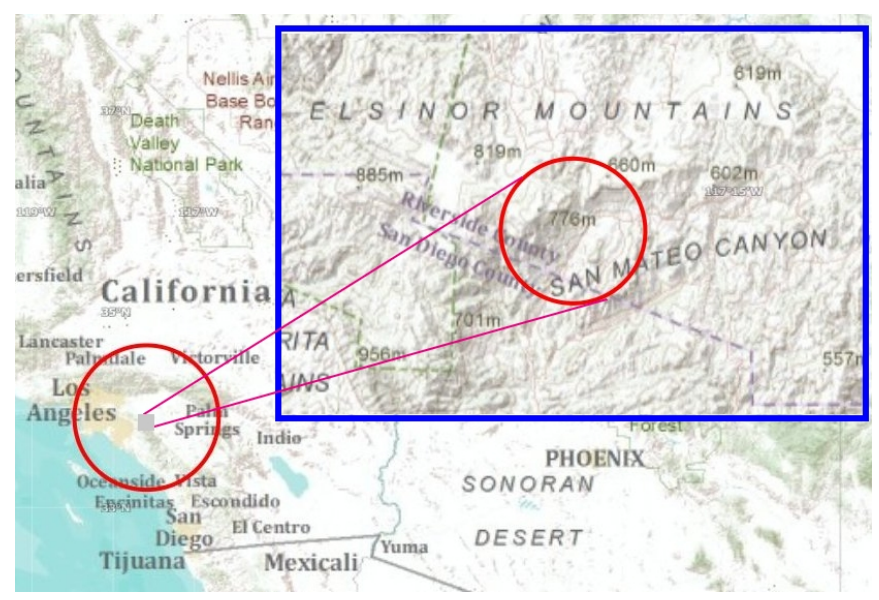

Figure 5.11: Map of Colina - The area of the Colina fire 
in part by using satellite communications in near-real time. The aerial photos were taken at 15:36, 17:50, 18:52, and at 20:06. Fig. 5.12 shows the fire map and weather conditions. The timing of the weather condition information does not match exactly the timing of the photographs.

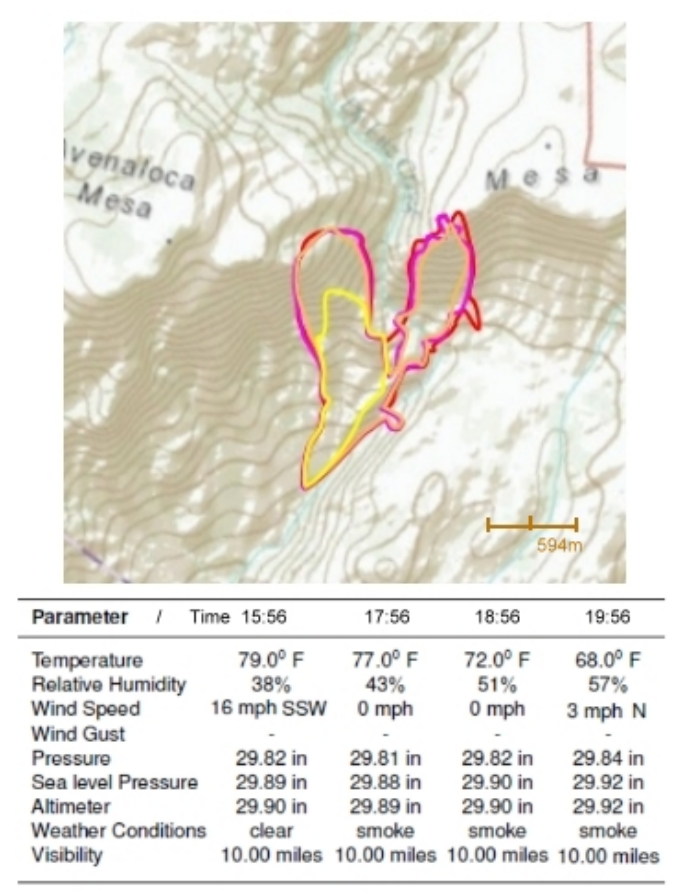

Figure 5.12: Colina fire and weather data - Colina fire and weather conditions. The yellow line represents the fire boundary at 15:36, orange line represents fire boundary at 17:50, pink line represents fire boundary at 18:52 and red line represents fire boundary at 20:06.

We crop the Colina fire area and divide it into a $43 \times 43$ matrix. The burned area is recorded using a global positioning system (GPS) and is captured into GIS data layers. We obtain elevation raster map from the LANDFIRE website [17] to create a contour map. Fig. $5.13 \mathrm{a}$ shows $50 \mathrm{~m}$ interval contours of the Colina area. We enter the vector contours for the majority of the Colina area into GIS at $100 \mathrm{~m}$ intervals and use it to generate a $485 \times 485$ digital elevation model (DEM) of the entire $8430 \times 8430$ meter $^{2}$ Colina area. The highest point in the Colina area is at an elevation of $776 \mathrm{~m}$ higher than the lowest point. To represent vegetation layers we use Anderson's 13 fire behavior fuel models [2] obtained from the LANDFIRE website[17]. 


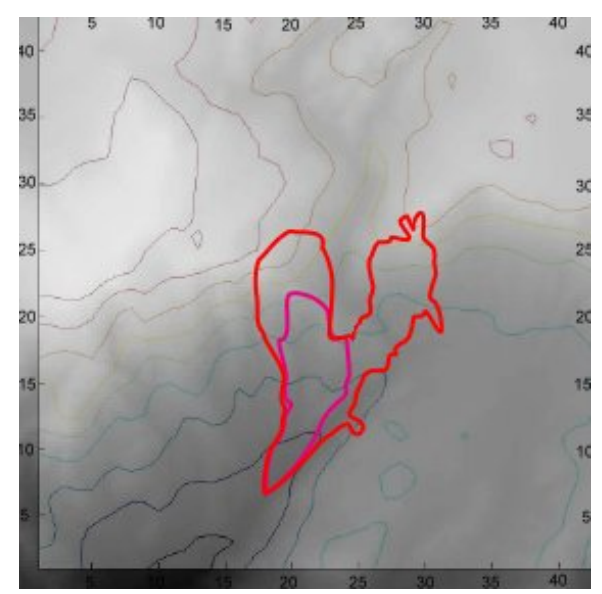

(a) The terrain contour map of the Colina fire area. Elevated land is shown in the lighter shade and lower elevation is shown in the darker shade.

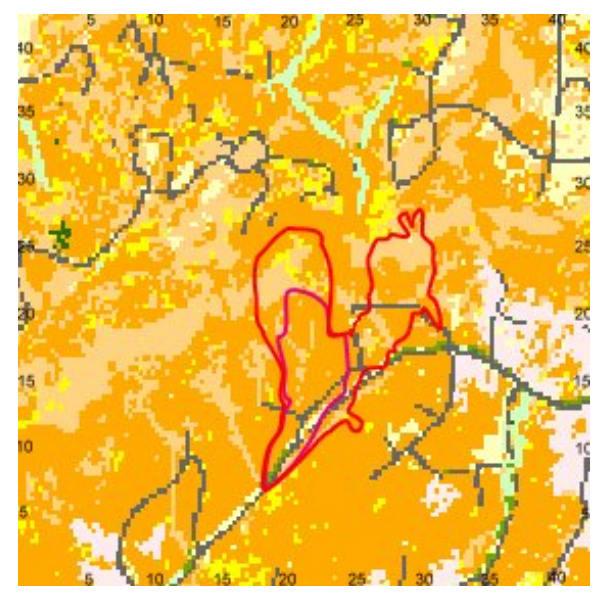

(b) The $13 \mathrm{FBFM}$ vegetation map of the Colina fire area. Most of the vegetation is shrub. The details of color code shown in Fig. 5.1

Figure 5.13: Terrain and vegetation maps of Colina - Contours are transformed from elevation raster maps. The raster data plotted on those two maps are used as data points of $\Phi_{s}$ and $\varepsilon_{0}$ in Eq. (3.9) to calculate the fire spread. We obtain both elevation and vegetation raster maps from LANDFIRE website [17. Red line represents the fire front expansion.

The parameters for fuel are dominated by shrub. The fuel characteristics-fuel load, surface area to volume ratio, fuel bed depth, and moisture of extinction moisture content are shown at Tab. 5.3. The moisture data we assigned to the fuel model is shown at Fig. 5.5 .

\subsubsection{Narrow Band Level Set Method}

From the photo taken for the Colina fire time stamped at 1:35 pm, we observe that the vegetation is rather homogeneous in the area. The wind is SSW at the time of the fire. With this we expect the fire to spread towards the north north east. From the terrain contour map, we see that there is a canyon located to the north which is expected to have a "chimney effect", pushing the fire fast through the canyon. However, the expected chimney effect does not occur. Instead, the fire spreads along both rims 
of the canyon forming a y shape. Likely the canyon is not narrow enough to create a "chimney effect". Fig. 5.14 shows the simulated Colina fire using narrow band level set method.

In order to compare the simulation results to the actual burned area, we color code the simulated burned area red and the actual burned area green (Fig. 5.15). Then we compare the results. In summary, our simulation captures $96 \%$ of the actual burned area; it underestimates $4 \%$ and overestimates $22 \%$.

The overestimated burned area is $22 \%$. This warranted further investigation. At 15:56 PDT there is a SSW wind in the area. We failed to consider, however, that the wind might have changed its speed at the different terrain. Since there is no wind after 17:56 PDT, we modify and reinitialize our simulation after 17:56 PDT with the real fire and restart it at 17:56 PDT with no SSW wind until 20:06 PDT. The revised simulation matches $94 \%$ of the actual burned area, $6 \%$ of the burned area is underestimated and $13 \%$ of the burned is now overestimated. Reinitializing our simulation allows us to reduce the overestimated area by $40 \%$. Tab. 5.2 shows the comparison of the simulation to the real fire before and after the re-initialization.

Table 5.2: Comparison of Colina fire using narrow band level set method Comparison of simulation, simulation after re-initialization, and real fire.

\begin{tabular}{ccc}
\hline Situation & Modification (\%) & Without Modification \\
\hline Estimated Burned Area & $94 \%$ & $96 \%$ \\
Underestimated Burned Area & $6 \%$ & $4 \%$ \\
Overestimated Burned Area & $13 \%$ & $22 \%$ \\
\hline
\end{tabular}

\subsubsection{Fast Marching Method}

We apply the fast marching method to the Colina fire in the same way that we simulate the Troy fire. We calculate the travel time of fire spread to each neighboring cells from a burning cell. The fire then ignites the adjacent cell which processes the shortest travel time around the burning cell; but it ignores the diagonal cells. This limitation causes 


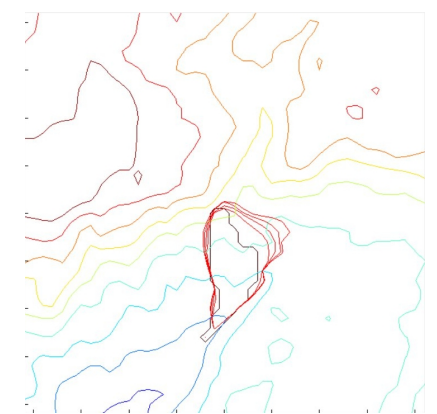

(a) Simu. at 15:30

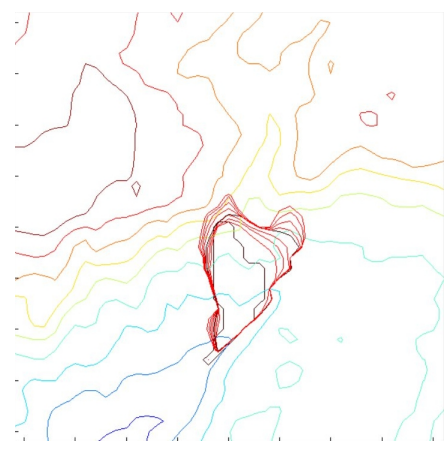

(c) Simu. at 17:00

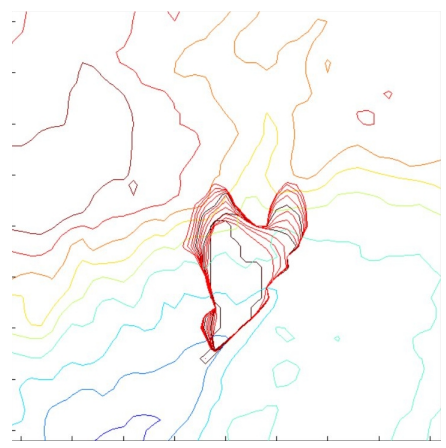

(e) Simu. at 18:30

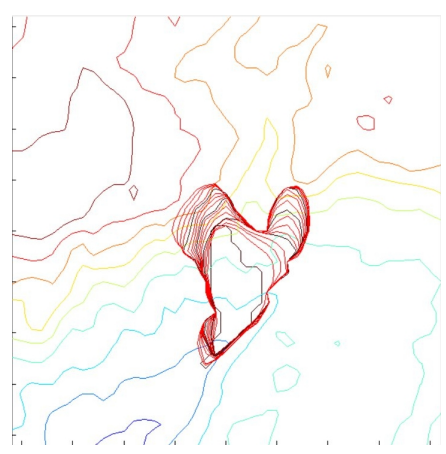

(g) Simu. at 20:00

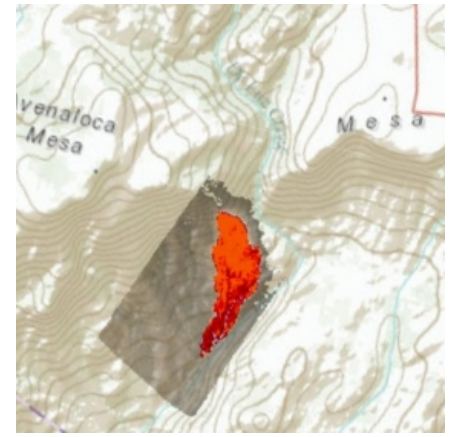

(b) Photo at 15:36

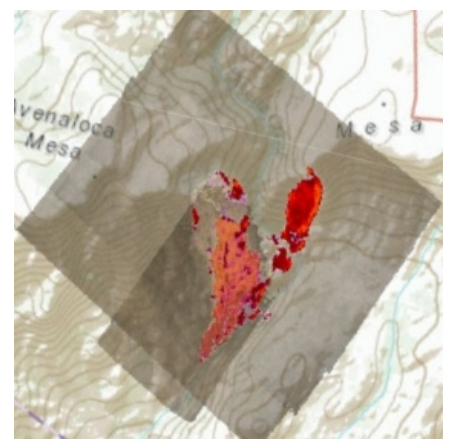

(d) Photo at 17:50

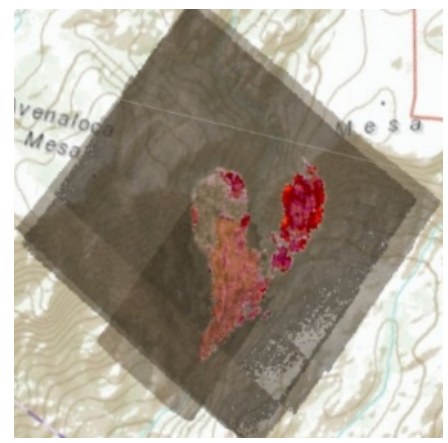

(f) Photo at 18:52

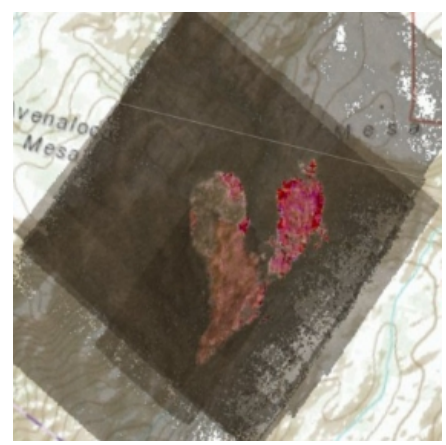

(h) Photo at 20:06

Figure 5.14: Colina fire simulated using narrow band level set method - The simulated image shown on the left side and the actual fire shown on the right. 


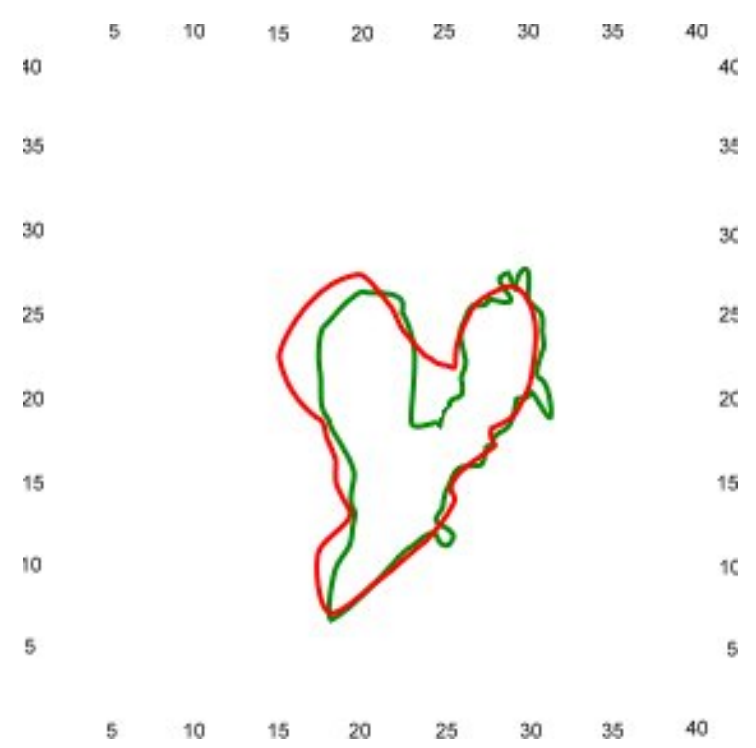

Figure 5.15: Fire simulated using narrow band method compared to actual Colina fire - Boundary comparison after four and half hours fire. The green boundary represents the actual fire front and the red boundary represents the simulated front. $96 \%$ of the actual burned area is burned in the simulation, $4 \%$ is overestimated, and $22 \%$ is underestimated.

the less smooth fire shape and less precise results than those simulated by the narrow band level set method. The result of the Colina fire simulation using fast marching method is shown in Fig. $5.16 \mathrm{a}$

In order to compare the simulation results to the actual burned area, we color code the simulated burned area as red and the actual burned area as green. Then we compare the results from simulation to the actuals. A boundary comparison (Fig. 5.16b) of the actual burned area to the simulated burned area after four and half hours fire reveals $77 \%$ of burned area overlaps. The simulated burn area includes $47 \%$ of land that did not burn at Troy fire (the overestimate) and $23 \%$ of land that burned but was not captured by our simulation (the underestimate). 


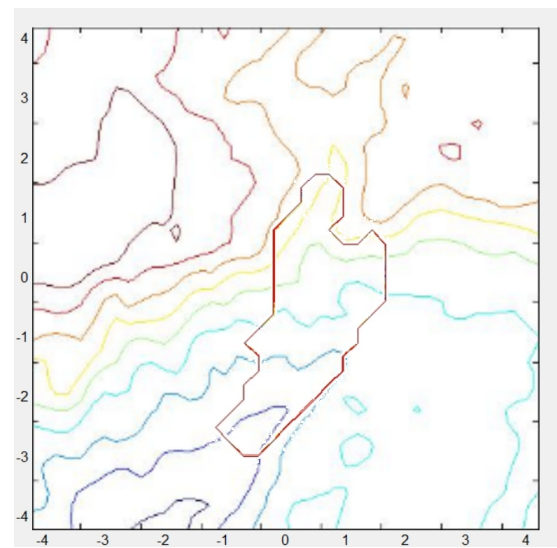

(a) Colina fire simulation using fast marching method

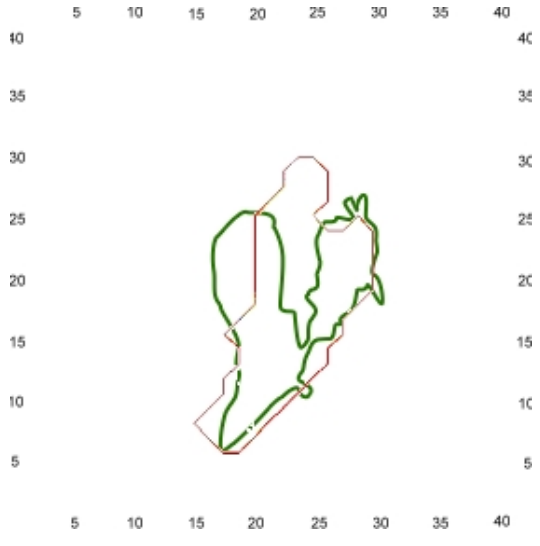

(b) Colina fire simulated using fast marching method compared to the actual Colina fire.

Figure 5.16: Colina fire simulation produced from the fast marching method 5.16a is the result generated from the fast marching method using the modified Rothermel's fire spread rate (Eq. (3.7)). 5.16b shows boundary comparison after four and half hours fire. The green boundary is the actual burned area and the red boundary is the simulated burned area. $77 \%$ of the real burned area overlaps, $47 \%$ is overestimated, and $23 \%$ is underestimated.

\subsection{Numerical Result Analysis}

The comparison of the numerical results for these two implementations is shown at Tab. 5.3. The fast marching method takes longer time than narrow band method because it needs to calculate the travel time for every adjacent cells. The narrow band method needs to calculate only the norm direction based on Huygens' principle and elliptical spread assumption.

The more overlapping area and less underestimated or overestimated area, the better the simulation results are for a simulation model. The narrow band method achieves a better simulation result than the fast marching method in our simulation at domain $[43 \times 43]$. This is because for the fast marching method, we consider only adjacent cells but not diagonal cells as we look for the shortest travel time. This will make the fire spread to have a bias from the diagonal direction. The spread angles limitation in fast marching method also makes the fire shape to be unnaturally angular. Fig. 5.16a shows 
Table 5.3: Case study analysis - The comparisons of simulation results at domain [43 $\times 43]$ using narrow band and fast marching method for Troy fire and Colina fire.

\begin{tabular}{lrrrr}
\hline Simulation Method & \multicolumn{2}{c}{ Simulation Result } & Execution Time(Sec.) \\
\cline { 2 - 3 } & Overlap & Underestimated & Overestimated & \\
\hline Troy Fire & & & & \\
Narrow band method & $92 \%$ & $8 \%$ & $10 \%$ & 11.53 \\
Fast marching method & $89 \%$ & $11 \%$ & $22 \%$ & 37.16 \\
Colina Fire & & & & \\
Narrow band method & $94 \%$ & $6 \%$ & $13 \%$ & 11.33 \\
Fast marching method & $77 \%$ & $23 \%$ & $47 \%$ & 65.29
\end{tabular}

Execution time is the average time of the first three runs.

that the simulation of Colina fire has a bias to the north and produces a large estimation error. For further study, we can consider to find a numerical method to calculate the travel time from a burning cell to its neighboring diagonal cells to improve the accuracy. 
5. CASE STUDIES 


\section{6}

\section{Conclusion}

We implement two level set methods (narrow band and fast marching methods) along with pre-existing fire information data in our fire simulation model which is intended to overcome some of the limitations of the existing fire simulation methods. The great advantage of the level set method is to use higher dimensions to track and simulate the dynamics of moving fire front and provide a solution to a problem which is difficult to be resolved in lower dimensions. The narrow band method is to apply distance function to the moving fire surface while tracking the distance using the fire spread rate as the trigger point to identify the next ignition cell. The fast marching method is to build a travel time table which reflects the time needed for the fire front to cross the grid cell.

The important element of our simulation model is the fire spread rate for the level set evolution. The fire spread rate of Rothermel focuses on the fire heading direction only on a straight line. Therefore, we need to rely on an assumed elliptical fire shape to calculate the other directions of spread rate. Mallet et al. [19] used the level set method to simulate fire spread and produced a good elliptical fire shape. According to Mallet et al. [19], this model still needs to focus on the parameters estimated within the simple model illustrated by his paper to fit different kinds of terrain, fuel bed, etc. Based on the elliptical spread assumption, Mallet's level set model provides a good solution if we consider the wind factor part. We use Mallet's model to replace the wind factor part of Rothermel's fire model to build a modified Rothermel's fire model in our simulation. 


\section{CONCLUSION}

Differentiated from the existing fire spread models which focus on the two dimensions such as the coordinate values on the polygon functions for a vector-based model or the states of each cell in a lattice for raster-based model, our fire simulation model leverages the primary concept of the level set method which introduces the third dimension and solves the difficult crossover problem presented in the existing models. Our research advances the level set method application to a heterogeneous environment by storing pre-existing fire information at the cells. We divide the landscape into identical square cells and define the state of each cell by distance function. We are able to apply the elliptical spread assumption to decide the next ignition point without examining the fire spread to all neighboring cells.

Our contributions include overcoming some of the shortcomings of the simulation model using the vector based method. We made improvements in the following areas:

1. Solved the most difficult crossover issue.

2. Addressed concerns over sharp corners.

3. Stored data at cells which is more suitable for complex environment.

4. Added different vegetation types into consideration for fire spread prediction.

5. Validate fast marching method in the fire spread simulation.

The fire simulation model is verified by two case studies through two fire simulations and meets the expected results. However, there are limitations for the implementations using both the narrow band level set method and the fast marching method. For the level set method, the model needs to re-normalize the distance function to ensure that surface evolution remains stable. It is time consuming and poses the risk of the fire shape becoming distorted. For the fast marching method, more in-depth studies are needed. First, we only consider the adjacent neighboring cells and omit the diagonal neighboring cells. The omissions might cause distortion of the fire shape especially under the condition of the wind. Second, when simulation time is extended, the scale of the graphic will be automatically adjusted to be in alignment with the time axis during Matlab calculation. The automatically rescaled contour map might cause visual distortion and further lead to mis-prediction of the fire spread. Third, the travel time is 
calculated by $1 / F$. An arithmetical operating error will occur for smaller $F$ especially when $\mathrm{F}$ approaches to zero.

With further development, this fire simulation model has the potential to be used for the other spread simulation researches such as predicting smog pollution, oil spill and volcano ashes spread. The concept of applying the projection to transform a discrete process to continuous process can be developed to improve the quality of image from the methods based on discrete procession. This can lead to a marker-less method in simulation technique. 
6. CONCLUSION 


\section{Bibliography}

[1] D.H. Anderson, E.A. Catchpole, N.J. DE Mestre, and T. Parkes. Modelling the spread of grass fires. $J$. Austral. Math. Soc. (Series B) 23, 451-466, 1982. 14

[2] Hal E. Anderson. Aids to determining fuel models for fire behavior. General Technical Report INT-122, United States Department of Agricuture Forest Service Intermountain Forest and Range Experiment Station, Ogden, UT 84401, April 1982. xiii, 42, 43, 63, 66, 73

[3] ArcGIS. The ArcGIS Webside, 2011. URL http://www.esri.com/ software/arcgis/index.html. 63

[4] National Fire Protection Association. The USFA Webside /Statistics, 2011. URL http: //www.usfa.fema.gov/statistics/ estimates/wildfire.shtm. 1

[5] Stephen G Berjak and John W Hearne. An improved cellar automaton model for simulating fire in a spatially heterogeneous savanna system. Ecological Modelling, 148:133-151, 2002. 21

[6] G. M Byram. Combustion of forest fuels. In: Forest Fire - Control and Use, pages 61-89. McGraw Hill, 1959. 44, 46

[7] E. W. Dijkstra. A note on two problems in connection with graphs. Numerische Mathematic, 1:269-271, 1959. 32

[8] F.E.Fendell and M.F. Wolff. Forest firesBehavior and ecological effects, chapter 6: Wind-Aided Fire Spread, pages 171223. Academic Press, 2001. 14

[9] Mark A. Finney. Farsite:fire area simulator-model development and evaluation. Research Paper RMRS-RP-4 Revised, United States Department of Agricuture Forest Service Rocky Mountain Research Station, March 1998. 3 . 13, 16, 44, 46.

[10] WL Fons. Analysis of fire spread in light forest fuels. Journal of Agricultural Research, 72(3):93-121, 1946. 7

[11] W. H. Frandsen. Fire spread through porous fuels from the conservation of energy. Combust, Flame, 16:9-16, 1971. 10

[12] HT Gisborne. The objectives of forest fire-weather research. Journal of Forestry, 25(4):452-456, 1927. 7

[13] HT Gisborne. The complicated controls of fire behavior. Journal of Forestry, 27(3):311-312, 1929. 7

[14] LF Hawley. Theoretical considerations regarding factors which influence forest fires. Mournal of Forestry, 24(7):756763, 1926. 7

[15] French Ian. Visualisation techniques for the computer simulation of bushfires in two dimensions. Master's thesis, Department of Computer Science, University College, University of New South Wales, 1992. 13, 17 
[16] I. Knight and J. Coleman. A fire perimeter expansion algorithm-based on huygens wavelet propagation. International Journal for Wildland Fire, 3(2):73-84, 1993. 13

[17] LANDFIRE. The LANDFIRE Webside /Data Products, 2011. URL http:// www.landfire.gov/, 59, 63, 66, 67, 73, 74

[18] R.H. Luke and A.G. McArthur. Bushfires in Australia. Canberra: Australian Government Publishing Service, 1978. 44,46

[19] V. Mallet, D.E. Keyes, and F.E. Fendell. Modeling wildland fire propagation with level set methods. Computers and Mathematics with Applications, 2009. 15, 16. 19, 20, 21, 23, 29, 81

[20] Matlab. The Matlab Webside. URL http://www.mathworks.com/ products/matlab/ 37

[21] I. R. Noble, G. A. V. Bary, and A. M. Gill. Mcarthur's fire-danger meters expressed as equations. Aust. J. Ecology, 5:201-203, 1980. 9

[22] S. Osher and J.A. Sethian. Fronts propagating with curvature dependent speed: Algorithms based on hamilton-jacobi formulations. Computational Physics, 1988. 4, 13, 19, 22, 60

[23] E. Paster, L. Zarate, E. Planas, and J. Arnaldos. Mathematical models and calculation systems for the study of wildland fire behaviour. Progress in Energy and Combustion Science, 29(2):139-153, 2003. doi: http://www.floresta.ufpr.br/ firelab/artigos/artigo221.pdf. 1, 8
[24] Danping Peng, Barry Merriman, Stanley Osher, Hongkai Zhao, and Myungjoo Kang. A pde-based fast local level set method. Journal of Computational Physics, 155:410-438, 1999. 26

[25] G.D. Richards. An elliptical growth model of forest fire fronts and its numerical solution. International Journal for Numerical Methods in Engineering, 30: 1163-1179, 1990. 13

[26] GD. Richards and R.W. Bryce. A computer algorithm for simulating the spread of wildland fire perimeters for heterogeneous fuel and meteorological conditions. International Journal for Wildland Fire, 5(2):73-80, 1995. 13

[27] James Rickett and Sergey Fomel. A second-order fast marching eikonal solver. SEP Report, 100:287-292, 1999. 35

[28] R.C. Rothermel. A mathematical model for predicting fire spread in wildland fuels. Research Paper RMRS-GTR-153, United States Department of Agricuture Forest Service Rocky Mountain Research Station, 1972. 4, 7, 8, 10,12

[29] Joe H. Scott and Robert E. Burgan. Standard fire behavior fuel models:a comprehensive set for use with rothermel's surface fire spread model. General Technical Report RMRS-GTR-153, United States Department of Agricuture Forest Service Rocky Mountain Research Station, June 2005. xiii, 42

[30] J. A. Sethian. Level Set Method and Fast Marching Methods: Evolving Interfaces 
in Computational Geometry, Fluid Mechanics, Computer Vision, and Materials Science. Cambridge University Press, 1999. 4. 61

[31] J. A. Sethian. Fast marching methods. SIAM Review, 41:199-235, 1999. URL http://www.jstor.org/stable/ 2653069, 30, 32, 33, 34

[32] Pacific Southwest Research Station. The USDA Webside Fire Science/troy, February 2007. URL http://www.fireimaging.com/fires/ 2002/troy/index.htm1. 64, 65

[33] A.L. Sullivan. Wildland surface fire spread modelling, 1990-2007. 3: Simulation and mathematical analogue models. International Journal of Wildland Fire, 18(4):387-403, 2009. doi: http: //dx.doi.org/10.1071/WF06144. 1. 13 . 17
[34] Andrew L. Sullivan. Wildland surface fire spread modelling, 1990-2007. 1:physical and quasi-physical models. International Journal of Wildland Fire, 18(4):349-368, 2009. doi: http://www. publish.csiro.au/?paper $=$ WF06143. 1,8

[35] PH Thomas. Rates of spread of some wind-driven fires. Journal of Forestry, 44:155-175, $1971 . \quad$ doi: http://forestry.oxfordjournals.org/ content/44/2/155.abstract. 9

[36] J.N. Tsitsiklis. Efficient algorithms for globally optimal trajectories. IEEE Transactions on Automatic Control, 40: 1528-1538, 1995. 34

[37] G. Wallace. A numercial fire simulation model. International Journal for Wildland Fire, 3(2):111-116, 1993. 13 